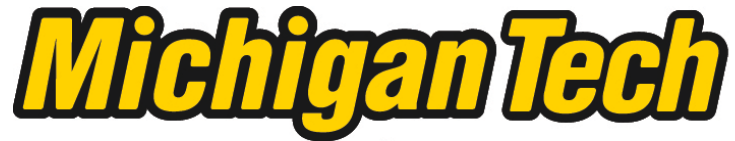 \\ Michigan Technological University Create the Future Digital Commons @ Michigan Tech
}

2015

\section{AUTOMATED SCOUR DETECTION ARRAYS USING BIO-INSPIRED MAGNETOSTRICTIVE FLOW SENSORS}

Baibhav Rajbhandari

Michigan Technological University

Follow this and additional works at: https://digitalcommons.mtu.edu/etds

Part of the Engineering Commons, and the Sustainability Commons Copyright 2015 Baibhav Rajbhandari

\section{Recommended Citation}

Rajbhandari, Baibhav, "AUTOMATED SCOUR DETECTION ARRAYS USING BIO-INSPIRED

MAGNETOSTRICTIVE FLOW SENSORS", Master's Thesis, Michigan Technological University, 2015.

https://doi.org/10.37099/mtu.dc.etds/959

Follow this and additional works at: https://digitalcommons.mtu.edu/etds

Part of the Engineering Commons, and the Sustainability Commons 


\title{
AUTOMATED SCOUR DETECTION ARRAYS USING BIO- INSPIRED MAGNETOSTRICTIVE FLOW SENSORS
}

\author{
By
}

Baibhav Rajbhandari

\begin{abstract}
A THESIS
Submitted in partial fulfillment of the requirements for the degree of MASTER OF SCIENCE

In Civil Engineering
\end{abstract}

MICHIGAN TECHNOLOGICAL UNIVERSITY

2015

(C) 2015 Baibhav Rajbhandari 
This thesis has been approved in partial fulfillment of the requirements for the Degree of MASTER OF SCIENCE in Civil Engineering.

\section{Department of Civil and Environmental Engineering}

Thesis Advisor: $\quad$ Dr. Raymond Andrew Swartz

Committee Member: $\quad$ Dr. Brian Barkdoll

Committee Member: Dr. Timothy Havens

Department Chair: $\quad$ Dr. David Hand 


\section{Table of Contents}

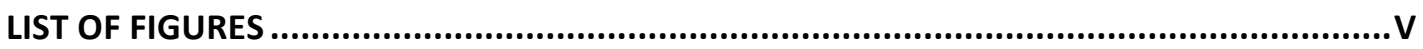

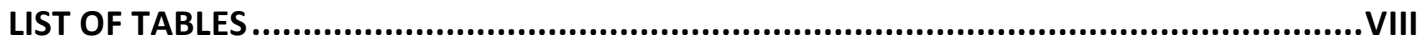

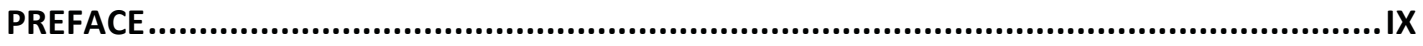

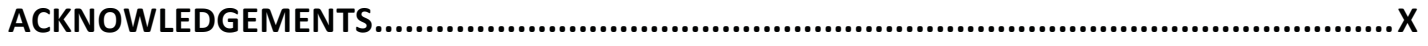

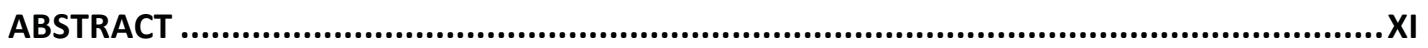

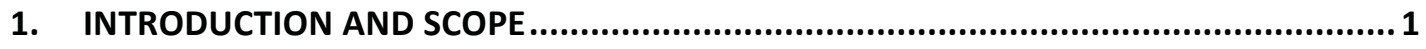

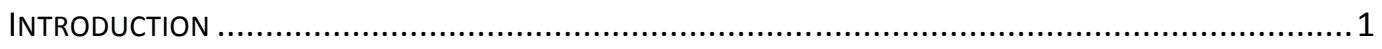

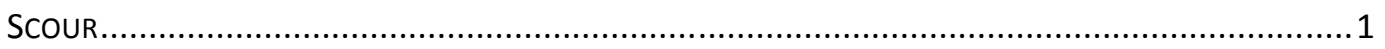

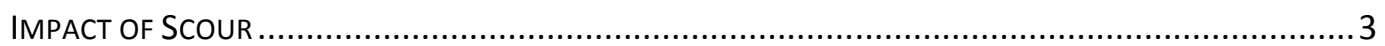

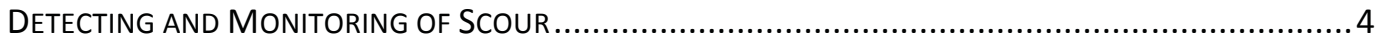

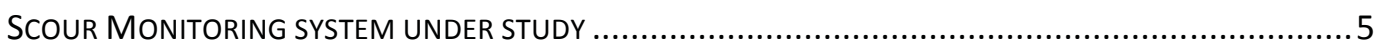

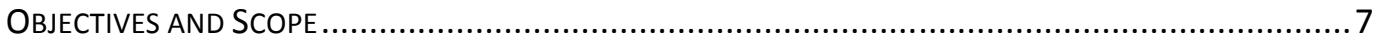

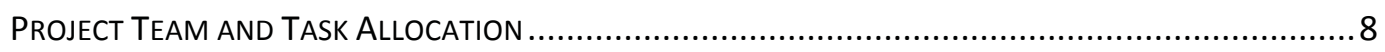

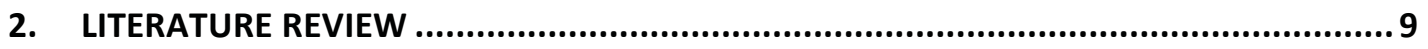

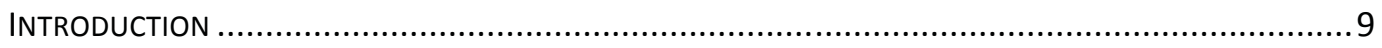

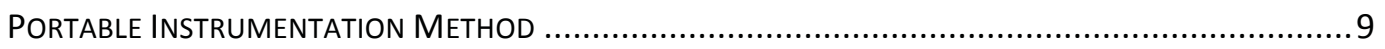

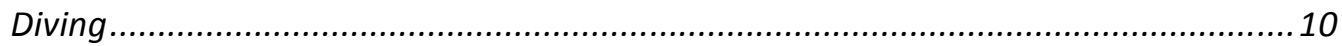

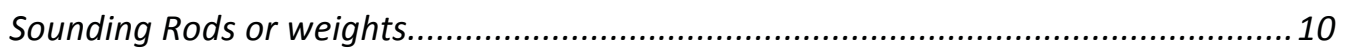

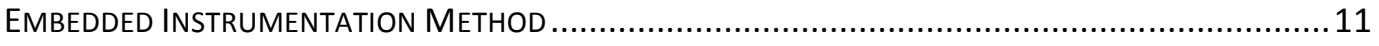

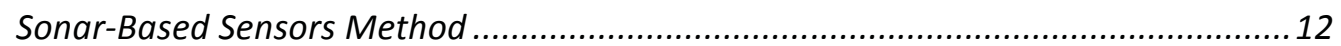

Time Domain Reflectometer (TDR) Method........................................................... 12

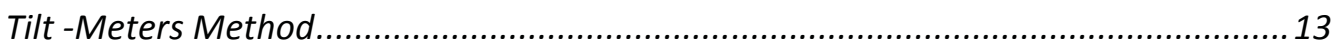

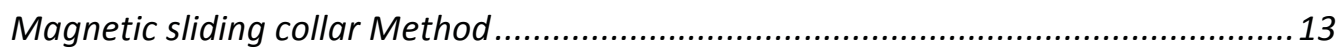

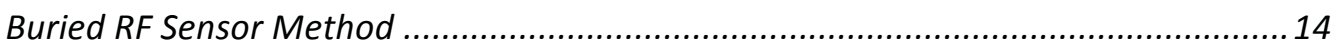

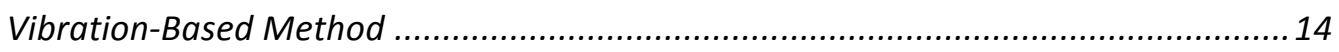

Acoustic Doppler Current Profiler Method ............................................................... 15

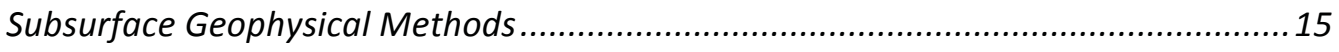

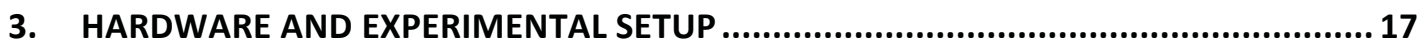

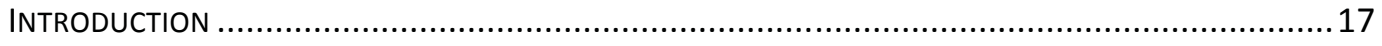

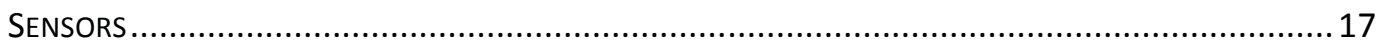

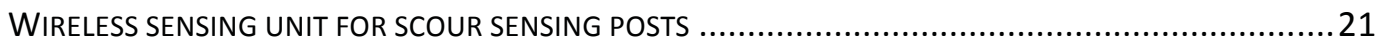

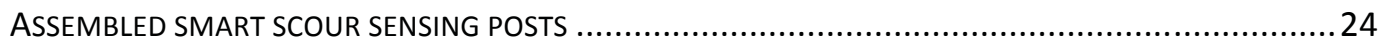

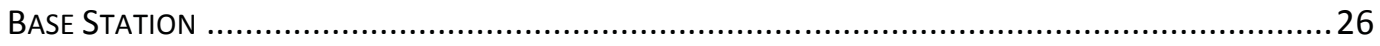

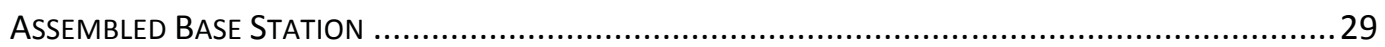




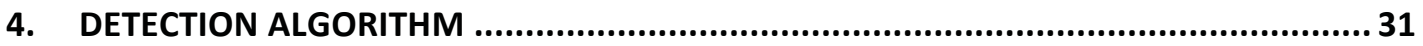

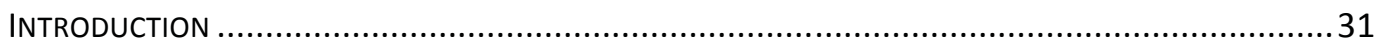

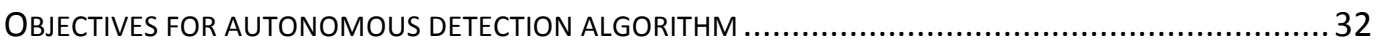

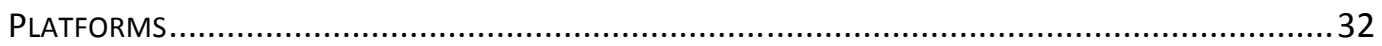

OPERATION

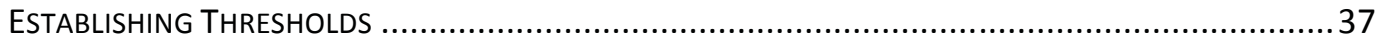

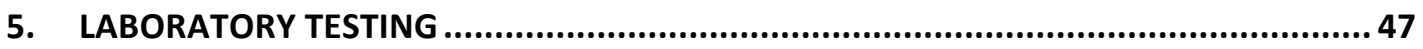

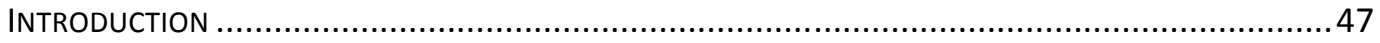

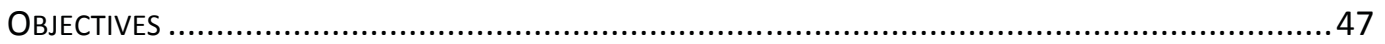

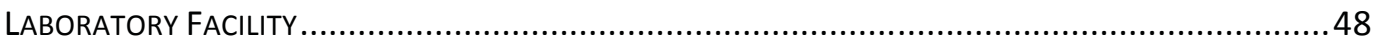

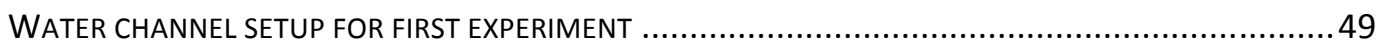

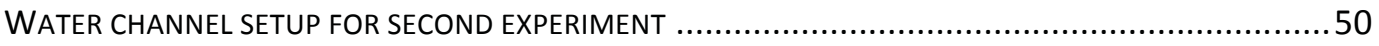

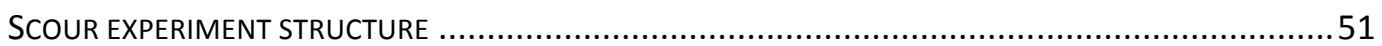

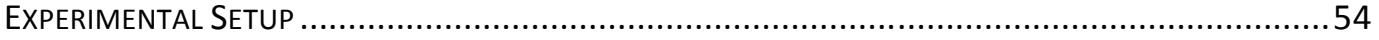

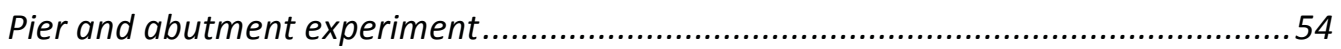

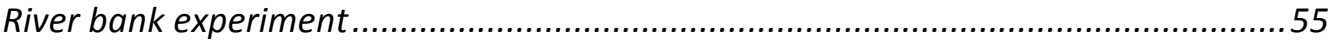

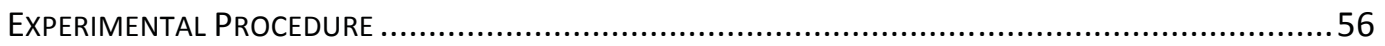

Scour Depth Plot Pier and abutment experiment .....................................................5 58

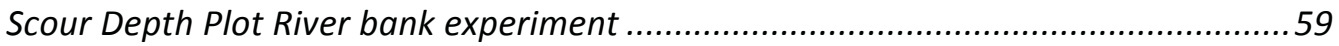

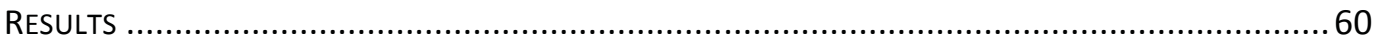

DATA COLLECTION ANALYSIS OF LABORATORY EXPERIMENT: MODEL PIER AND ABUTMENT ..................61

DATA COLLECTION ANALYSIS OF LABORATORY EXPERIMENT: MODEL POST ......................................66

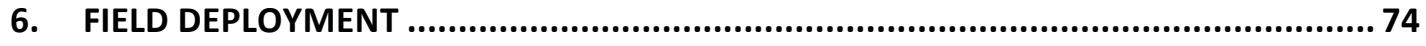

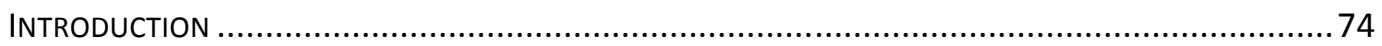

SELECTED BRIDGE FOR FIELD IMPLEMENTATION AND EVALUATION.......................................... 74

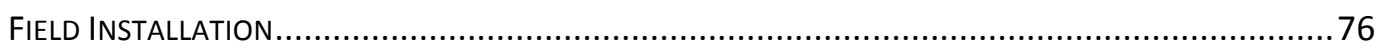

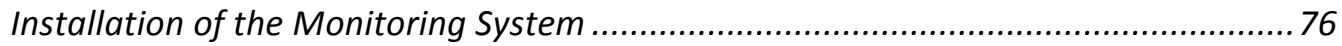

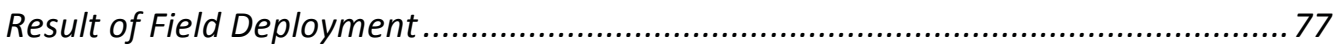

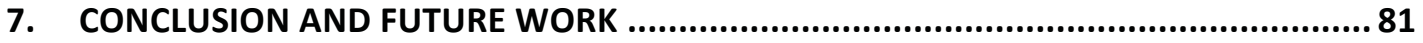

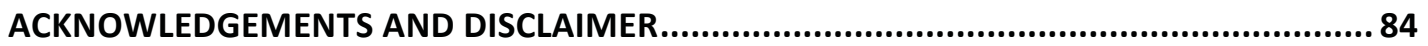

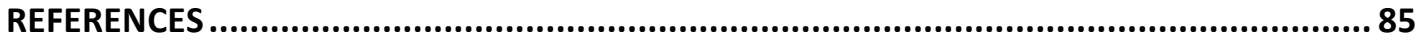

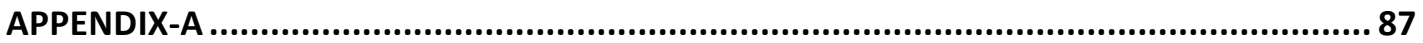




\section{List of Figures}

Figure 1. Scour ..................................................................................................... 1

Figure 2. Cause of Bridge Failure (1966-2005) (Data Source: New York State DOT

and Texas A\&M University) ........................................................................... 3

Figure 3. Schoharie Creek Bridge Collapse (Image Source

http://water.usgs.gov/wid/images/NY.figure.id.3.gif, See Appendix-A for

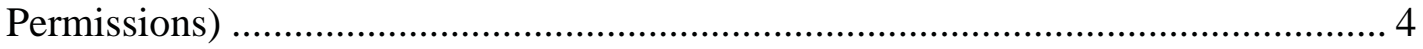

Figure 4. (a) \& (b) Working of Proposed System ................................................. 6

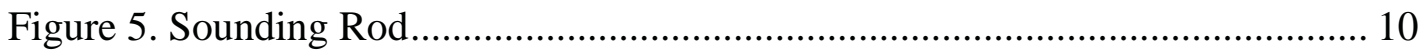

Figure 6. Sonar Based Sensors ............................................................................... 12

Figure 7. Time Domain Reflectometer.................................................................... 12

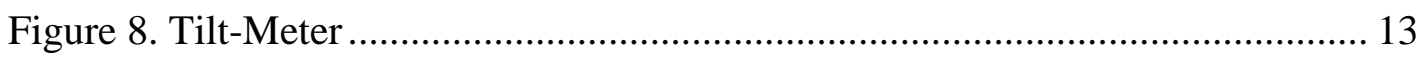

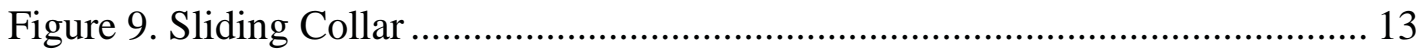

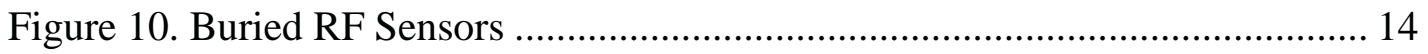

Figure 11. Acoustic Doppler ............................................................................. 15

Figure 12. Marine Animals with Whiskers (Image Source www.flickr.com, See

Appendix-A for Permissions ................................................................................. 17

Figure 13. Bio-inspired Magnetostrictive Whisker Sensor .................................... 18

Figure 14. Working Principle of Magnetostrictive Sensor (Image by Author)........ 19

Figure 15. Airfoil-type Sensor (Image by Author) .............................................. 20

Figure 16. Seaweed-type Sensor (Image by Author) ......................................... 20

Figure 17. Narada WSU (Image by Author) .......................................................... 21

Figure 18. Antenna and Radio Daughter Board (Image by Author) ....................... 21

Figure 19. Post Electronics Packaged in Polycase Enclosure (Image by Author) ... 25

Figure 20. Packaged Smart Scour-sensing Post (Image by Author) ...................... 25

Figure 21. Single Board Computer for Base Station (PPM-LX800-G) (Image by

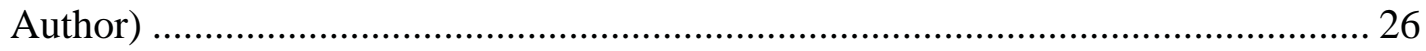

Figure 22. UML 290 (Image by Author) ............................................................ 27

Figure 23. Narada Base Station (Image by Author) ............................................... 28

Figure 24. Base Station Electronics in Protective Enclosure (Image by Author) .... 30

Figure 25. Assembled Based Station Installed in the Field (Image by Author) ....... 30

Figure 26. Basic Algorithm Steps .................................................................. 34

Figure 27. Low Flow Condition Time Domain and Frequency Domain Response for

Buried and Unburied sensors........................................................................ 38

Figure 28, High Flow Condition Time Domain and Frequency Domain Response for Buried and Unburied Sensors ........................................................................... 38 
Figure 29. Histogram of Standard Deviation for Airfoil Type Sensors at Different States.

Figure 30. Histogram of Magnitude of FFT for Airfoil Type Sensors at Different

States

Figure 31. Histogram of Standard Deviation for Whisker Type Sensors at Different

States 40

Figure 32. Histogram of Magnitude of FFT for Whisker Type Sensors at Different

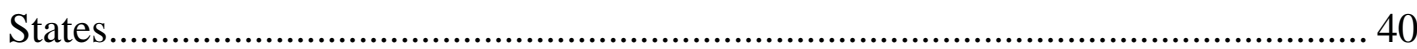

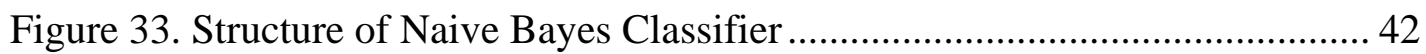

Figure 34. Bayes Classifier for Airfoil Sensor Using Standard Deviation................ 43

Figure 35. Bayes classifier for Airfoil Sensor Using Magnitude of FFT ................. 44

Figure 36. Decision File (Image Source Microsoft Excel, See Appendix-A for

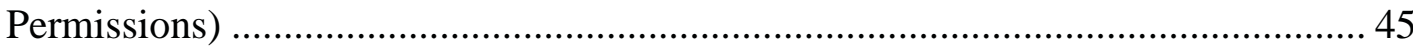

Figure 37. Flow Chart Depicting Narada WSU Algorithm in Scour Sensing Post.. 46

Figure 38. Flume Structure (Image by Author) .................................................... 49

Figure 39. Flume Setup for First Experiment (Image by Author) ............................. 50

Figure 40. Flume Setup for Second Experiment (Image by Author) ........................ 51

Figure 41. Pier and Abutment (Image by Author) …………................................ 52

Figure 42. Sample Airfoil Posts (Image by Author) ................................................. 53

Figure 43. Flume Setup for Pier and Abutment Experiment (Image by Author)..... 55

Figure 44. River Bank Experiment (Image by Author)........................................... 56

Figure 45. Scour Hole Developed (Image by Author) ………................................. 57

Figure 46. Scour Depth Plot Pier and abutment experiment .................................... 58

Figure 47. Scour Depth Plot River bank experiment ……….................................. 59

Figure 48. Time Domain Plot of Buried Sensor (Static State) ................................... 60

Figure 49. Time Domain Plot of Unburied Senor (Dynamic State).......................... 61

Figure 50. Time Domain Plot at Low Water Velocity for WSU 77 ......................... 62

Figure 51. Time Domain Plot at Medium Water Velocity for WSU 66 ................... 63

Figure 52. Time Domain Plot at Medium Water Velocity for WSU 77 ................... 64

Figure 53. Time Domain Plot at Medium Water Velocity for WSU 66 ................... 64

Figure 54. Time Domain Plot at Medium High Velocity for WSU 77 ..................... 65

Figure 55. Time Domain Plot at Medium Water Velocity for WSU 66 ................... 66

Figure 56. Time Domain Plot at Low Water Velocity for WSU 7 ............................ 67

Figure 57. Time Domain Plot at Low Water Velocity for WSU 53........................ 68

Figure 58. Time Domain Plot at Medium Water Velocity for WSU 7 ................... 69

Figure 59. Time Domain Plot at Medium Water Velocity for WSU 53 .................. 70

Figure 60. Time Domain Plot at Medium Water Velocity for WSU 7 .................... 71

Figure 61. Time Domain Plot at Medium Water Velocity for WSU 53 .................. 72 
Figure 62. Success Rates of Sensors

Figure 63. Pilgrim River Bridge (Image Source https://www.google.com/maps,See Appendix-A for Permissions)

Figure 64. Sturgeon River Bridge (Image Source https://www.google.com/maps,

See Appendix-A for Permissions).

Figure 65. (a) and (b) Auger (c) Procedure for Drilling of Hole (Image by Author) 78

Figure 66. (a) and (b) Manual Installation of Post, (b) and (c) Installed post (Image

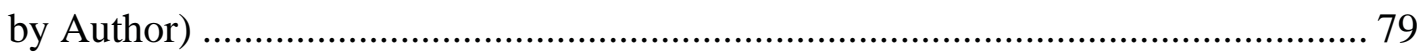

Figure 67. (a) Solar Panel and (b) Installation of Base Station (Image by Author) 80 


\section{List of Tables}

Table 1. Technical Specification of Narada WSU [21] ..................................... 23

Table 2. Technical Specifications of SBC (WinSystems) [22] .............................. 27

Table 3. Technical Specifications UML 290 [23] ................................................ 28

Table 4. Narada Base Station Technical Specifications: (Civionics) [21] ............... 29

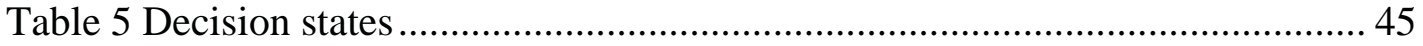




\section{Preface}

This Dissertation is submitted for the Degree of Master of Science in Civil Engineering at Michigan Technological University. This preface serves as an explanation of my role in the work that comprises each of the chapters that make up the body of this thesis.

The studies in this project have been performed by Michigan Technological University and University of Maryland. Other partners involved in the project were Michigan Department of Transportation, Maryland Department of Transportation, and Civionics, LLC.

Part of this work contains previously published material and material submitted for journal review. A paper was published at the ASME Conference Proceedings (Author R. Andrew Swartz, Baibhav Rajbhandari and Benjamin D. Winter Paper No. SMASIS2014-7694, pp. V001T05A013) from the work of this project. A Project website was also created for the project.

In this study I was responsible for developing a working algorithm and establishing thresholds for scour monitoring. I worked on laboratory data collection, building of models, and data analysis of resulting laboratory data. Jennie Tyrrell and Megan MacNeill helped me with the laboratory testing of the proposed system. I was also responsible for assembling the smart scour sensing posts and the base station that was used in the field installation. I was assisted by Benjamin Winter during this work. I was also actively involved during the field deployment of the developed system. 


\section{Acknowledgements}

First and foremost I would like to offer my sincerest gratitude and thanks to my advisor, Dr. R. Andrew Swartz, you have been a tremendous mentor for me. Your guidance has helped me in all times of research and writing of this thesis. I could not have imagined having a better advisor and mentor for my MS study I would like to thank you for encouraging my research and for allowing me to grow.

I would also like to thank Dr. Brian Barkdoll for who has helped me a lot with the laboratory testing and guidance throughout my research. I would like to thank Benjamin Winters, who as a good friend was always willing to help and give his best suggestions. It would have been a lonely lab without him.

I would also like to thank Jennie Tyrrell and Megan MacNeill who helped me during the laboratory testing. They were always willing to help which made my life easier.

I would also like to thank my family and friends who have always been there when I needed them. 


\section{Abstract}

Scour is the most common cause of catastrophic bridge failures worldwide. Approximately over $60 \%$ of bridge failures reported in the United States from 1966 to 2005 are scour related. To ensure the continued safe operation of bridges, monitoring bridge scour is of paramount importance. Most monitoring regimes that are widely used are based on expensive underwater instrumentation. This research focuses on scour detection using automated remote flow detection arrays based on bio-inspired flow sensors. This study employs an array of bio inspired flow sensors that are inexpensive and robust versions of buried-rod scour sensor arrays, coupled with low-power wireless sensor network utilizing civil-engineering domain wireless sensing units to detect scour around bridge piers and abutments. Sensors within the network that report dynamic flow signals are considered to be waterborne or located above the sediment and sensors reporting static signals are characterized as buried or as being located in sediment. The a priori information of sensor depth will help to establish the sediment level in real time. An automated data interrogation system collects data, processes the raw sensor data using in-network data interrogation methods, then and communicates the results to the on-site base station. The relative directness of this data interrogation adds to the robustness of the system. The main purpose of the scour detection system is to provide remote scour information to bridge owners in a format that is easy to comprehend as an aid in decision making. In this project, only processed results, not raw data, are transmitted to the user. The system under study utilizes a cellular data link to relay simplified data to the bridge owner to aid in decision making. A robust program of validation has been conducted to define the limits of the approach in the laboratory and the results of the laboratory validation experiments have been presented in this thesis. This thesis also illustrates the ongoing initial field installation of scour monitoring system on local bridges with some scour concern. 


\section{Introduction and Scope}

\section{Introduction}

Scour is a process of channel erosion around structural supports that can result in development of scour holes around the bridge structures that significantly reduce the foundation stiffness which may lead to abrupt collapse of the structure. It is not easy to prevent scour [1]. However, scour can be monitored over time and, if required, remediation measures can be implemented to avert the collapse of the structure or prevent the public from using it.

This chapter presents an introduction to scour and details its impact on civil structures such as bridges, abutments, and ridge piers. It also introduces the difficulties that are faced during the monitoring and measurement of scour, and chapter briefly describes the conventional methods of scour detection that are commonly used (a topic presented in more detail in Chapter 2). This chapter also gives an introduction to scour detection using automated remote flow detection arrays based on bio-inspired magnetostrictive flow sensors that this research focuses on.

\section{Scour}

"Scour is the result of the erosive action of flowing water, excavating and carrying away material from the bed and banks of streams and from around the piers and abutments of bridges" [2]. As such, scour can precipitate bridge

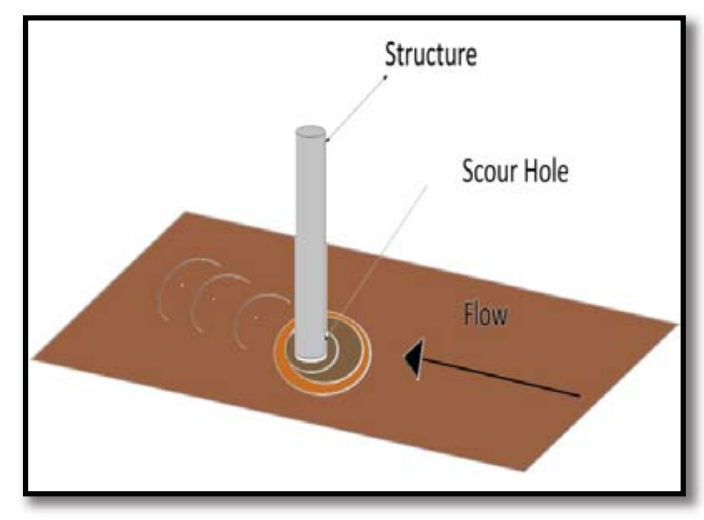

Figure 1. Scour collapse when the depth of scour hole formed near the foundations make them unstable. Scour can be categorized into three forms according to its occurrence as 
general scour, contraction scour, and local scour [3]. The depth of scour around a bridge structure is due to a combined effect of general, contraction and local scour. Scour that is caused as a result of lowering of the stream bed elevation over a short period of time or due increase in the elevation of bed is known as general scour. Change in elevation of the stream bed may occur naturally due to slow transport of sediment which results in lowering of elevation or due to slope failure in the upstream causing deposition of soil downstream, increasing the elevation of the stream bed. General scour can also occur due to simulated causes such as artificial straightening of river. Contraction scour occurs due to the increase in the water velocity which is caused by the decrease in cross-sectional area of the channel. The decrease in cross-sectional area is generally caused by construction of structures such as bridge piers and abutments or approach embankments. The increases in water velocity amplify the erosive action of water resulting in increased scour at the locality of a bridge [3]. Local scour takes place around the vicinity of the structure when a structure obstructs the flow of water or causes a change in pattern of the flow pattern. The change in flow pattern disrupts the equilibrium between actual sediment load and the capacity of the flow to carry sediment resulting in scour to restore equilibrium [4].

Civil structures sit on foundations which are, ideally, constant over time and their presence prevents collapse. Any course of action or natural process that undermines the foundations or the soil around them also causes damage to the structure. Bridge structures as well as embankments near roadways are mainly susceptible to this kind of attack when high-velocity water flows and transfer sediment away from the bridge foundation. Bridge substructures consist of piers, abutments, and foundations, which are vital elements for safe operation of bridges. A bridge is considered scour critical when its foundations have been determined to be unstable for the calculated or observed scour condition [5]. Such bridges require extra attention from bridge owners. 


\section{Impact of Scour}

Scour is considered one of the major causes of bridge failures in the United States. More than 20,000 bridges in the United States are considered scour critical [6]. More bridge failures are due to scour than to all other factors combined. Figure 2 shows the contribution of different causes of bridge failure during 1996 to 2005 in the United States. It can be clearly seen that scour resulted in the highest number of bridge failure during this time period compared to other contributing causes. The 1987 catastrophic collapse of the Schoharie Creek Bridge in the state of New York is a prominent example of bridge failure due to scour (Figure 3). The chief cause of the Schoharie Creek Bridge failure was reported to be scour under a plain concrete pier footing [7]. This incident showed the devastating nature of scour damage and signified the need and importance of proper scour monitoring.

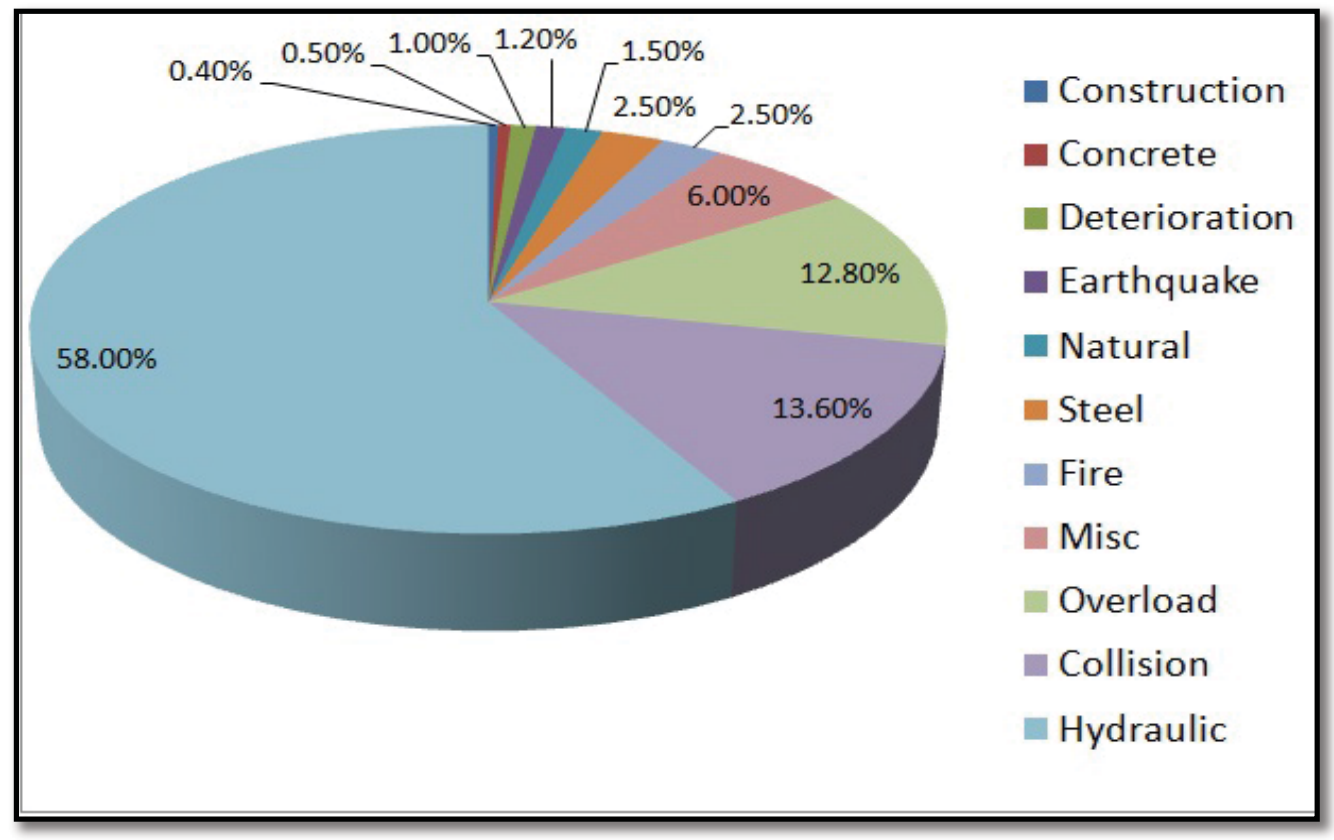

Figure 2. Cause of Bridge Failure (1966-2005) (Data Source: New York State DOT and Texas A\&M University) 


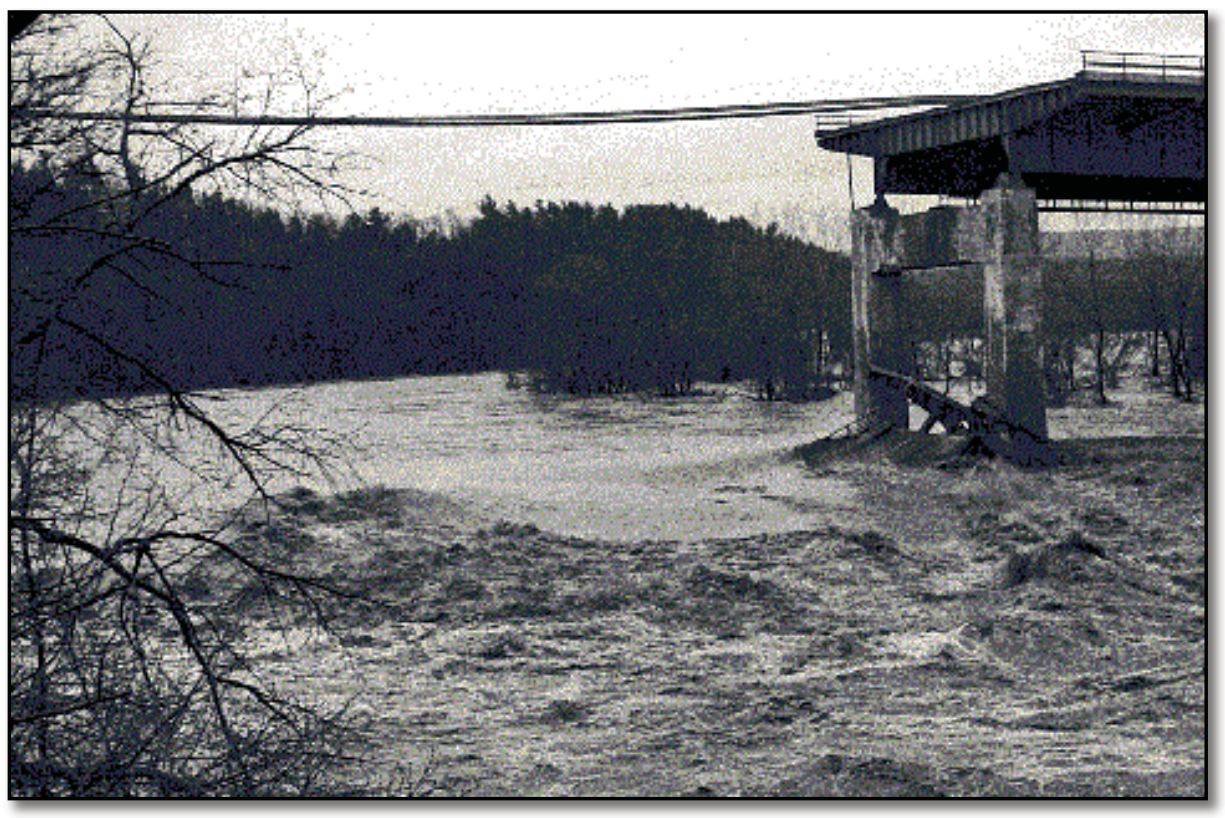

Figure 3. Schoharie Creek Bridge Collapse (Image Source http://water.usgs.gov/wid/images/NY.figure.id.3.gif, See Appendix-A for Permissions)

\section{Detecting and Monitoring of Scour}

Monitoring scour is a very useful countermeasure to deal with bridge failure caused by scour, as potential scour problems can be identified early and required course of action can be taken. However, since scour occurs under the channel flow and usually cannot be visually detected, it is not easily discernible [8]. The flow of water may deposit or erode the sediments in the river bed over time making it more difficult to detect scour.

Scour monitoring allows for action to be taken before the potential failure of a bridge which may endanger the safety of the public. Nearly all of the methods that are currently used to measure scour suffer from some drawback in that they are either expensive, are only capable of measuring maximum scour (thus they do not monitor scour continuously), or do not work in difficult but common water 
conditions, such as turbulent, sediment-filled, or icy water conditions. The most commonly used methods to measure scour are visual inspection, sonar technology, sounding rod, and diving method, along with a few embedded instrumentation methods such as magnetic sliding collar and Tilt sensors. The relative merits of these approaches are presented in Chapter 2 of this thesis.

\section{Scour Monitoring system under study}

The approach of scour monitoring under study is automated, remotely operated embedded sensor networks that have the ability to measure and record scour conditions on a continuous basis. The scour sensor network in study utilizes an array of bio-inspired flow sensors along with low power, inexpensive sensor network. A diagram of the system under study is shown in Figure 4.

The scour monitoring system under study is aimed at being inexpensive, easy to

install, and low maintenance. The system is being designed to be robust, which will make it difficult to damage and suitable for different working conditions such as turbulent water flow and conditions with large amount of debris and sediment. The robust nature of the transducers adds to the robustness of the system. The system will also be remotely operated and automated, making it easier to monitor scour on different conditions and also eliminating the requirement of having experienced personnel at site. 


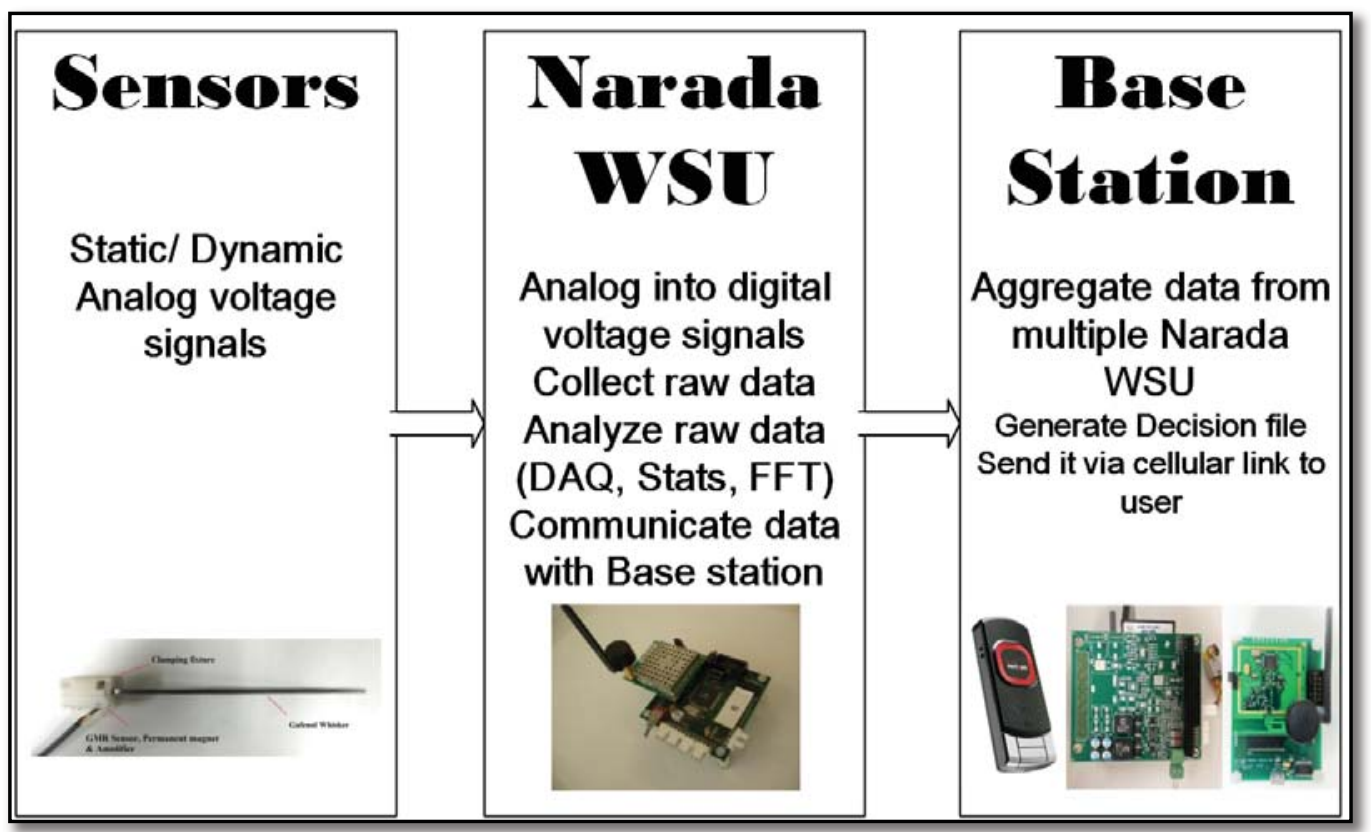

(a)
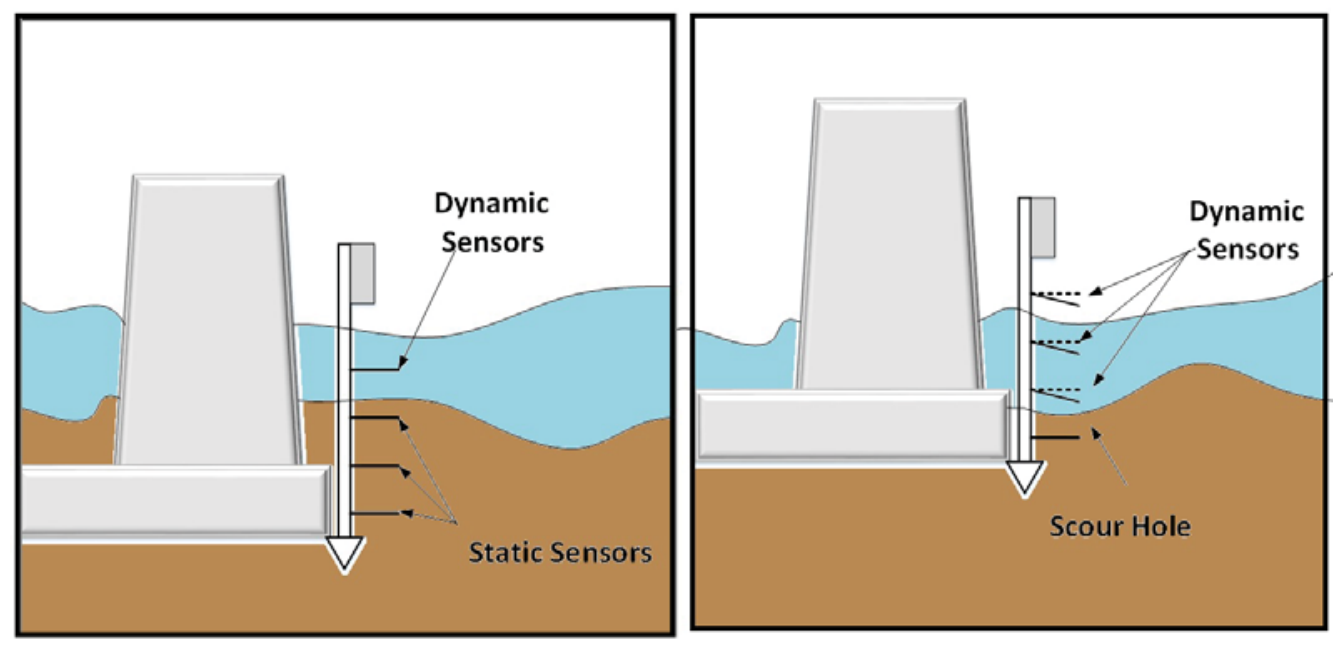

(b)

Figure 4. (a) \& (b) Working of Proposed System 


\section{Objectives and Scope}

The main objectives of the research are:

1. Laboratory validation of the automated scour detection and monitoring system that is based on bio-inspired flow sensing sensors.

2. Signal processing, which includes building library of signals at different flow conditions such as turbulent flow, laminar flow. Characterize typical dynamic signatures for varying conditions and establish classification criterion and thresholds.

3. Embedded, automated data collection and interrogation system integration.

4. Validation work on data collection, installation strategies, and decision making support tools.

5. Installation of field validation system at existing bridges.

In this research the effectiveness of signal processing and scour detection algorithms are explored for sensors of varying geometries to determine their sensitivity and the thresholds for false alarms and missed alert conditions at varying flow rates. To archive the above objectives and outcomes, several major tasks have been performed, which include extensive laboratory testing and data collection. The data gathered from laboratory experiments are utilized for this study. 


\section{Project Team and Task Allocation}

This thesis reports on research conducted as part of a larger research effort to develop and validate a robust and automated scour monitoring system and is a collaborative effort between Michigan Technological University and the University of Maryland. The thesis will focus on the portions of the project allocated to the author, however some broader context for the entire project will be presented here.

The research team at University of Maryland was responsible for scour sensor design, fluid interaction, and corrosion protection studies for the sensors. In the interest of facilitating some field testing in the early phase of the project, the research team at University of Maryland was also responsible for development of the smart scour-sensing posts with wired interface. The posts were constructed without the wireless and embedded data interrogation components as purely a data collection device. The data from the initial field testing was used to evaluate the behavior and survivability of the scour-post sensors in the field at Maryland.

The research team at Michigan Technological University performed signal processing tasks, design and development of embedded sensor interface, scour modeling and experimental design, and developing decision support visualization. The task involved laboratory scour detection studies to identify the limits of the proposed technology under challenging conditions, specifically low-flow conditions where signals appear similar to static conditions, development of signal processing algorithms, and establishing the thresholds that characterize different sensor states. The research team at MTU also developed an embedded data collection and interrogation hardware and software for smart scour-sensing posts, including wireless interface, and performed field testing of wireless scour-sensing system. 


\section{Literature Review}

\section{Introduction}

This chapter outlines the conventional instrumentation existing for monitoring and measurement of scour and their characteristics based on literature review of sensors used and instrumentation technologies that are utilized for monitoring scour at the specific site condition. During the past decade, monitoring concepts for structural systems have been subjected to a rapid development process. They play a huge role in the intervention planning of new and existing structures [6]. Similarly, in the past few decades a number of interesting methods for detection and monitoring of scour have been developed. This chapter briefly describes a few of the conventional methods that are widely used for scour monitoring and measurement.

Scour detection methods can be classified by several factors including function, purpose, measurement technologies, and instrumentation. They are classified into two categories according to instruments utilized is portable instrumentation methods and embedded instrumentation methods. Portable instrumentation method include diving, sounding rods, etc., and fixed or embedded instrumentation method consists of buried RF sensors, sonar, etc.

\section{Portable Instrumentation Method}

Portable instrumentation methods basically depend on visual inspections conducted by professionally trained inspectors with experience. Although this method of scour monitoring has served well for many years, this approach may not be the best to implement as the data from this method may not be very accurate and this approach can be dangerous in some cases.

Portable instrumentation methods are well suited for use by bridge maintenance and inspection crews for detecting scour conditions at piers and abutments and taking 
cross sections of the channel at problem bridges [9]. However, these methods have many drawbacks such as difficulty in mobilizing a crew of well trained workers and experienced supervisors and safety and requirement of traffic control for tests.

\section{Diving}

Diving is a simple method of scour monitoring in which a qualified bridge inspector performs a manual inspection of bridge underwater. Scour data can be collected from many locations using this method and the water clarity does not affect the data collection process. However, the drawback of this method is that it can be expensive and thus this method is mostly suitable only for worst case scenarios. It also includes considerable potential danger. Additionally, the data obtained by such visual inspections can contain a high degree of variability due to the subjectivity of the inspectors.

\section{Sounding Rods or weights}

Sounding-rod or falling-rod instruments are manual or mechanical gravity-based physical probes. In this method a qualified bridge inspector performs a manual inspection by dropping a rod or weight on the streambed to measure the sediment depth. The bottom of the rod needs to be wide in order to prevent the rod from penetrating the streambed caused by the weight of the rod and the vibration due to flowing water. Sounding rods tend to go through the streambed when the river bed consists of sand which influences their

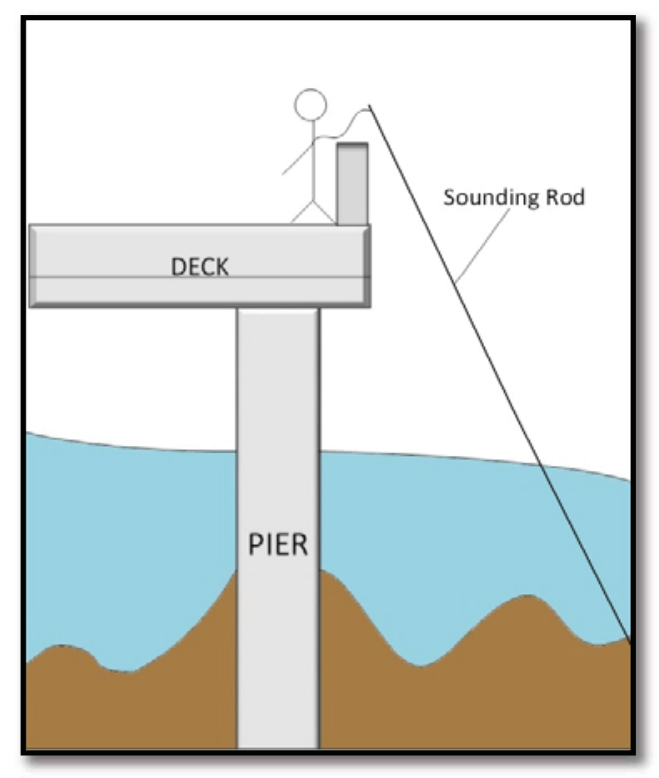

Figure 5. Sounding Rod 
accuracy. The main drawback of this method is the inaccuracy of the data samples acquired and the potential danger involved in this method. Moreover, this method can be expensive and does not have the ability of automated alerts [10].

\section{Embedded Instrumentation Method}

Fixed instrumentation methods, also known as embedded instrumentation of scour monitoring, include instrument that are placed in the vicinity of the bridge or in the bridge structure itself, typically at piers and abutments, to record data for the purpose of alerting the concerned personnel when the scour depth becomes excessive. The instrument is installed at preselected bridge sites to monitor and measure scour. Most commonly used embedded instrumentation monitoring methods include sonar based sensors, magnetic sliding collars, float-out devices, sounding rods and tilt meters. The type of fixed scour monitoring system adopted depends on the kind of information desired.

Embedded instrumentation generally measures scour only in the vicinity of the area where they are installed [11]. This method of scour monitoring does not require a field crew to be at site for monitoring or measurement or to record data. 


\section{Sonar-Based Sensors Method}

Sonar-based sensors are fixed instruments and are typically mounted at the pier and abutment locations. Sonar emits pulse waves and calculates the round trip travel time of a pulse from the riverbed to measure scour depth. Sonar sensors have the capability of measuring both scour and deposition of sediments. The major limitation of sonar as a scour measuring instrument is that their readings are negatively affected in water

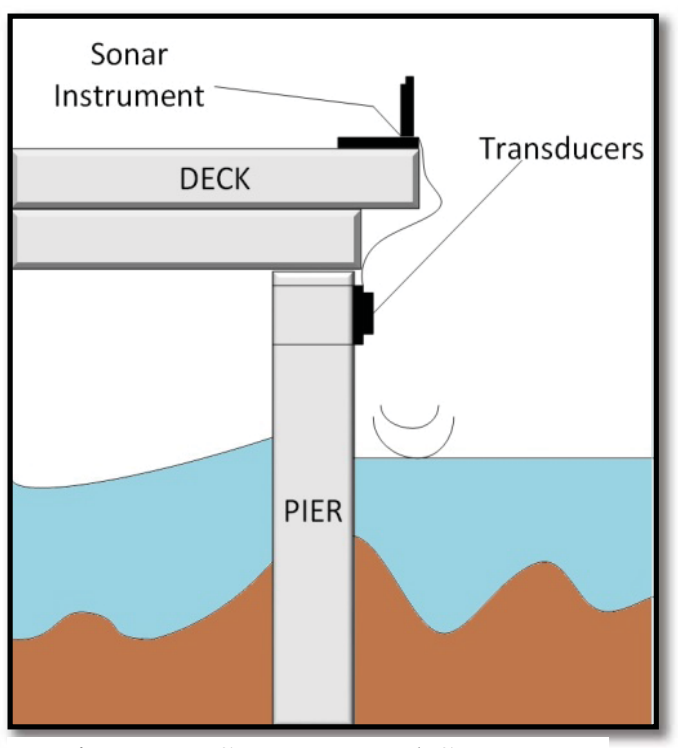

Figure 6. Sonar Based Sensors with high sediment and turbulent flow. High end sonars with high depth capacity and resolution can be expensive [12].

\section{Time Domain Reflectometer (TDR) Method}

Time domain reflectometer (TDR) based methods utilize similar method to sonar to measure scour. However, in TDR, a magnetic pulse is sent through sensor conduits and the magnetic pulse helps to determine the portion of the conduits buried in the stream bed. TDRs are capable of performing repeatable tests and are very robust. However, the tests conducted by this method require large amount of power,

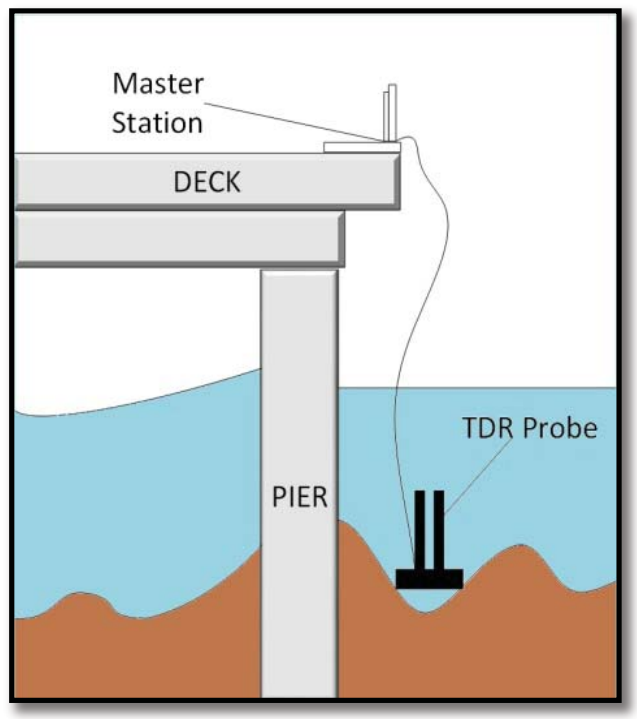
making them expensive. Furthermore, the Figure 7. Time Domain Reflectometer signal analysis instruments associated with Time domain Reflectometer are extremely complicated and are difficult to calibrate [13]. 


\section{Tilt -Meters Method}

Tiltmeters, also known as inclinometers, use a very simple method for scour measurement. Tilt-meters are fixed to a bridge structure such as an abutment or pier. When the structure leans or tilts due to scour, the Tilt-meters sense this change to measure scour. This method is relatively simple and is appropriate for extreme scour cases. However, this method measures extreme case scour and it can be difficult to distinguish between scour movements from the common movements of the structure caused by traffic, thermal, wind, and other causes [10].

\section{Magnetic sliding collar Method}

Magnetic sliding collars are another effective device used for detection of scour. This instrument consists of a heavy collar mounted on a magnetic rod. The rod is driven into the streambed and the collar rests on the streambed. During scour, as sediment is washed away from the streambed, the collar slides down the magnetic rod with the decreases in level. A

Figure 8. Tilt-Meter

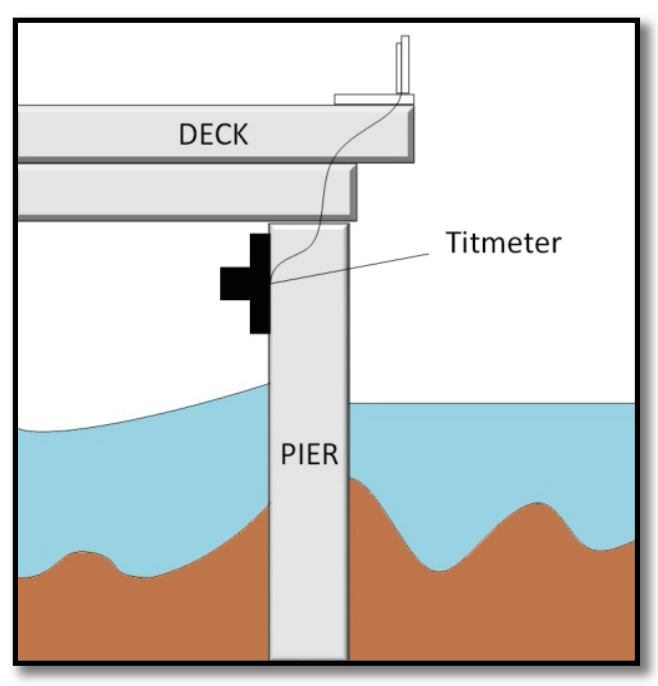
base station detects this change in height of the collar, which is used to deduce scour. It is a direct method of measurement and its reading are not affected by the quality of water. Although this method is very useful in measuring scour depth, the main drawback of this method is that its sensors can be used only once and they are not capable of measure sediment deposition [14]. 


\section{Buried RF Sensor Method}

Buried RF sensors are floating

electronic devices that are buried in sediment at predetermined depths. When the sediment is scoured away, the devices rise to the surface and transmit RF signal. The Base station senses signal and notes a scour event. The main advantage of this method is that it is not affected by the quality of water and it is a direct scour measurement. The main disadvantage associated with this method is that it uses single use sensors, making them

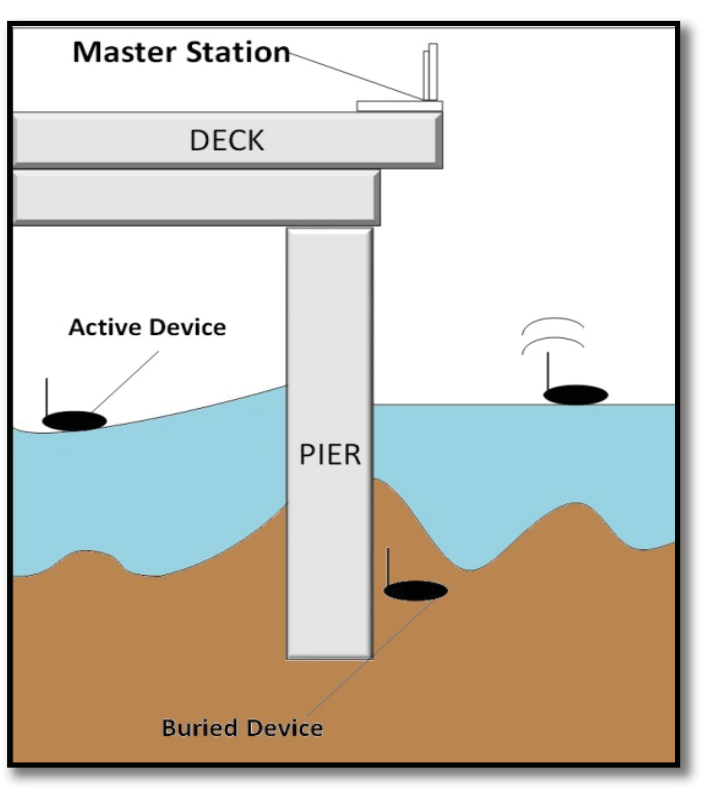

Figure 10. Buried RF Sensors costly. The sensors also require long lasting battery power to be able to transmit signals when they resurface to the top. Distinguishing between healthy devices and faulty ones can also be very difficult in this method [10].

\section{Vibration-Based Method}

This method utilizes the concept of measuring the fundamental frequency of a rod embedded in the riverbed. The inverse relationship between fundamental frequency and the length of the sensor rod is used for the detection of scour depth. It uses structural vibration sensors including accelerometers or fiber optic Bragg-grating (FBG) for use as the dynamic sensing element of the scour sensor. However, this method is yet to be fully validated. It is an ongoing research and tests are being performed on it [15]. 


\section{Acoustic Doppler Current Profiler Method}

This method is based on the Doppler Effect, i.e., the relative change in frequency of the wave. Acoustic Doppler Current Profilers use the Doppler Effect to measure the depth of water and to determine the velocity profile, which assists in scour forecasting. Scour is then determined from the velocity of the flow. It is an indirect method of sour measurement. Additionally, this method

of scour measurement is not feasible for areas with snow and the device used in

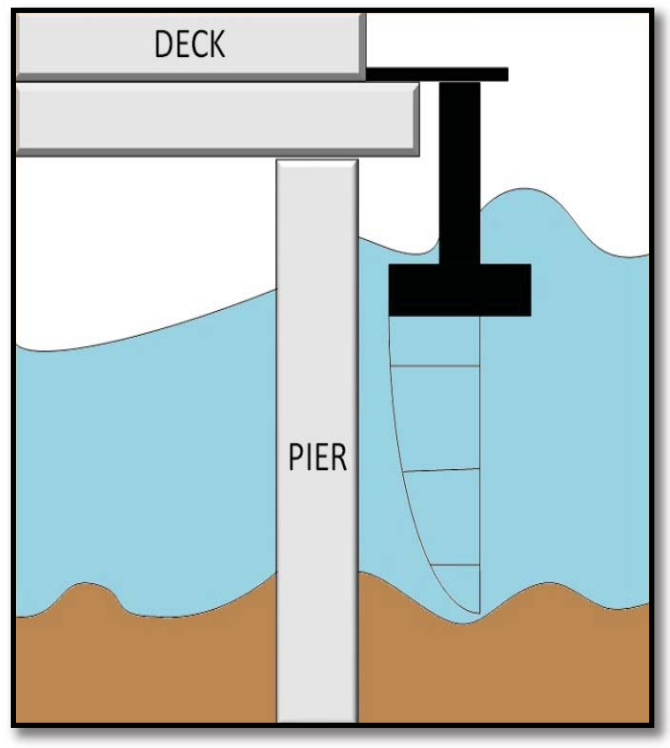

Figure 11. Acoustic Doppler this method are not rugged [16].

\section{Subsurface Geophysical Methods}

Geophysical instruments are based on wave propagation and reflection measurements. Geophysical instruments combine continuous seismic-reflection profiling and ground penetrating radar. They have the ability to provide accurate results and are capable of real time scour measurements. However, these instruments are expensive, very complicated to use, and require expertise to analyze the resulting data. The instrument is very hard to automate and it is not very effective in deep water. This method is mainly used after flood during lower flow conditions to locate scour holes and areas of infilling [17].

Other instruments for scour measurement include float out sensors and accelerometers. The accelerometer is used to measure the acceleration of the member it is attached to. It measures the vibration properties of the bridge which is caused by the change in support conditions of the bridge due to scour. Accelerometers are very easy to install and maintain but accelerometers require lot 
of power to transmit and require considerable post-processing. Float out sensors are similar to buried RF sensors.

An ideal scour monitoring and detection system should integrate all the advantages of various type monitoring systems to obtain more useful information on scour depth through direct monitoring and should be able to aid the decision-makers with the obtained information. In other words, they should be permanent, automated, robust, inexpensive, and should have a long life. Conventional scour monitoring instrumentation often necessitates costly installation and maintenance. They can also be vulnerable to debris damage during flooding. Often, the data from these instruments can be difficult to read and can be time consuming to process.

My research is focused on developing a low-maintenance system to detect and monitor scour development. The advantages of the system under study over traditional instrumentation are the ease of installation and the low maintenance.

The system studied in this research (automated scour detection array using magnetostrictive flow sensors) is automated, has the capability to capture transient events and can issue warnings. It is in continuous operation and the system uses low-power hardware components coupled with an appropriate power harvesting technology. It is highly robust (withstand adverse weather conditions) and inexpensive compared to other monitoring systems. Moreover, it uses low power and the data analysis is very simple. It harvests sustainable power by using solar cells. The sensors used are magnetostrictive sensors, which are attractive for scour measurement purpose due to their robustness and their low corrosion property. The proposed wireless monitoring system utilizes and takes advantage of a low-power wireless sensor network constructed from Narada wireless sensing modules and utilizes a cellular data link for secure data transfer. 


\section{Hardware and Experimental Setup}

\section{Introduction}

In this study, a low-power wireless sensor network constructed from Narada wireless sensing units is used to create a wireless monitoring system with enhanced longevity in the field. The power source for the system is provided via solar energy. The system under study deploys and tests an embedded array of sensors located at varying heights near bridge foundations, and determines the sediment depth and profile around the waterway in real time. The hardware of the system consists of bio-inspired magnetostrictive flow sensors that detect water flow by bending. The Narada wireless sensing unit converts analog voltage output signals from sensor into digital signal, collects and analyzes data, and relays the analyzed data to remote base station. A base station system which includes a Narada Base Station, a single board computer, a cellular data link, and a power source, aggregates the data collected from the multiple units, creates a decision file, and sends scour alerts to relevant authorities using a cellular link.

\section{Sensors}

The magnetostrictive flow sensors utilized in this study were developed at University of Maryland. Different ruggedness tests and various methods of sensor protection have been carried out at University of Maryland. During the course of this study three different sensors were (Image Source www.flickr.com, See Appendixused: 1) whisker-inspired

Figure 12. Marine Animals with Whiskers

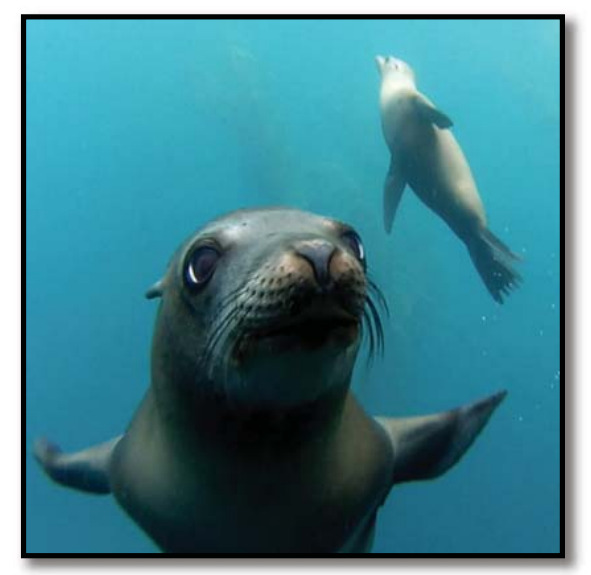

A for Permissions

sensors, 2) airfoil sensors, and 3) seaweed sensors. 


\section{Whisker-inspired magnetostrictive Galfenol sensors}

The whisker shaped sensors are inspired by the whiskers of marine animals. Whiskers provide marine animals with important sensory information about the world around them. The configuration of the whisker sensor is very simple and easy to assemble. It consists of five main components: 1) Galfenol whisker, 2) GMR sensor, 3) clamping fixture, 4) small permanent magnet, and 5) low power operational amplifier. Figure 13 shows the typical flow sensing components.

The whisker-inspired flow sensors are constructed using a magnetostrictive Galfenol wire. Magnetostrictive materials have the ability to transduce or convert magnetic energy to mechanical energy and vice versa. Galfenol is an alloy of iron and gallium which has corrosion properties that are similar to those of steel, and four times less than iron [18].

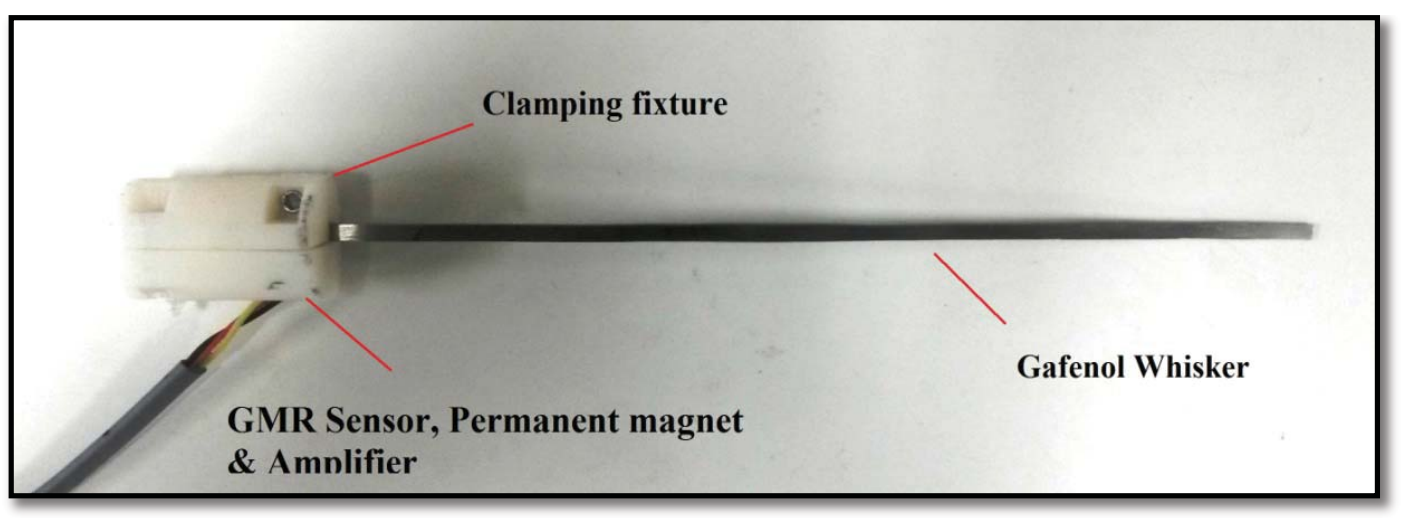

Figure 13. Bio-inspired Magnetostrictive Whisker Sensor

One end of a Galfenol whisker is fixed and the other end is free to deflect under fluid flow-induced drag forces (Figure 14). The bending causes stress near the fixed end of the whisker which produces local change in the orientation of the magnetic field, which is followed by a global change in the magnetic flux density in the whisker. A giant magnetoresistance (GMR) sensor at the fixed end of the whisker senses this change of magnetic field and converts it into electrical signal, which is amplified and transmitted. The GMR sensor is soldered to a printed circuit board 
and includes a Burr Brown INA118 instrumentation amplifier. A permanent magnet is in contact with the whisker to align magnetic field along the whisker when it is not deformed. Different strength magnets can be used as long as a proper magnetic field strength is achieved at the root of the whisker, ensuring internal magnetic dipole rotation in response to movement of the whisker without saturating the GMR sensor [18]. The Galfenol wire that is used in the transducer is inexpensive to produce and the magnetic field reader can be harvested from computer hard-disk drive. The other components used in the transducer are easily available and inexpensive.

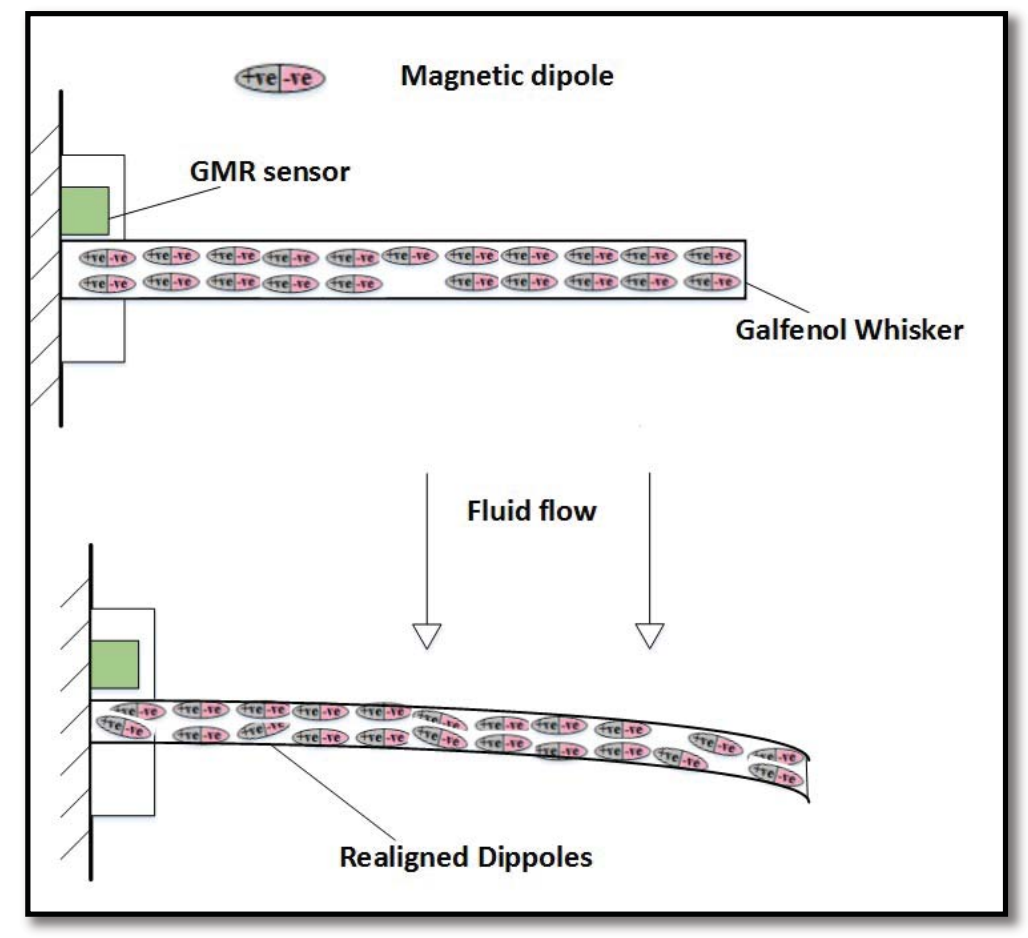

Figure 14. Working Principle of Magnetostrictive Sensor (Image by Author) 


\section{Airfoil magnetostrictive Galfenol Sensors}

Airfoil sensors are an improved version of whisker-inspired magnetostrictive Galfenol sensors. Airfoil sensors work on the exact same concept as the bio-inspired whisker flow sensors. However, the airfoil sensors are much more robust compared to the whisker inspired sensors. A wax layer in a shape of an airfoil was added to the sensors

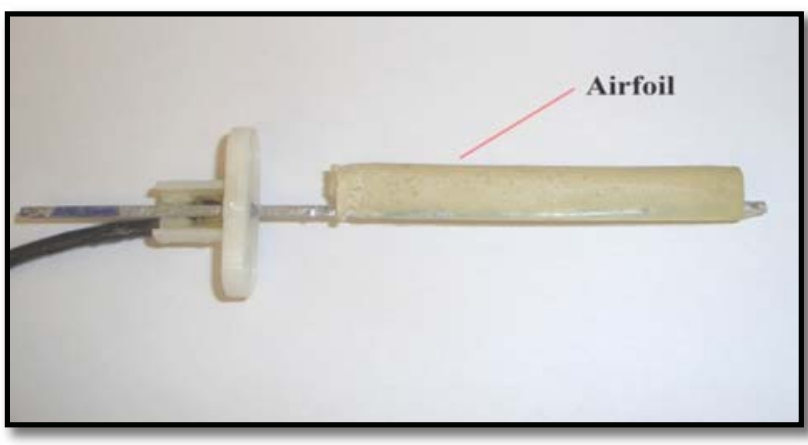

Figure 15. Airfoil-type Sensor (Image by Author)

to improve its sensitivity (Figure 15).

\section{Magnetic Seaweed Sensor}

Seaweed type sensors are the latest sensor developed after the airfoil type sensors. The seaweed sensors have undergone quite a few changes compared to predecessors. The airfoil and Galfenol whiskers have been replaced by a strip of thin fabric material. The strip has a small magnet attached to it. The strip deflects under fluid flow, causing change in the magnetic field. The

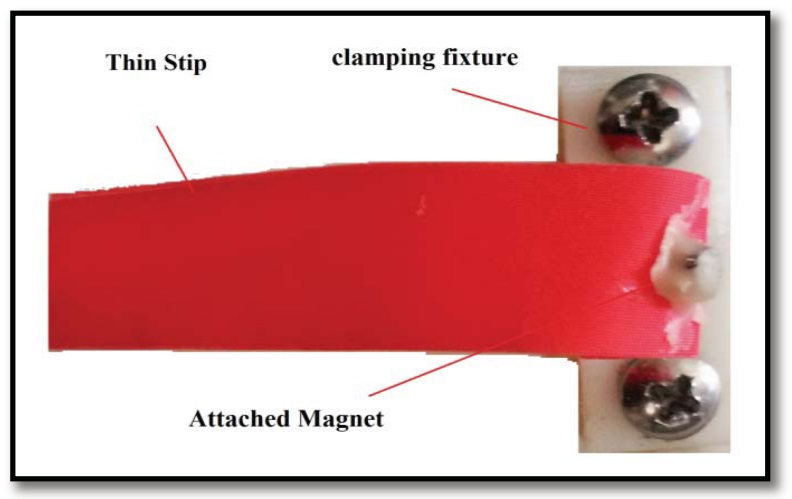

GMR sensor at the fixed end of the Figure 16. Seaweed-type Sensor (Image strip, inside the clamping fixture, by Author) detects this change of magnetic field and converts it into electrical signal. A seaweed-type sensor is depicted in Figure 16. 


\section{Wireless sensing unit for scour sensing posts}

For a wireless network design to excel, specific hardware requirements must be satisfied to tackle the inbuilt challenges of wireless network operations. It must have low power consumption, low latency, and the ability to quickly process data and execute control algorithms. For wireless sensor

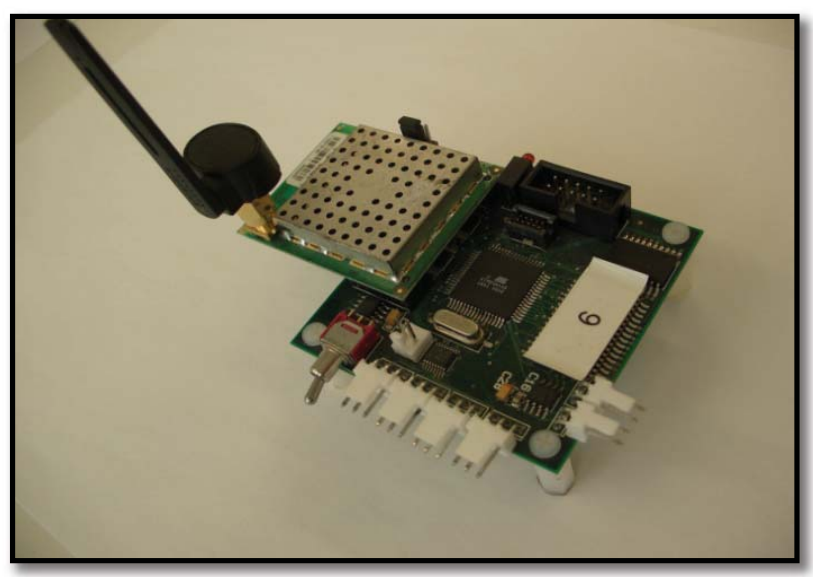

Figure 17. Narada WSU (Image by Author) nodes to communicate with one another a digital wireless radio is required and as

the output from most sensors is analog, the signal at some point must be converted into digital signal domain in order to be transmitted. Furthermore, the tasks performed by the sensing node such as sampling computations and communications requires time-specific coordination [19]. To fulfill these requirements the wireless sensing unit must contain the following modules: 1) sensing interface; 2) communication interface; 3) computational core; and 4) actuation interface. The wireless sensing interface used in this research is the Narada wireless sensing unit (see Figure 17) and are produced by Civionics Inc are easily available for a Figure 18. Antenna and Radio reasonable price, which make the proposed Daughter Board (Image by system inexpensive.

Author) 
The Narada WSU (wireless sensing unit) is a low-power wireless sensor node designed explicitly for the monitoring of civil infrastructure systems and has been successfully used in many types of structures in the past including buildings, bridges, wind turbine towers, and naval vessels. It has been designed for applications requiring high resolution data collection, and/or real-time control [20].

The Narada features ADS8341 16-bit digitalization of analog sensor data with four analog input channels that can read analog signals ranging from 0 to 5 Volts. The core of the wireless sensor is an 8-bit ATmega128 microcontroller that is responsible for the overall node operation including data storage. The ATmega128 is an 8 bit, low power microcontroller that has $128 \mathrm{kB}$ of flash memory to store data and embedded algorithms, and $4 \mathrm{kB}$ of electrically erasable programmable read only memory (EEPROM). The maximum number of sampling points that can be saved in the core is limited by the node's $128 \mathrm{kB}$ SRAM bank. The Narada features an IEEE 802.15.4 compliant wireless modem, the Chipcon CC2420, which adopts the 2.4 GHz IEEE 802.15.4 radio standard (Zigbee). The transceiver is designed with a power amplifier (Figure 18) to boost the communication power of the node for the Zigbee long-range communication (i.e., $700 \mathrm{~m}$ line-of-sight). The wireless sensing unit used utilizes low-power wireless signal network to communicate with local base stations. However, every sensor board has to be able to reach the base station in order to transfer data, limiting the distance between the base station and the sensor that is farthest away.

Any type of sensor can be attached to the WSU and external sensors on the Narada system allow easy removal in case the sensors are damaged or faulty. However, the drawback of Narada is that it has inflexible power management schemes and relatively slow floating-point calculations emulated in its fixed point microcontroller.

The Narada wireless sensing unit contains an inbuilt actuation interface. The actuation interface allows the network to engage in active sensing and to be applied 
to a wireless feedback control application. The actuation interface consists of a Texas Instruments DAC7612 2 channel 12-bit DAC capable of outputting analog signals from 0 to $4.1 \mathrm{~V}$, with a resolution of $1 \mathrm{mV}$.

Table 1. Technical Specification of Narada WSU [21]

\begin{tabular}{|c|c|c|c|}
\hline Dimensions & $69 \mathrm{~mm} \times 72 \mathrm{~mm} \times 12$ & \multicolumn{2}{|c|}{ CPU } \\
\hline Base station to $\mathrm{PC}$ & RS-232,USB & Processor & Atmel \\
\hline \multicolumn{2}{|c|}{ Voltage Actuation } & FLASH & $128 \mathrm{kB}$ \\
\hline Output Channels & 2 & EEPROM & $4 \mathrm{~KB}$ \\
\hline DIGITAL to Analog & 12-bit resolution & SRAM & $4 \mathrm{~KB}$ \\
\hline Maximum output & $15 \mathrm{~mA}$ & $\begin{array}{l}\text { External } \\
\text { SRAM }\end{array}$ & $128 \mathrm{kB}$ \\
\hline \multicolumn{2}{|c|}{ RADIO } & \multicolumn{2}{|c|}{ Power Consumption } \\
\hline Transceiver & TI CC2420 & Current & $10 \mathrm{~mA}$ \\
\hline Frequency Band & $2.4000-2.4835 \mathrm{GHz}$ & Current & $30 \mathrm{~mA}$ \\
\hline Data Rate & $250 \mathrm{~kb} / \mathrm{s}$ & Current & $52 \mathrm{~mA}$ \\
\hline Range (line of sight) & $500-600 \mathrm{~m}$ & Input & $6.0-9.0 \mathrm{~V}$ \\
\hline \multicolumn{4}{|c|}{ Data Acquisition } \\
\hline Input Channels & 4 single ended/2 & DC Sensor & $+5 \mathrm{~V} \mathrm{DC}$ \\
\hline Analog to Digital & 16 bit resolution & Real time & 1500 \\
\hline Data Storage Capacity & $128 \mathrm{kB}$ & Maximum & 10000 \\
\hline
\end{tabular}




\section{Assembled smart scour sensing posts}

The smart scour-sensing posts are made of $3.96 \mathrm{~m}$ (13ft) long PVC pipe (steel is also an option). The bottom 1 foot of the posts was filled with concrete so that the posts could be easily driven into the ground near abutments or placed using an auger. Four transducer elements with $0.91 \mathrm{~m}$ (3ft) spacing to each other were mounted on the posts. Polycase waterproof enclosures made of polycarbonate and (YH-080604 YH Series Hinged Waterproof NEMA Electrical Enclosures) of dimensions 8.59 x $6.59 \times 5.11 \mathrm{in}$. house the embedded data collection and interrogation electronics, power manager and a long-lived battery pack packaged were mounted on top of the post using a mounting flange assembly. The polycase used are easily available and inexpensive. The tops of these posts are designed to be located in air to facilitate wireless operation. Low-power components and use of sleep mode were employed to extend the battery life. Figure 19 depicts the electronics in the protective enclosure and Figure 20 shows a packaged smart scour-sensing post prepared for field installation. 


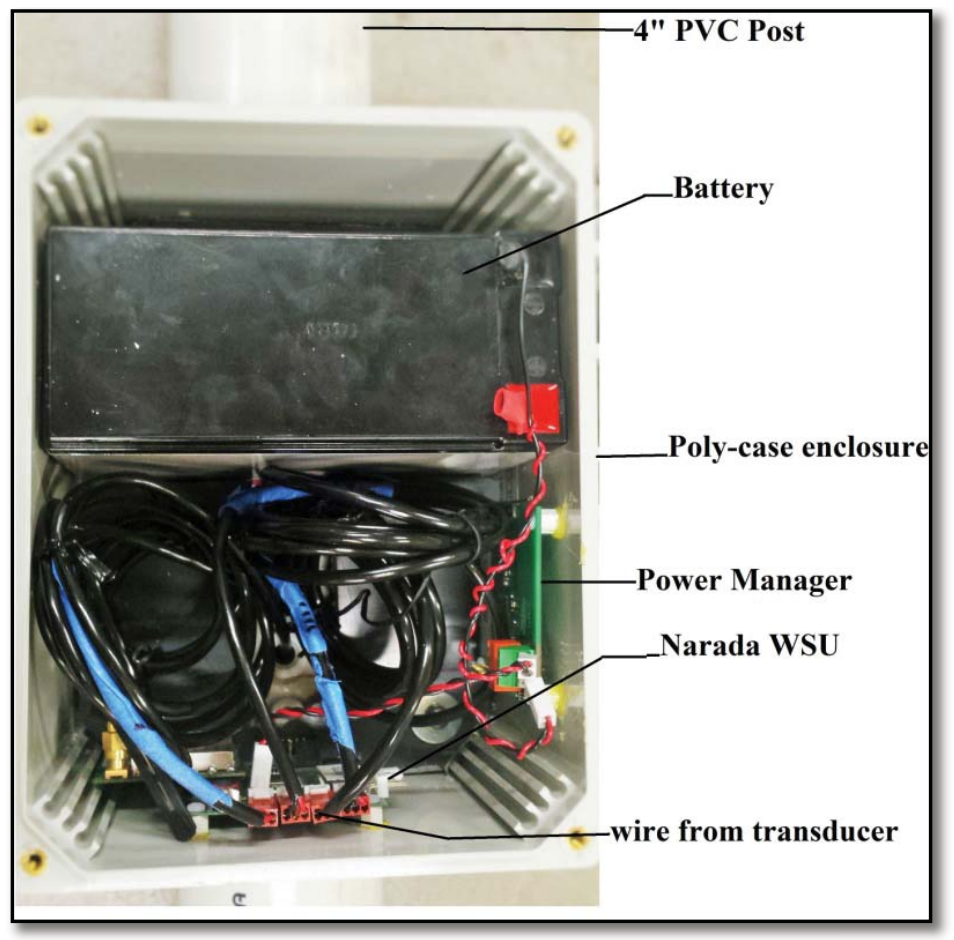

Figure 19. Post Electronics Packaged in Polycase Enclosure (Image by Author)

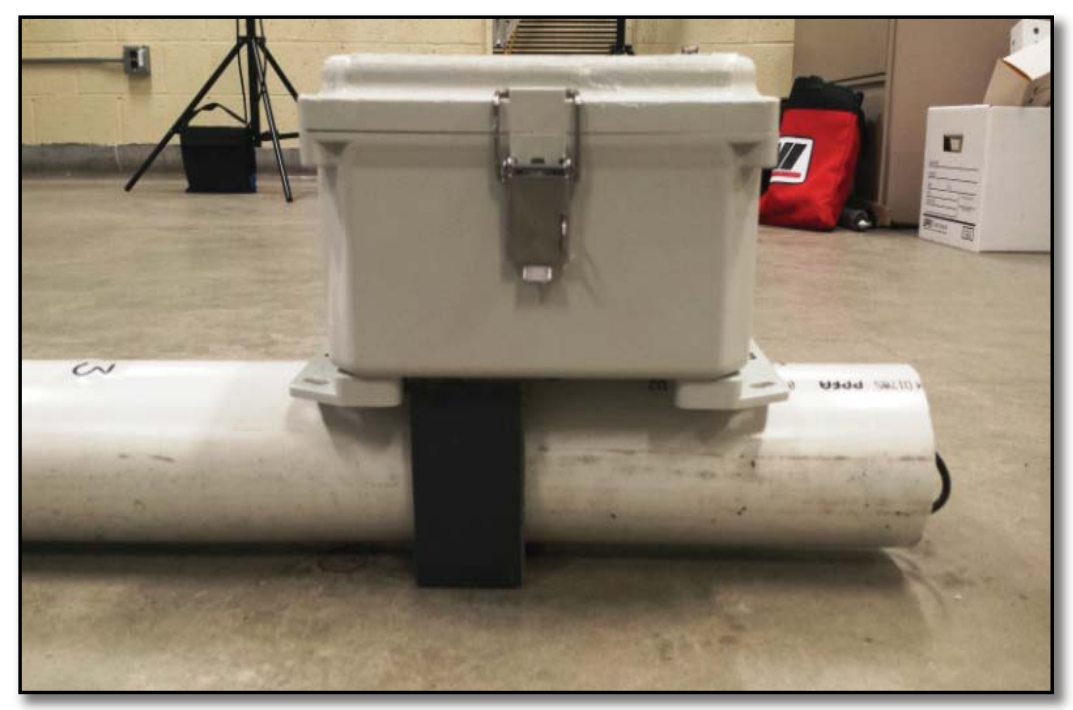

Figure 20. Packaged Smart Scour-sensing Post (Image by Author) 


\section{Base Station}

The base station aggregates the data from multiple on-site posts and it creates a decision file in accordance to the data received from the wireless sensing units. The base station then relays the file to relevant authorities using a cellular data link to aid them to take suitable required action.

The base station is composed a Narada Base Station, Single Board computer (SBC), cellular data link, and a power supply for the setup.

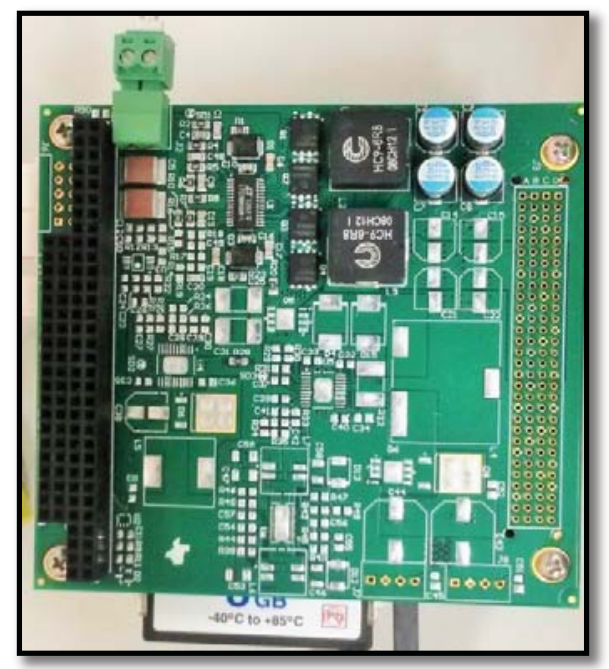

Figure 21. Single Board Computer for Base Station (PPM-LX800-G)

(Image by Author)

\section{Single Board Computer}

The Single board computer used for this research is an industrial platform that can safely operate in harsh conditions. The PPM-LX800-G manufactured by WinSystems (Figure 21). The PPM-LX800-G is a highly integrated, PC/104-Plus single board computer (SBC) designed for embedded, space limited, low power applications. The PPM-LX800-G is well suited for rugged applications requiring excellent processor performance in an embedded PC design. Its low power dissipation permits fan-less operation from $-40^{\circ}$ to $+85^{\circ} \mathrm{C}$ [WinSystems] [22].

The server consists of a low-power single board computer (SBC) that operates Linux (Ubuntu 11.10) 
Table 2. Technical Specifications of SBC (WinSystems) [22]

\begin{tabular}{|c|l|}
\hline Processor & LX800 @ 0.9W with 500MHz system clock \\
\hline Memory & $\begin{array}{l}\text { 256KB of L2 cache Up to 1GB with a 200-pin SDRAM } \\
\text { in a SODIMM connector }\end{array}$ \\
\hline $\begin{array}{l}\text { Network } \\
\text { Interface }\end{array}$ & One 10/100 Mbps using the Intel 82551ER LAN \\
\hline Storage & One Compact Flash socket supports Type I or II devices \\
\hline Power & $+5 \mathrm{~V}$ required, 1.2A typical \\
\hline
\end{tabular}

\section{Cellular Data Link}

A $3 \mathrm{G}$ cellular modem is included in the server for the communication of data. The cellular data link used is the Pantech 4G LTE USB Modem UML290 (Figure 22). The UML290 is designed with manufacturer designed power management and system overhead reduction functions to take advantage of the USB interface to reduce power consumption. It contains an internal antenna which is designed to optimize data transfer rate and sensitivity to network signals. The UML290 has typical download speeds of 5 to $12 \mathrm{Mbps}$ in mobile broadband coverage area. The UML290 comes equipped with a fold-away, 180

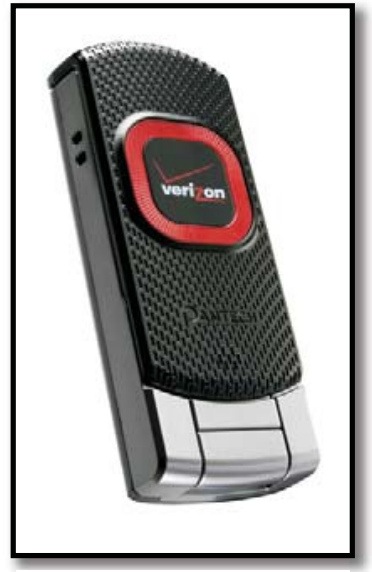

Figure 22. UML 290 (Image by Author) degree rotating USB connector. The UML290 is also well-suited for use with Linux, with minimal driver installation effort required by the user [23]. 
Table 3. Technical Specifications UML 290 [23]

\begin{tabular}{|c|l|}
\hline Broadband & $\begin{array}{l}\text { 4G LTE Mobile Broadband and Mobile Broadband (Rev. } \\
\text { A) capable }\end{array}$ \\
\hline Memory & Qualcomm MDM9600 \\
\hline Network bands & $\begin{array}{l}\text { CDMA 1xEV-DO Rev. A/Rev. 0: 800/1900 MHz, LTE } \\
\text { CDMA (700MHz) }\end{array}$ \\
\hline Antenna & External Antenna Connector \\
\hline
\end{tabular}

\section{Narada Base Station}

The Narada base station (Figure 23) is an IEEE802.15.4 data transmission device that connects to a PC via USB and serves as the base station's low-power link to the on-site posts. The Narada Base Station is produced by Civionics Inc. The Narada Base station is a wireless data acquisition hub built around the Atmel ATmega128 microcontroller. The ATmega128 is an 8 bit, low power microcontroller. The Narada

Base station connects to the PC through an Figure 23. Narada Base available USB port and can facilitate data Station (Image by Author) collection, real-time control, and network maintenance tasks (Civionics) [21]. 
Table 4. Narada Base Station Technical Specifications: (Civionics) [21]

\begin{tabular}{|c|c|}
\hline Dimensions & $3.0 " \times 2.0 " \times 1.5 "$ \\
\hline $\begin{array}{c}\text { Base station to PC } \\
\text { interface }\end{array}$ & RS-232,USB \\
\hline CPU & \\
\hline \multirow{6}{*}{ Processor } & Atmel ATmega128 \\
\hline & FLASH 128 kB \\
\hline & EEPROM $4 \mathrm{kB}$ \\
\hline & SRAM $4 \mathrm{kB}$ \\
\hline & External SRAM $128 \mathrm{kB}$ \\
\hline & External Clock Speed $8 \mathrm{MHz}$ \\
\hline RADIO & Transceiver TI CC2420 \\
\hline Frequency Band & $2.4000-2.4835 \mathrm{GHz}$ \\
\hline Data Rate & $250 \mathrm{~kb} / \mathrm{s}$ \\
\hline Range (line of sight) & $600 \mathrm{~m}$ \\
\hline
\end{tabular}

\section{Assembled Base Station}

Polycase waterproof enclosures (YH-161407 YH Series Hinged Waterproof NEMA Electrical Enclosures) of dimensions 16.59 x 14.59 × 8.24 in. were used for the base station, housing the Narada base station, single board computer, a wireless communication interface, power manager, and battery pack. The base-station units were fit with solar panels for battery recharging. Figure 24 shows the base station assembly and Figure 25 shows the solar panel that was used to recharge the base station battery. 


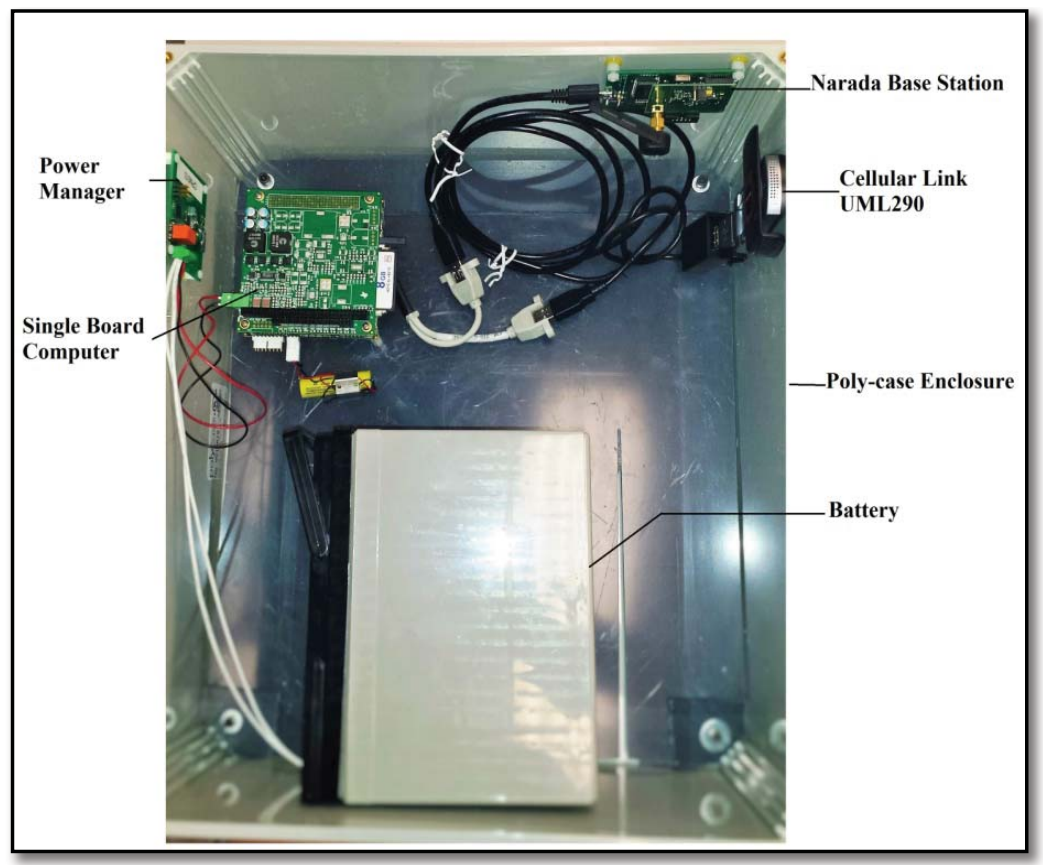

Figure 24. Base Station Electronics in Protective Enclosure (Image by Author)

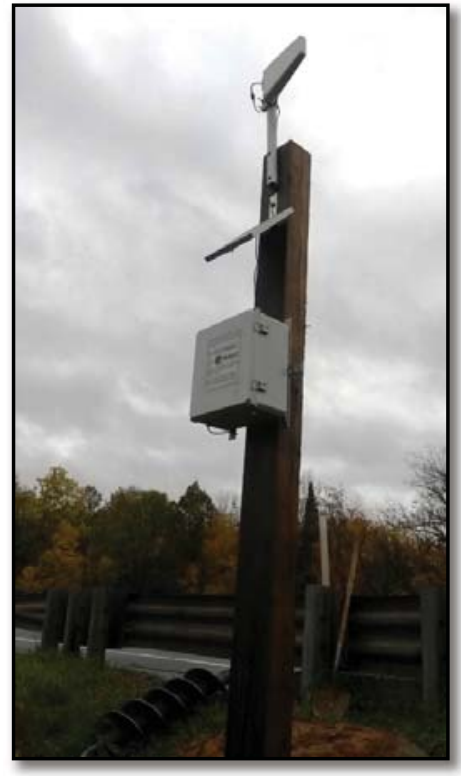

Figure 25. Assembled Based Station Installed in the Field (Image by Author) 


\section{Detection Algorithm}

This chapter covers the implementation details of the algorithm, showing how it has been executed. The algorithm was developed by R. Andrew Swartz and Andrew T. Zimmerman.

\section{Introduction}

This thesis presents the implementation details of the river bed detection algorithm, showing how it has been executed. The riverbed detection algorithm analyzes the raw sensor data from each buried whisker transducer and classifies the sensor output as static state, dynamic state, or faulty. Those sensors that are static are assumed to be buried in sediment while those that are dynamic are considered to be above the riverbed or located in a scour hole. In this effort, simple algorithms that give good sensitivity even in low-flow conditions are preferred in order to allow for more reliable autonomous operation as well as reduce the computational effort required to classify the data (which will save battery energy within the post). The algorithm must also have minimal memory requirements to allow it to be executed and embedded in the resource-constrained, low-power wireless sensor units (WSUs) that form the computational core of the smart scour-sensing posts developed for this study. Direct data interrogation within the post will eliminate the need to communicate copious amounts of raw data wirelessly at bridge sites, improving network and system resilience and reducing energy consumption. In addition to the low-power WSUs, the algorithm will be implemented on single board computers at some sites where wireless operation is not an objective. In these implementations, energy and computational resources are less limited. 


\section{Objectives for autonomous detection algorithm}

The design of the algorithm had several objectives:

- Simplicity

- Minimal memory utilization

- Fast execution time

- High sensitivity

- Repeatability

- Error detection

\section{Platforms}

The project will use both low-power WSUs as well as more capable SBCs as data acquisition and processing platforms. Narada wireless sensor node comes with a custom written embedded operating system. The operating system simplifies the process of the wireless sensor for the user and serves as an intermediary software layer between hardware and software written for data interrogation. Within the custom operating system, additional engineering algorithms for data interrogation may be used with a number of algorithms already developed in an existing library. Some of the algorithms that were utilized for this project included computation of basic statistical measures, the fast Fourier transform (FFT), and least-squares approximations of time-series models for input/output data (e.g., AR, ARX, ARMA, etc.).

To support additional platforms (and for debugging purposes), these algorithms were ported to Linux as well as Windows. Since, for implementation in the SBCs selected for this study, the Linux operating system (Ubuntu 11.10) is being utilized, the final integrated detection algorithm was ported to Linux. 


\section{Operation}

In this study, a central based station (composed primarily of a SBC) was used to coordinate the activities of the wireless monitoring system and to serve as storage of network measurement data of the system. To initialize the Narada WSUs and execute the riverbank detection algorithm, three server setup files are used. The first file, default_settings.dat, is a text file containing DAQ parameters. It is created by the user, processed by an executable server program running on the $\mathrm{PC}$, and wirelessly transmitted to the network over the Narada IEEE802.15.4 receiver board connected to the SBC USB port. The default_settings.dat file allows the user to modify the IEEE802.15.4 network settings defined for the wireless sensor network including the communication channel, personal area network (PAN) ID, and the server node ID. A second file, called DAQ_settings.dat, is used to set data acquisition parameters, including the sampling frequency, total sampling time for a test, the number and ID of the WSUs participating in a test, and the WSU data channels to be used [20]. A final setup file, analysis_settings.dat, is defined to establish the thresholds to apply to each sensor channel that help the algorithm to differentiate between static and dynamic signals (these thresholds will vary according to the transducer used).

The base station initiates data acquisition from all WSUs within the local network at the beginning of a scour detection cycle. WSUs within scour posts collect and store time history data from all transducers on the posts. At the end of the test, the base station ensures that the test has completed and then requests that the WSUs perform data processing on their locally collected data, including computing basic statistical measures and frequency-domain analysis using an FFT and rudimentary digital filtering. The results of these algorithms are reported to the base station which then classifies each signal according to the rules defined in the setup file. The results from each channel are then uploaded via cellular data modem to a central server to be presented to the user. An overview of this process is shown in Figure 26. 
Additional details of the individual steps are provided in subsequent sections of this report [24].

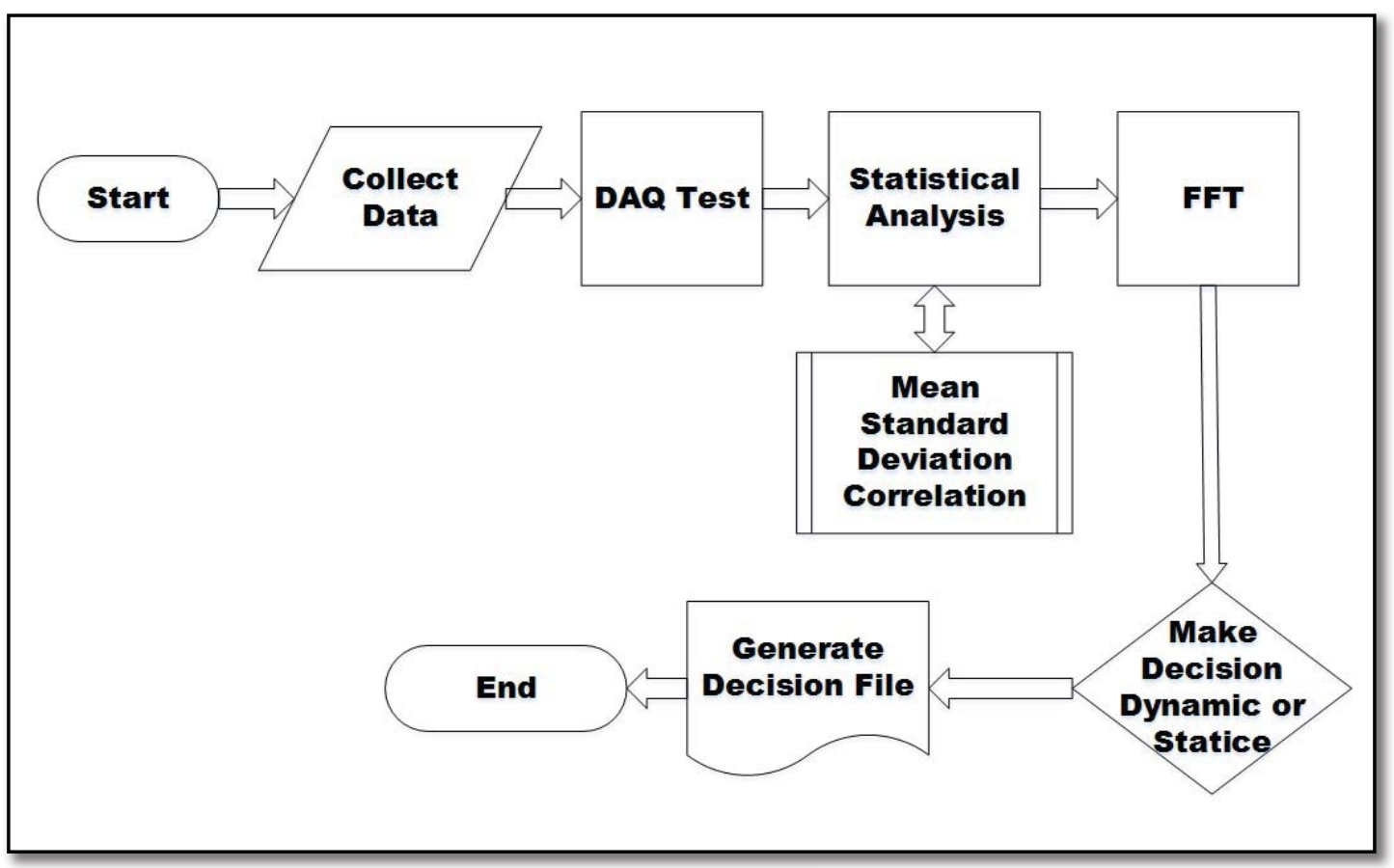

Figure 26. Basic Algorithm Steps 


\section{Data acquisition (DAQ)}

Data acquisition module, written for the embedded operating system provides the wireless sensing unit with the ability to collect and store data and to stream data in real time to a server; the former being the most useful for this application. The DAQ collects data from the node analog-to-digital converter (ADC) and stores it in SRAM. Data processing activities take place within the sensor node and only processed results are reported to the base station. Bridge managers may make a request from the remote visualization client to view raw sensor data from the sensors in upcoming detection cycles. In this case, the base station will request raw data from the WSUs as well, collecting it and uploading it to the remote server via cellular data link [20].

\section{Statistical computation}

Statistical modules have been added to allow the WSU to perform statistical analysis of the data collected. The algorithm is written so that the WSU can calculate the mean, standard deviation, correlation, and the cross correlation between the sensors. The data are assumed to be Gaussian distributed to calculate the statistical measures. These measures (particularly standard deviation) can be compared to a threshold to determine whether the transducer is measuring static or dynamic signals. In addition, cross correlation measurements can be helpful in identifying cross-talk and other indications of faulty sensor channels. The following are equations used to calculate the statistics. 
Calculate the mean for all channels (for $n$ samples):

$$
\mu x=\left(\frac{1}{n}\right) \sum_{\mathrm{i}=1}^{\mathrm{n}} \mathrm{Xi} .
$$

Calculate the Gaussian standard deviation for all channels:

$$
\begin{gathered}
\operatorname{Var}=\left(\frac{1}{n-1}\right) \sum_{\mathrm{i}=1}^{\mathrm{n}}(\mathrm{Xi})^{2} ; \\
\sigma=\sqrt{\text { Variance }} .
\end{gathered}
$$

Calculate covariance between all channels:

$$
\operatorname{cov}(X, Y)=\frac{\sum_{i=1}^{n}\left(X_{i}-\mu_{X}\right)\left(Y_{i}-\mu_{Y}\right)}{n-1} .
$$

Calculate correlation coefficients:

$$
r_{X, Y}=\frac{\operatorname{cov}(X, Y)}{\sigma_{X} \sigma_{Y}} .
$$

\section{Fast Fourier transform and frequency-domain analysis}

The fast Fourier transform (FFT) modules have been written for the embedded operating system to provide the wireless sensing unit with the capability to perform frequency-domain analysis of the data. The FFT previously included has been altered to make it able to compute FFT for one to four channels. It also calculates the magnitude and phase angle of the FFT, which is used to define the static/dynamic signal threshold. To conserve memory within the WSU, only the lower half of the FFT is stored in memory (i.e., those values below the Nyquist frequency) because there is no independent information contained in the remaining portion of the FFT results. For dynamic signal detection, only low-frequency values of the FFT are owing to the limited bandwidth of the fluid-coupled transducer output. Laboratory studies revealed that frequencies above $10 \mathrm{~Hz}$ generally did not 
contain signals corresponding to the vibrations of the whisker sensors in their submerged state. Rudimentary digital filtering of the signal above $10 \mathrm{~Hz}$ was performed and the sum of the remaining FFT magnitude is used as the primary indicator of the presence of dynamic signals, with large sums indicating dynamic behavior. High levels of signals in frequencies above $10 \mathrm{~Hz}$ and poor signal-tonoise ratios measured in the frequency domain were used as indicators of sensor faults.

\section{Establishing Thresholds}

The scour monitoring system under study functions by differentiating between static and dynamic flow signals. When plotting the time domain response and frequency domain response of flow sensors at high flow conditions and low flow conditions it was observed that it was easy to differentiate between static and dynamic flow signals in high velocity flow. A large increase in the standard deviation and magnitude of the frequency-domain response was observed in dynamic signals when compared to the static signals (Figure 27). However, under low-flow conditions, the standard deviation was largely insensitive and it was observed that magnitude of the frequency-domain response more sensitive and better for defining threshold for low flow conditions (Figure 28). 

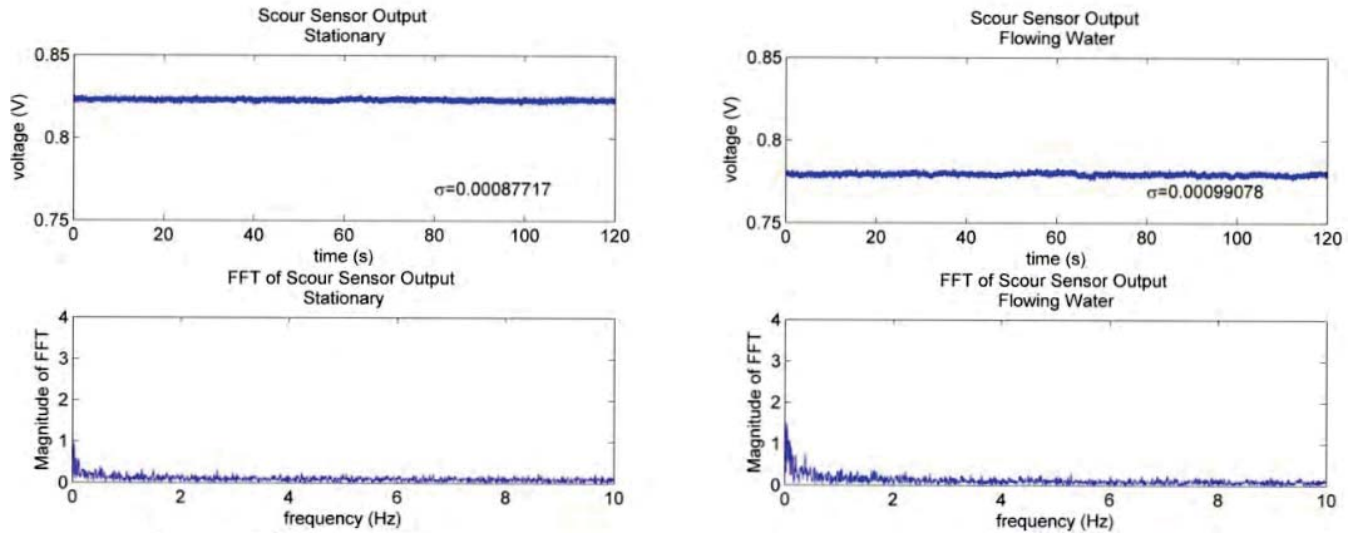

Figure 27. Low Flow Condition Time Domain and Frequency Domain Response for Buried and Unburied sensors
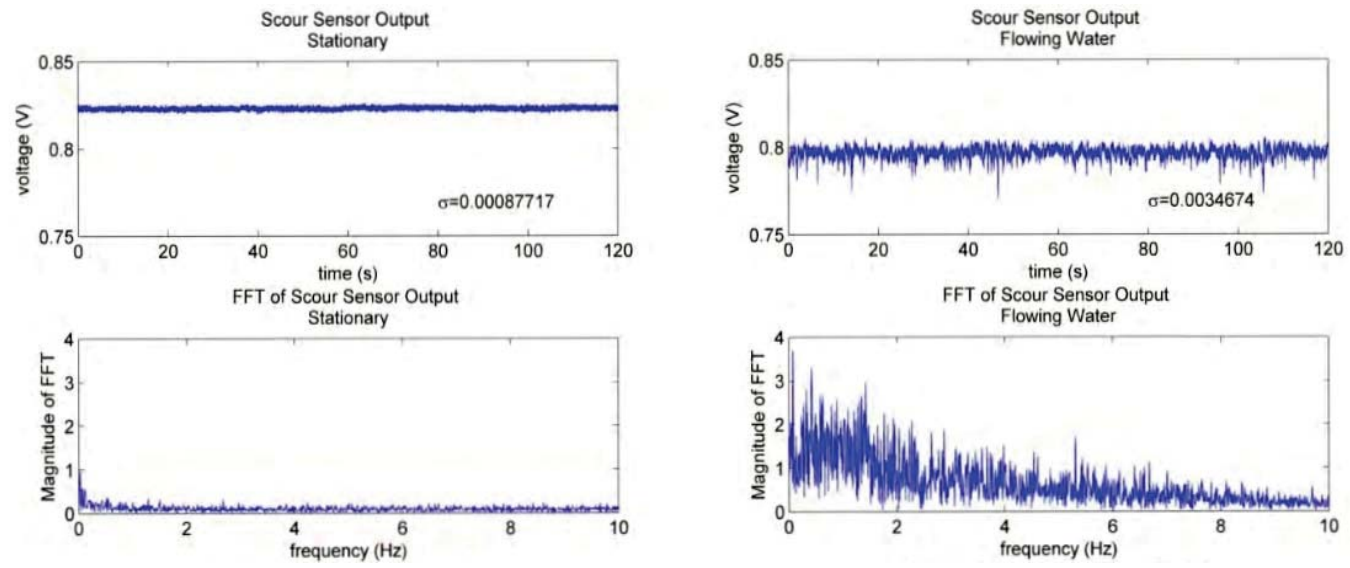

Figure 28, High Flow Condition Time Domain and Frequency Domain Response for Buried and Unburied Sensors 

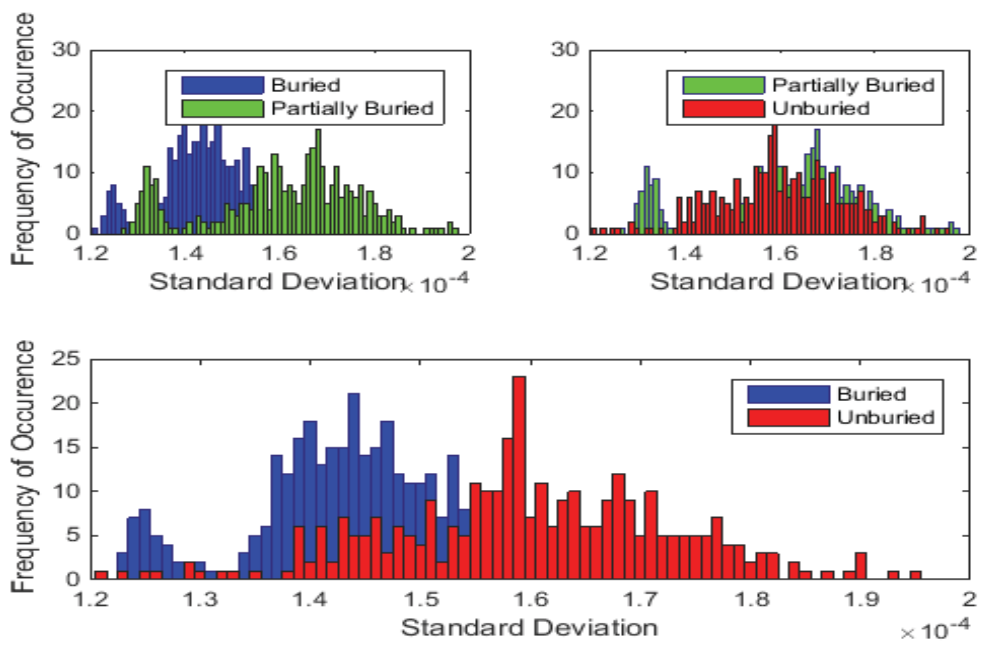

Figure 29. Histogram of Standard Deviation for Airfoil Type Sensors at Different States
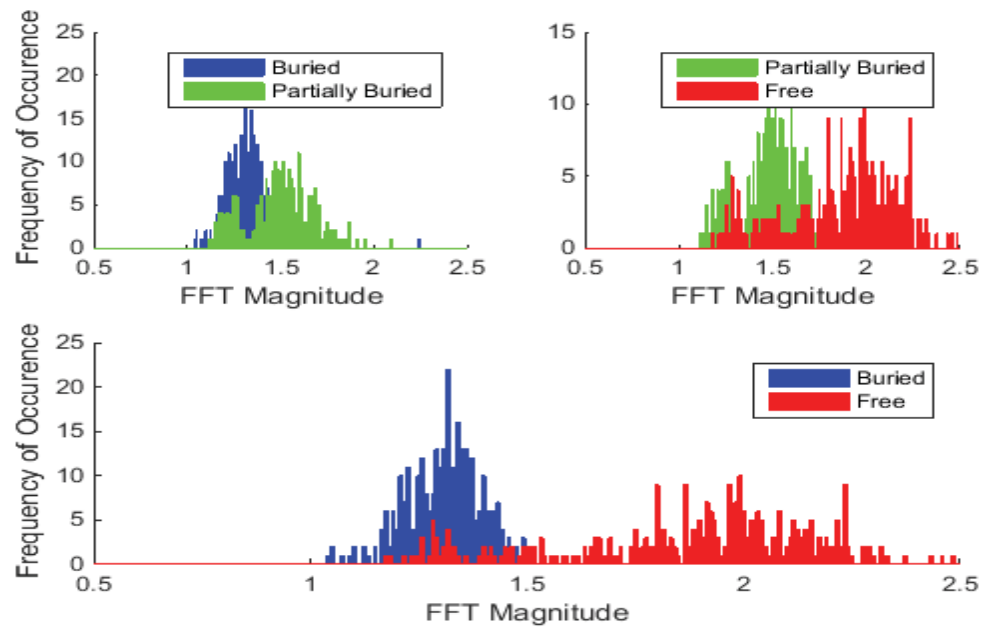

Figure 30. Histogram of Magnitude of FFT for Airfoil Type Sensors at Different States 

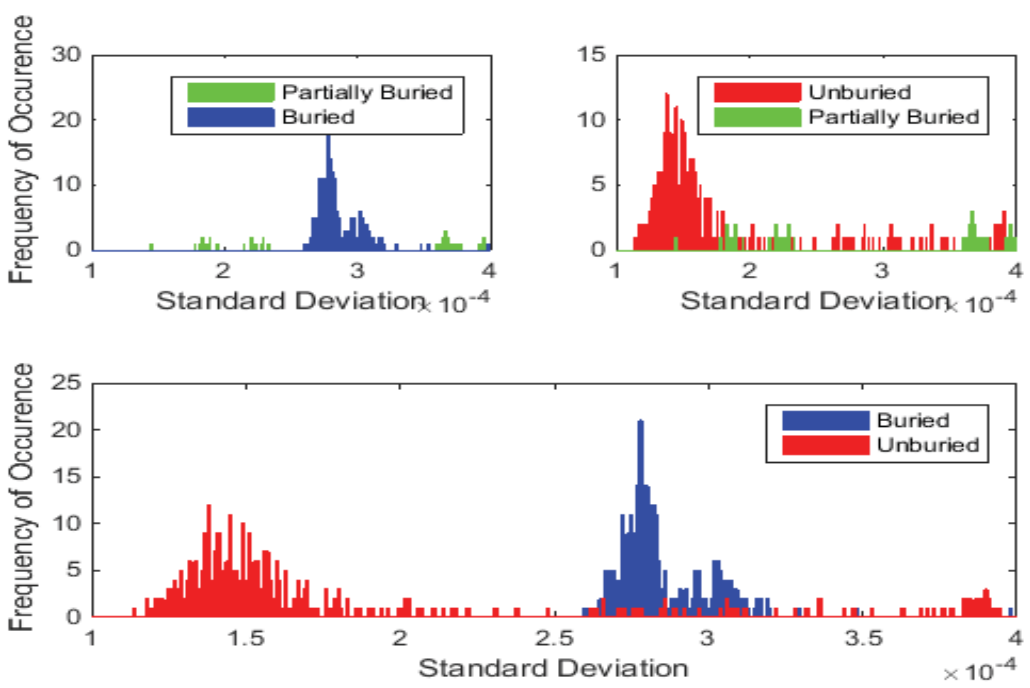

Figure 31. Histogram of Standard Deviation for Whisker Type Sensors at Different States
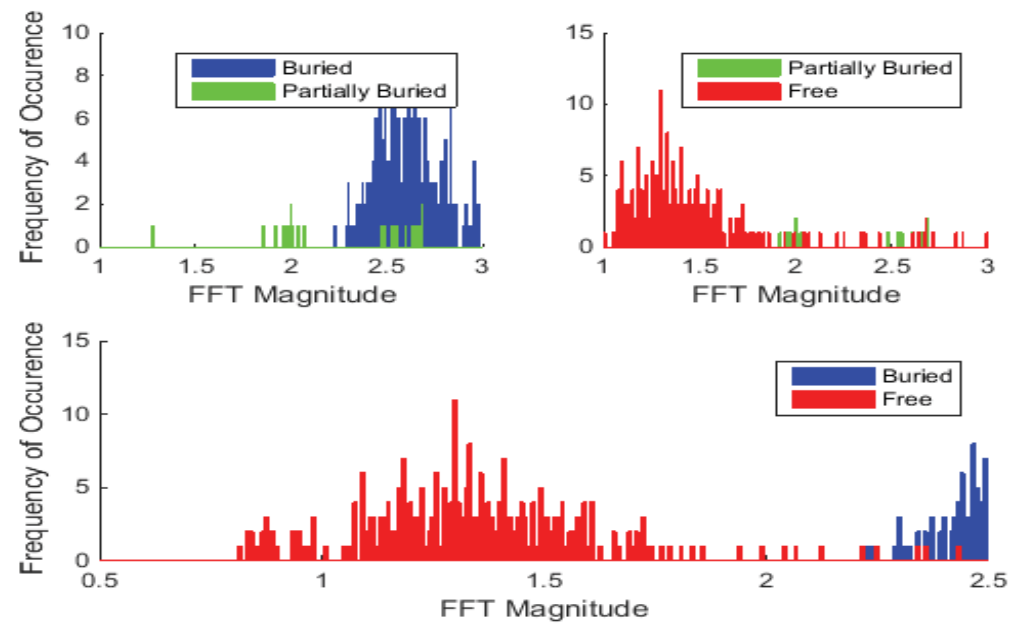

Figure 32. Histogram of Magnitude of FFT for Whisker Type Sensors at Different States

Figure 29 and Figure 30 show the histogram plots of standard deviation and magnitude of FFT for airfoil type sensors and Figure 31 and Figure 32 show the histogram plots of standard deviation and magnitude of FFT for whisker type 
sensors. The magnitude of FFT and Naïve Bayes classifier was used to classify the different states of the sensors.

\section{Naïve Bayes Classifier}

A well-known and relatively easy to implement classification method is used to discern between static and dynamic signals returned by the whisker transducers, namely the naive Bayes classifier. The structure of the naive Bayes classifier is a Directed Acyclic Graph (DAG) that represents the conditional probabilities between variables (Figure 33). In Figure 33, a class variable $\mathrm{C}$ is connected to the feature variables $\mathrm{X}_{1} ; \ldots . ; \mathrm{X}_{\mathrm{n}}$ representing conditional probabilities $\mathrm{P}\left(\mathrm{X}_{\mathrm{i}} \mid \mathrm{C}\right)$ [25]. An important part of the naive Bayes algorithm is probability estimation. Histograms were used for estimating the probabilities for Naive Bayes classifiers and a binning approach was used for the histograms. The histograms give an estimate of the data distribution of the feature measurements made for each class over the training intervals, which are needed for naive Bayes. 


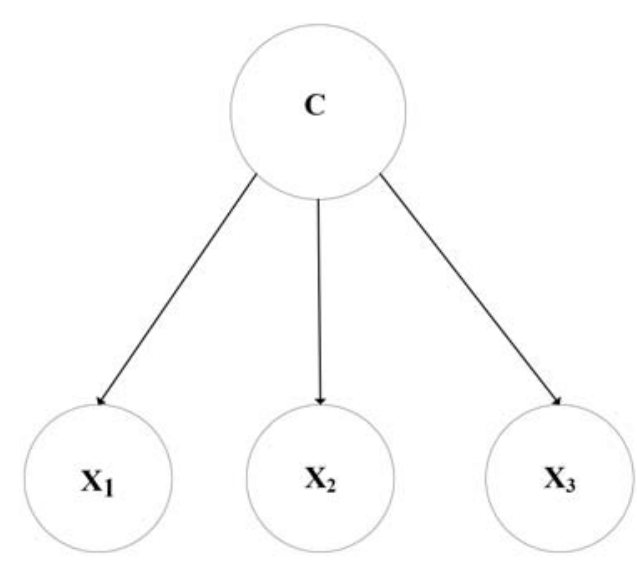

Figure 33. Structure of Naive Bayes Classifier

A naive Bayes classifier assumes that the value of a particular feature of a class is unrelated to the value of any other feature [26]. The joint probability density function for the network is given by the following equation.

$$
P(C j \mid \mathrm{X} 1 \ldots \mathrm{Xn})=P(C) \sum_{i=1}^{n} \mathrm{P}(\mathrm{Xi} \mid \mathrm{C}) .
$$

Solving $P(C j \mid \mathrm{X} 1 \ldots \mathrm{Xn})$ gives the probability for the discrete class variable $\mathrm{C}$ to be in state $\mathrm{j}$.

\section{Training Data}

A training data set is selected from the laboratory data to build up a model, based on the naïve Bayes classifier of the transducer outputs. A different set of laboratory data was set aside and used to validate the model. The data runs included in the training set were not included in the validation set. Usually a dataset is divided into a training set and a validation set in each iteration, in our case two different experiment data sets from the laboratory testing were used as training and test data set. In training runs, 3500 samples from each class were used to train the classifiers. 


\section{Estimation of Classifier}

The posterior probability for each class (dynamic, static, and partially buried) was calculated and was used to estimate the Naïve Bayes classifier using Matlab. Figure 34 and Figure 35 show the Naïve Bayes classifier defined using the training data set for airfoil type sensors for static and dynamic conditions using standard deviation and magnitude of FFT features. Similarly, Bayes classifier was defined for whisker type sensors for different states. The performance of the defined classifiers for different sensor type was tested using the validation data set and was found to be promising to differentiate between the buried and unburied conditions with partially buried conditions returning very similar features to those of the unburied condition.

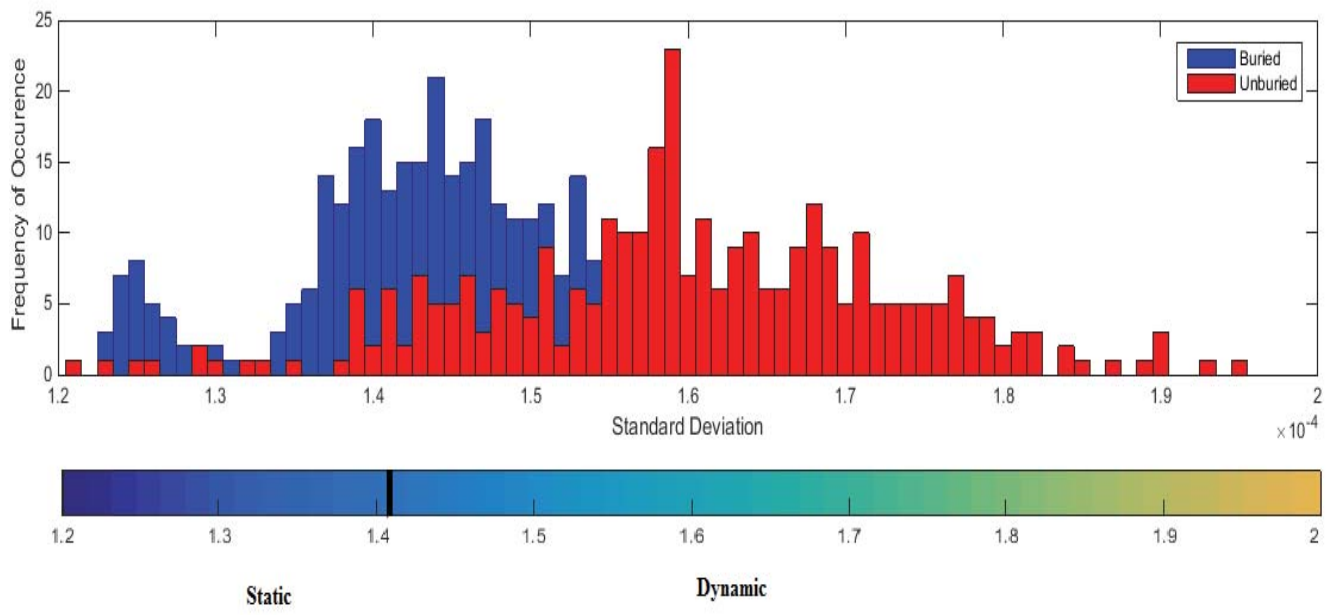

Figure 34. Bayes Classifier for Airfoil Sensor Using Standard Deviation 

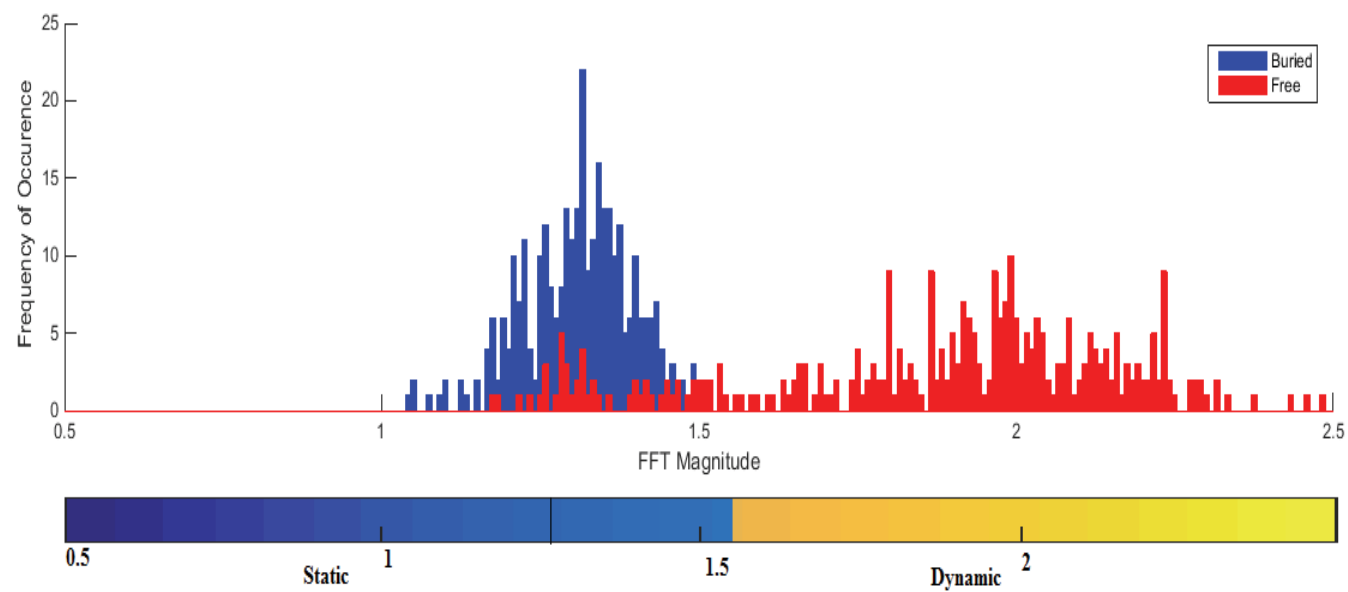

Figure 35. Bayes classifier for Airfoil Sensor Using Magnitude of FFT

\section{Decision file and application of thresholds}

A decision file ALGOTEST is created by the base station at the end of every scour monitoring test. The file contains the time the test was conducted, the unit numbers of the sensing units used, and the states of each transducer. Table 5 shows the states defined. The decision on whether the sensor is dynamic or static is made after comparing the mean, standard deviation and the magnitude of the frequency-domain analysis to a certain pre-assigned. These thresholds were determined for each transducer type after carefully studying the data collected in the laboratory tests. Decision integers are assigned to each sensor, which informs the user about the state of the sensor. Figure 36 shows an example of the decision file created. In addition, sensor fault states including excessive noise and inconclusive (intermediary) flow states are also defined. 
Table 5 Decision states

\begin{tabular}{|c|c|}
\hline Decision Integers & State \\
\hline 0 & Static State \\
\hline 2 & Dilatory State \\
\hline 3 & Transitional State \\
\hline 4 & Dynamic State \\
\hline 5 & Noise \\
\hline
\end{tabular}

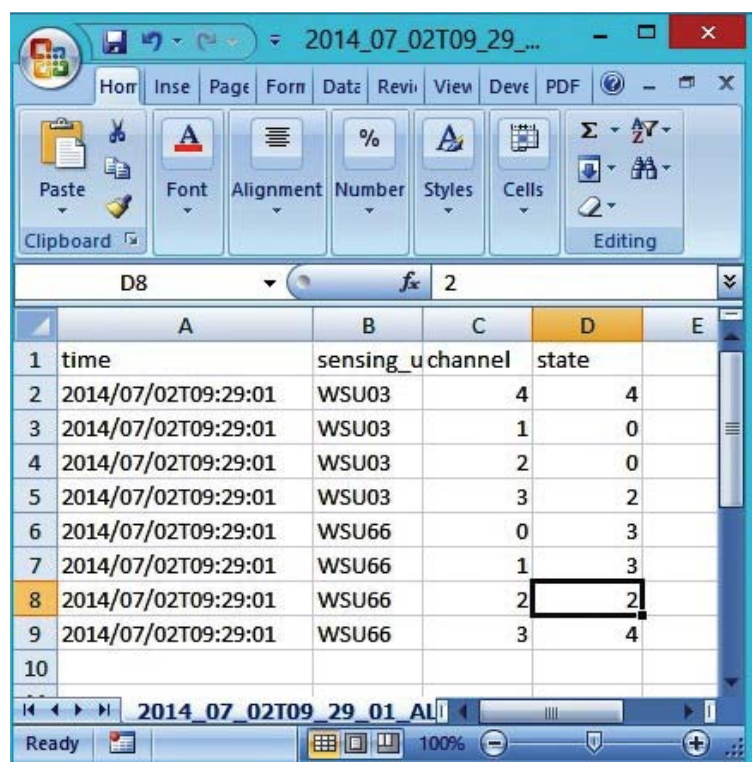

Figure 36. Decision File (Image Source Microsoft Excel, See Appendix-A for Permissions) 
This data is uploaded to the remote decision support client to be interpreted and presented to the end user. A summary of the entire test algorithm is depicted in Figure 37.

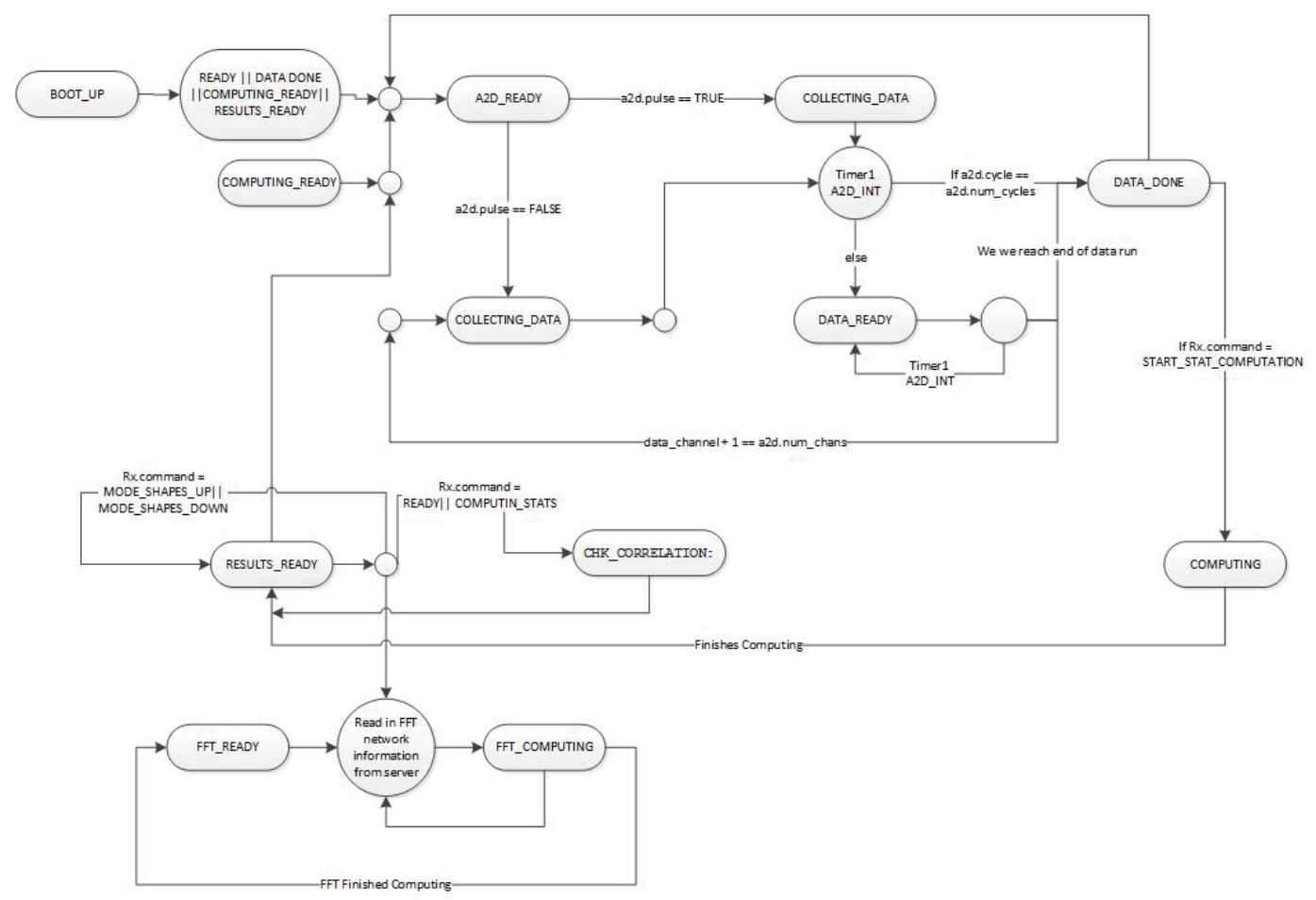

Figure 37. Flow Chart Depicting Narada WSU Algorithm in Scour Sensing Post 


\section{Laboratory Testing}

\section{Introduction}

To confirm the capability of the system, a robust program of validation has been conducted to define the limits of the approach in the laboratory. The proof-of concept laboratory experiments were conducted to validate the ability of the system to monitor and measure scour and to check the ability of the network of wireless sensors to successfully process data. The results of the laboratory validation experiments have been presented here. The tests conducted were all clear water tests.

The laboratory experiments are conducted under a controlled environment which allows a simulation of scour conditions at various water velocities and acts as a test of the scour detection and monitoring system. Scour primarily affects the foundation, abutments, and piers of the bridge. The effect of scour on abutments and piers was studied in the laboratory experiments. During the laboratory experiments, the water velocity was varied to develop scour. The laboratory experiments were performed in the Civil Engineering Hydrology Laboratory located at Michigan Technological University.

\section{Objectives}

Under two laboratory conditions, a flat river bed and a 45-degree river-bank, the proof-of-concept scour detection arrays were studied to meet the following objectives:

- Understand the role flow and scour rates play in the ability of the scour detection arrays to detect scour. 
- Quantify which flow conditions create false indications based on low flow conditions.

- Evaluate new scour monitoring technology under study and identify improvements.

- Explore possible problems that might be encountered in field.

- Obtain a library of signals collected in a controlled environment to correlate outputs to transduce condition (e.g., static vs dynamic, or nominal sensor condition vs fault condition).

- Calibrate thresholds for various transducer geometries to classify signals.

- Test autonomous data collection and interrogation systems for the field validation study and assess the scour prediction method.

\section{Laboratory Facility}

\section{Flume (Water channel)}

The water channel in the hydrodynamics lab is a rectangular, cement tank. The inner walls were coated with epoxy spray to allow for a non-leaking tank. The dimensions of the flume were an inside width of $0.92 \mathrm{~m}$, a total height of $1.04 \mathrm{~m}$, and an inside length of $10.18 \mathrm{~m}$. It consisted of an inlet and outlet at the head and end of the tank, respectively. Water was moved from the outlet to the inlet through a $0.245 \mathrm{~m}$ diameter pipe. The pipe had $271.588 \mathrm{~mm}$ diameter holes facing in the downward position, which allowed water to empty into the head tank. There was a wall that separated the inlet area and the main experimental tank. This internal wall was constructed of $20 \mathrm{~mm}$ diameter PVC pipes, which were stacked parallel to allow for flow straightening into the main water channel. 


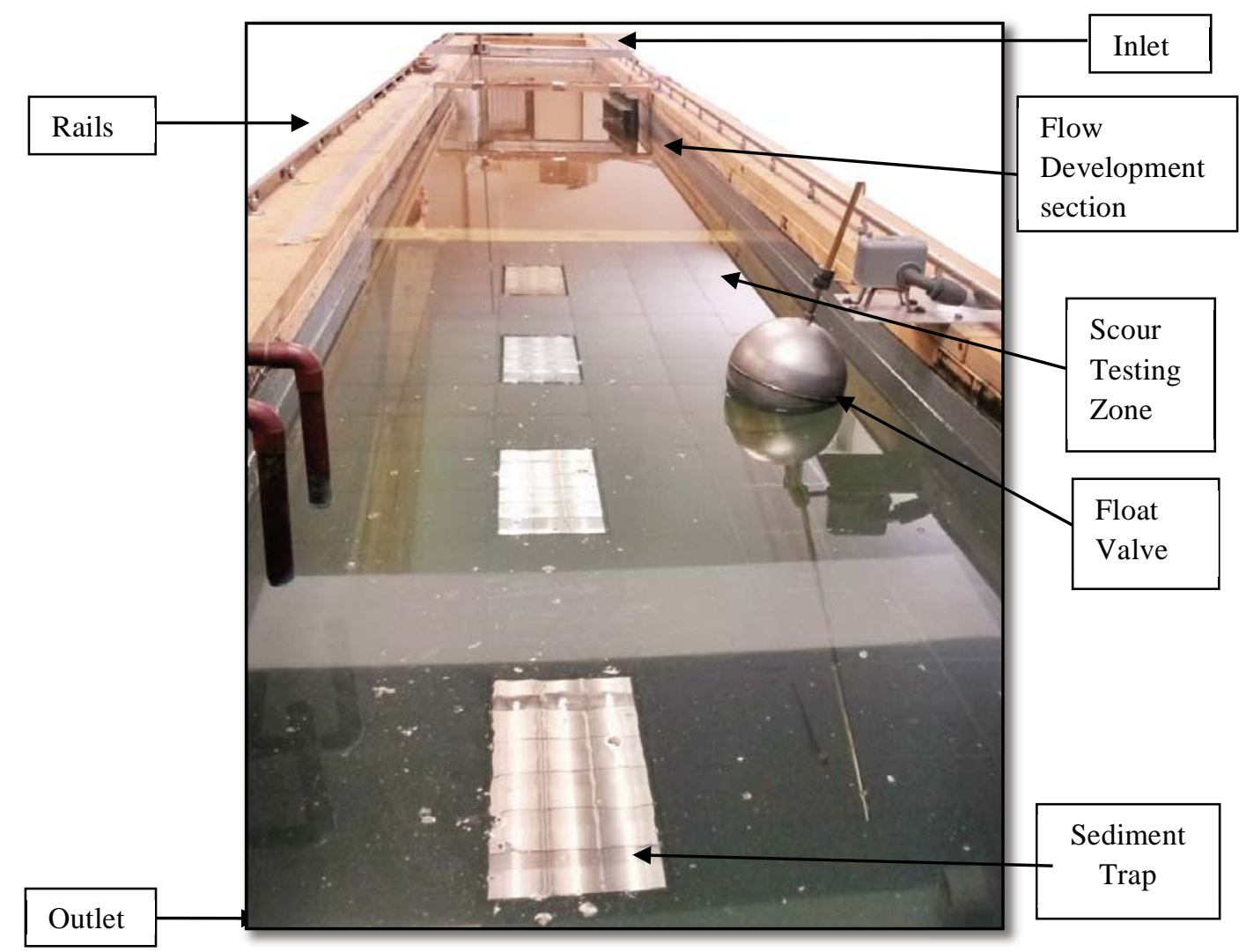

Figure 38. Flume Structure (Image by Author)

\section{Water channel setup for first experiment}

For the first experiment, a flat bed was constructed following the flow straighteners section. This approach area was approximately $1 \mathrm{~m}$ in height and $3 \mathrm{~m}$ in length. This bed was made of plywood and had an epoxy varnish overlay for waterproofing purposes. Soil was then placed on top of the flat bed. The next area was the scour section, which was filled with fine sand. The sand was filled from the bottom of the water channel, level with the soil in the preceding section. The bottom of the water channel was the cement floor of the laboratory. A wooden wall standing $630 \mathrm{~mm}$ tall from the flume floor was constructed near the end of the scour chamber for sediment trapping. The sand, in the scour chamber, was level with the soil in the flat bed section and ended at the wooden wall. The water height was made to be just above the top sensor of the pier. 


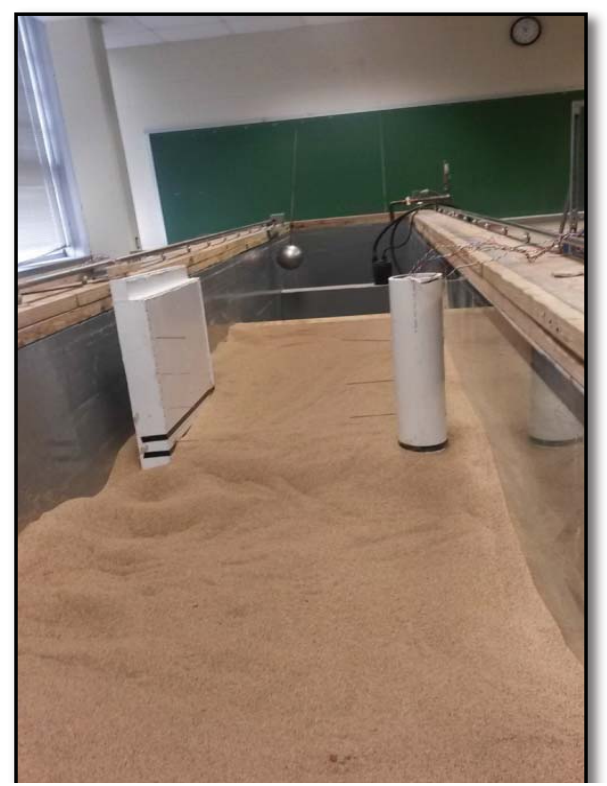

Figure 39. Flume Setup for First Experiment (Image by Author)

\section{Water channel setup for second experiment}

For the second experiment, the flat bed was removed. It was replaced with an angled embankment bed that was positioned 45 degrees from floor of the flume. The flow straighteners were repositioned to accommodate for the embankment slant. The approach area had dimensions of $3 \mathrm{~m}$ length and $0.5 \mathrm{~m}$ height. It was constructed of plywood and had a coarse sand paper overlay. The scour section was filled with the same fine sand from the first case. The sand was angled at a 45 degree position, which lined up with the bed. This exposed a portion of the flume floor from the angled bed section through the scour section. The wooden standing wall was removed and the scour chamber tapered off before it reached the sediment trap area. 


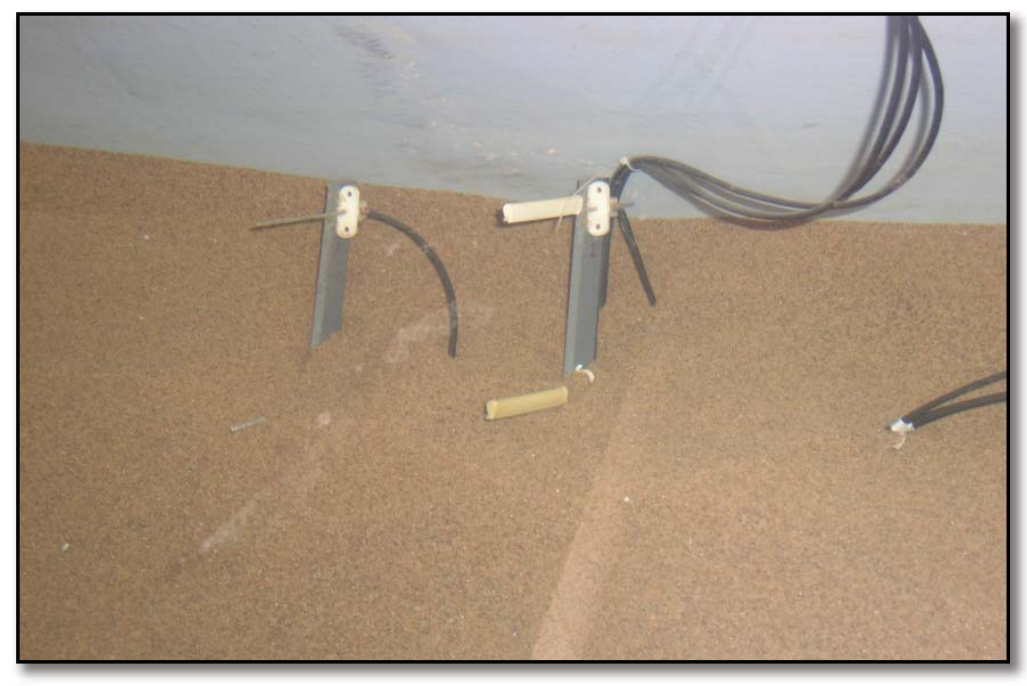

Figure 40. Flume Setup for Second Experiment (Image by Author)

\section{Scour experiment structure}

\section{Model Pier and Abutment}

To conduct the proof-of concept laboratory experiment, a pier and an abutment were assembled using Poly vinyl chloride (PVC) board and pipes for the laboratory test. The pier was made using a $0.10 \mathrm{~m}$ diameter PVC pipe attached to a box made up of a rigid form of PVC board. Also, a simple abutment was constructed using PVC boards and $0.05 \mathrm{~m}$ PVC pipe was used to make piles. Waterproof glue and quick setting super glue were used to hold the assembly together.

The dimensions of the pier and abutment were to scale, with the flume being used for the experiment. The models were made to closely match a similar structure in the field. The total height of the pier was $0.86 \mathrm{~m}$ and it was made to be $0.10 \mathrm{~m}$ wide and the abutment was approximately $0.86 \mathrm{~m}$ in height and $0.71 \mathrm{~m}$ wide. Both the pier and abutment were coated with waterproofing silicon and were spray painted white to make them waterproof and weather proof. The transducers were attached to the pier and abutment assembly for the experiment. Holes that fit the sensors were 
drilled into the abutment and sensors were mounted on the models. The hole was then coated using silicon for waterproofing. Four whisker type transducers each were mounted on both pier and abutment models. The transducers were spaced $0.152 \mathrm{~m}$ from each other. Figure 41 shows the model pier and abutment used for the laboratory experiment.
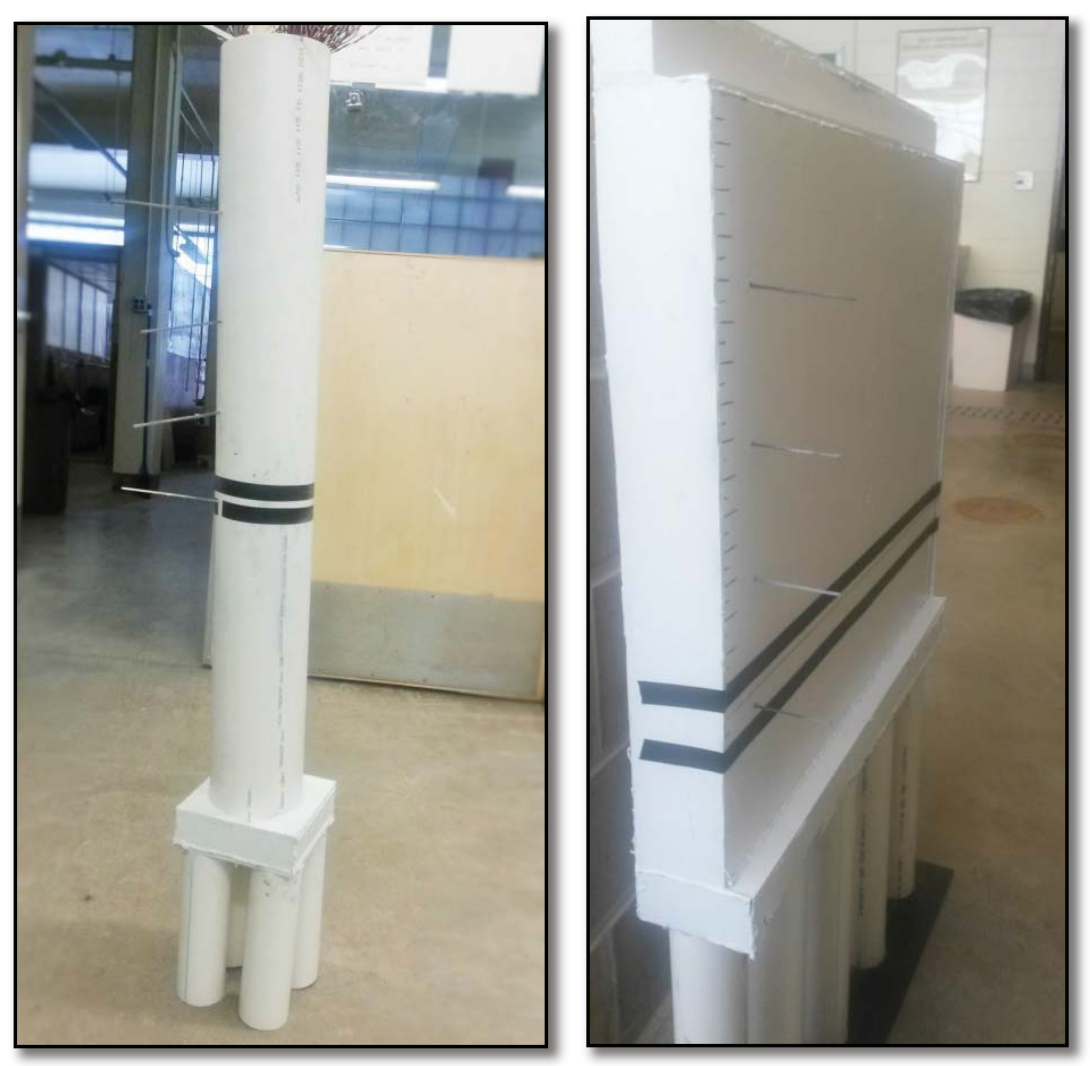

Figure 41. Pier and Abutment (Image by Author) 


\section{Posts}

For the river bank experiment, two simple posts, one with bio-inspired whisker type sensors and the other with airfoil sensors were made. PVC strips $0.61 \mathrm{~m}$ in length and $0.02 \mathrm{~m}$ in width were used as posts. The sensors were mounted on the posts using waterproof glue. Three sensors each were mounted $0.07 \mathrm{~m}$ apart in each post. Figure 42 shows an example of the post used.

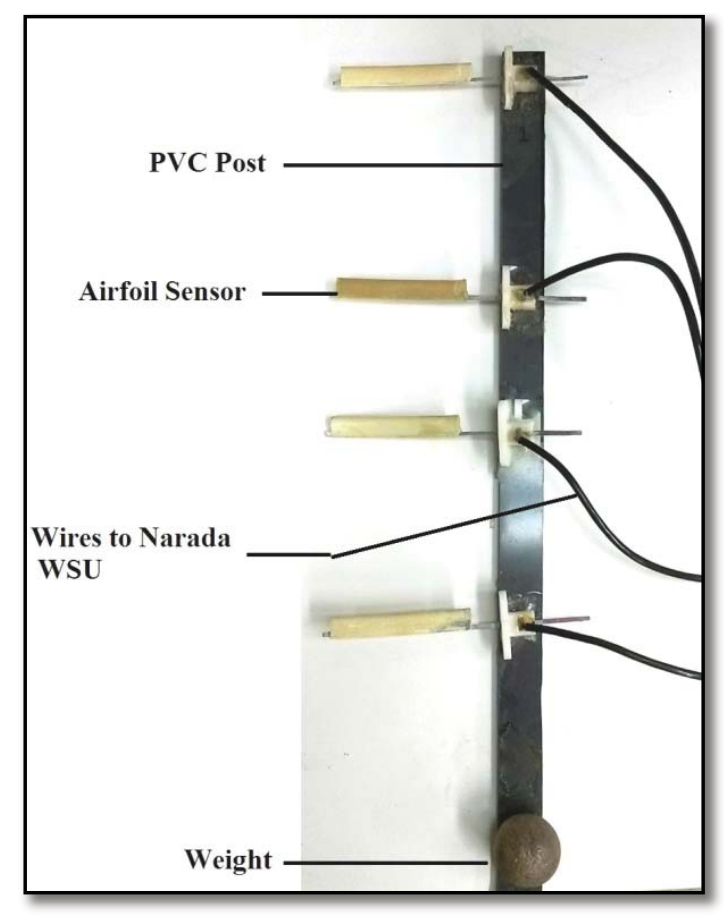

Figure 42. Sample Airfoil Posts (Image by Author) 


\section{Experimental Setup}

\section{Pier and abutment experiment}

In the first case, the flat bed was filled with a layer of soil. There were three divisions of soil types laid on the flat bed. The first section was about $1 \mathrm{~m}$ in length and was filled with gravel about $25.4 \mathrm{~mm}$ deep. This gravel section transitioned into a gravel/sand mixture of about $25.4 \mathrm{~mm}$ deep and $1 \mathrm{~m}$ in length. The final transition was filled with fine sand to about $25.4 \mathrm{~mm}$ deep, spilling into the scour sector. The sand had $\mathrm{a} \mathrm{d}_{50}$ of $0.56 \mathrm{~mm}$. The same sand was filled to this height in the scour section of the flume. Placed in the sand was a replica of a bridge pier and abutment. These models were scaled 1/30th of the size of a bridge pier and abutment. The pier was made of a $30 \mathrm{~mm}$ PVC tube and was placed perpendicular to the flow and channel bed. It had a height of $0.3 \mathrm{~m}$ above the sand bed. The abutment was placed in contact with the east wall of the flume and the pier was placed $0.30 \mathrm{~m}$ away from the west wall of the flume. The distance between the abutment and pier was $0.46 \mathrm{~m}$. The height of the abutment above the sand bed was $0.3 \mathrm{~m}$. It had a width of $20 \mathrm{~mm}$ and a length of $40 \mathrm{~mm}$. The width of the abutment faced the oncoming flow, while the length of the abutment was parallel to flow. For laboratory testing purposes and to make the installation easy, the bio-inspired whisker type sensors were mounted on the model abutment and pier itself for scour detection and monitoring. Four sensors each were mounted on both the pier and abutment models. For the purpose of lab validation tests, two wireless sensing unit were used. The transducers mounted on the model pier and abutment was connected to each wireless sensing unit. Power was provided to the wireless sensing unit using an AA battery pack. In this test the Narada wireless sensors were setup to send the data to a laptop computer for analysis. 


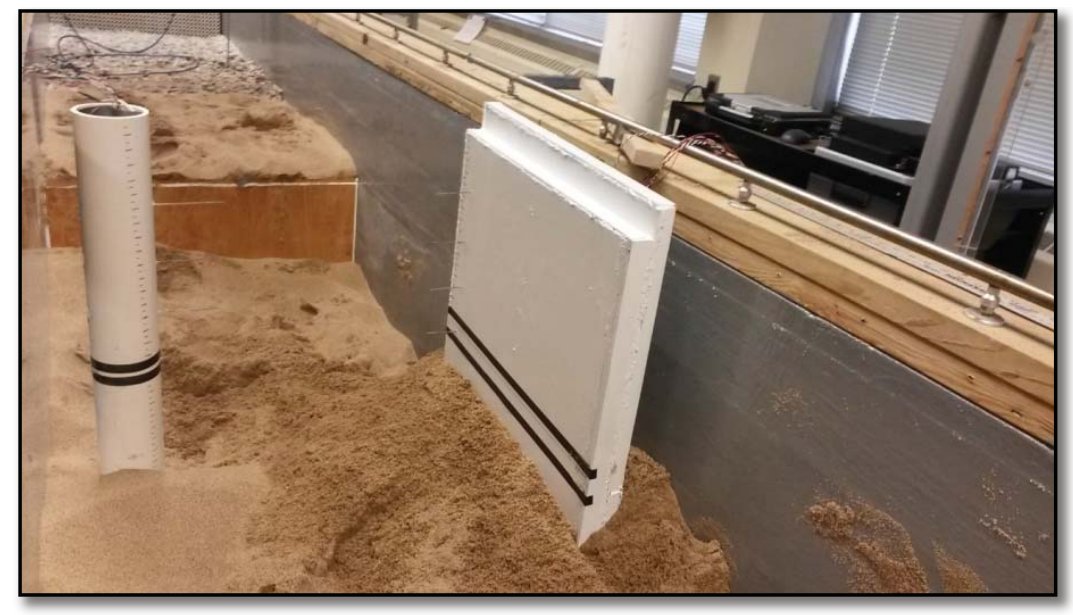

Figure 43. Flume Setup for Pier and Abutment Experiment (Image by Author)

\section{River bank experiment}

For the second case, two posts were used. The flume was setup to match a river bank for this experiment. The flow development zone, which was a wooden structure from the previous setup was removed and replaced by an inclined wooden structure to create a riverbank. Clean, fine sand was used to create a river bank on one side of the flume. The posts were then buried in the river bank so that the sensors were aligned perpendicular to the flow of water. To make the posts stable, weights were attached to the ends of the posts. The transducer on the top of the post was left unburied, the bottom two transducers were fully buried in the sand and one of the transducer was partially buried when placed in the flume. For the purpose of lab validation tests, WSU were used. The transducers that were mounted on the posts were connected to the wireless sensing unit. Power was provided to the WSU using an AA battery pack. Similar to the previous setup, the Narada wireless sensors was setup to send the data to a laptop computer for analysis. 


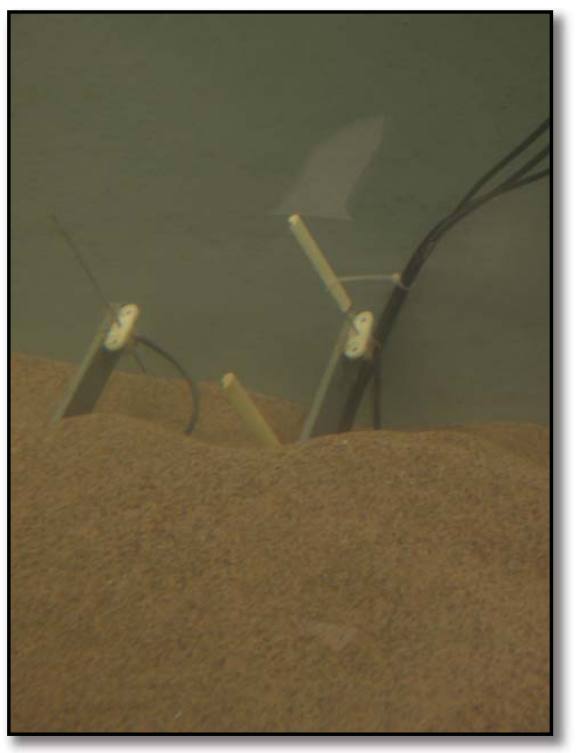

Figure 44. River Bank Experiment (Image by Author)

\section{Experimental Procedure}

In an effort to understand the impacts of flow and scour on the ability of the detection sensor to detect scour, a model of a bridge pier and abutment were installed in a laboratory flume. In both laboratory set-ups, the proof-of-concept sensors were tested and associated scour was measured.

In order to generate a database of signals to be used for classification (static versus dynamic signals), multiple pump speeds were used. Pump speeds started at 9.06 hertz, and were increased in increments of 1.0 hertz, up to a maximum pump speed of 30 hertz.

Scour was measured for each pump speed at multiple time intervals. Once equilibrium was reached, scour was measured and recorded as "worst scour" for each pump speed. Equilibrium is defined as less than 1\% change in scour over a 3hour period. The worst scour location, either upstream or downstream of each structure, was also documented. 
The data were collected periodically for the entire duration of the experiment using two WSU for detecting scour. The data collected were processed to determine the dynamic and static states of the sensors.

The sand used for the experiment had sediment of mean size, $d_{50}$, of $0.56 \mathrm{~mm}$ $(0.0018 \mathrm{ft})$ and geometric standard deviation $=\sigma_{\text {sed }},=\left(d_{84} / d_{16}\right)^{1 / 2}=1.25$.

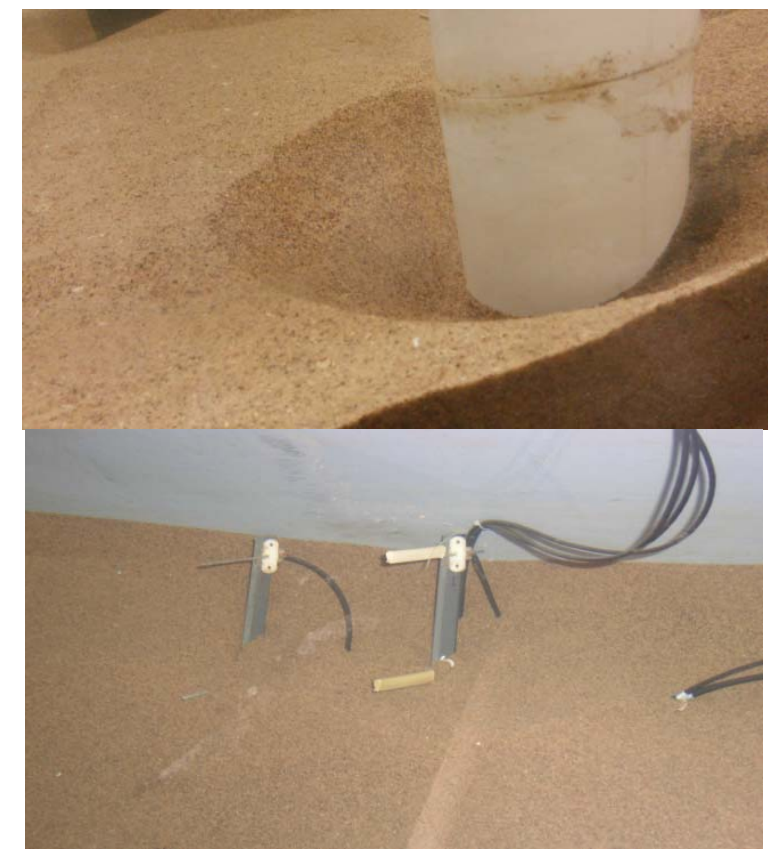

Figure 45. Scour Hole Developed (Image by Author)

The plots in Figure 46 show the depth of scour developed during various velocities during the experiment. The first plot shows the depth of the scour hole generated during the experiment using model pier and abutment. The next figure shows the plot of depth versus time for the river bank experiment. 


\section{Scour Depth Plot Pier and abutment experiment}
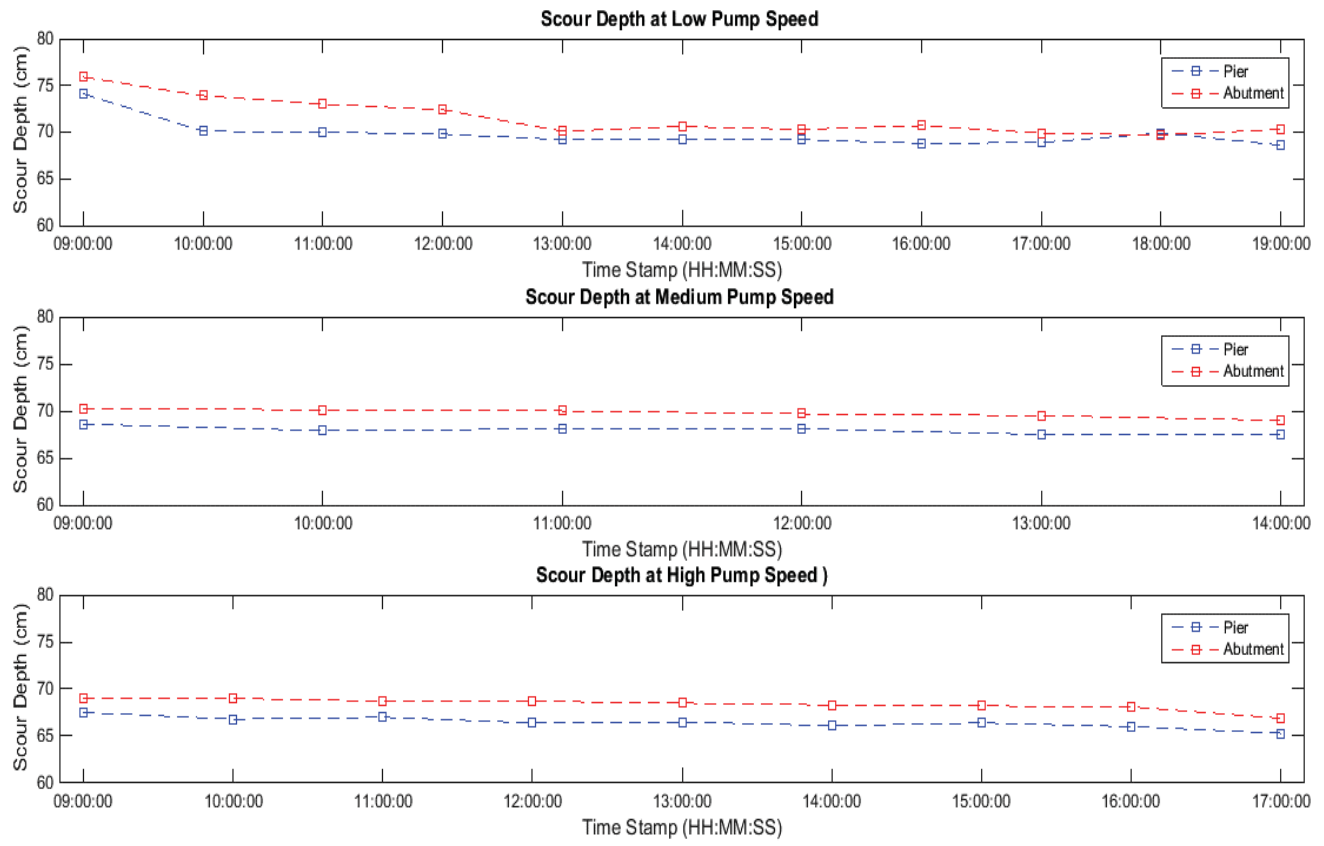

Figure 46. Scour Depth Plot Pier and abutment experiment

The plots above (Figure 46) show the scour hole developed at different velocities for experiments using model pier and abutment. The plots above show that the depth of the scour hole fluctuates over time. It can be seen that as time progresses the depth of the scour hole tends to vary. 


\section{Scour Depth Plot River bank experiment}
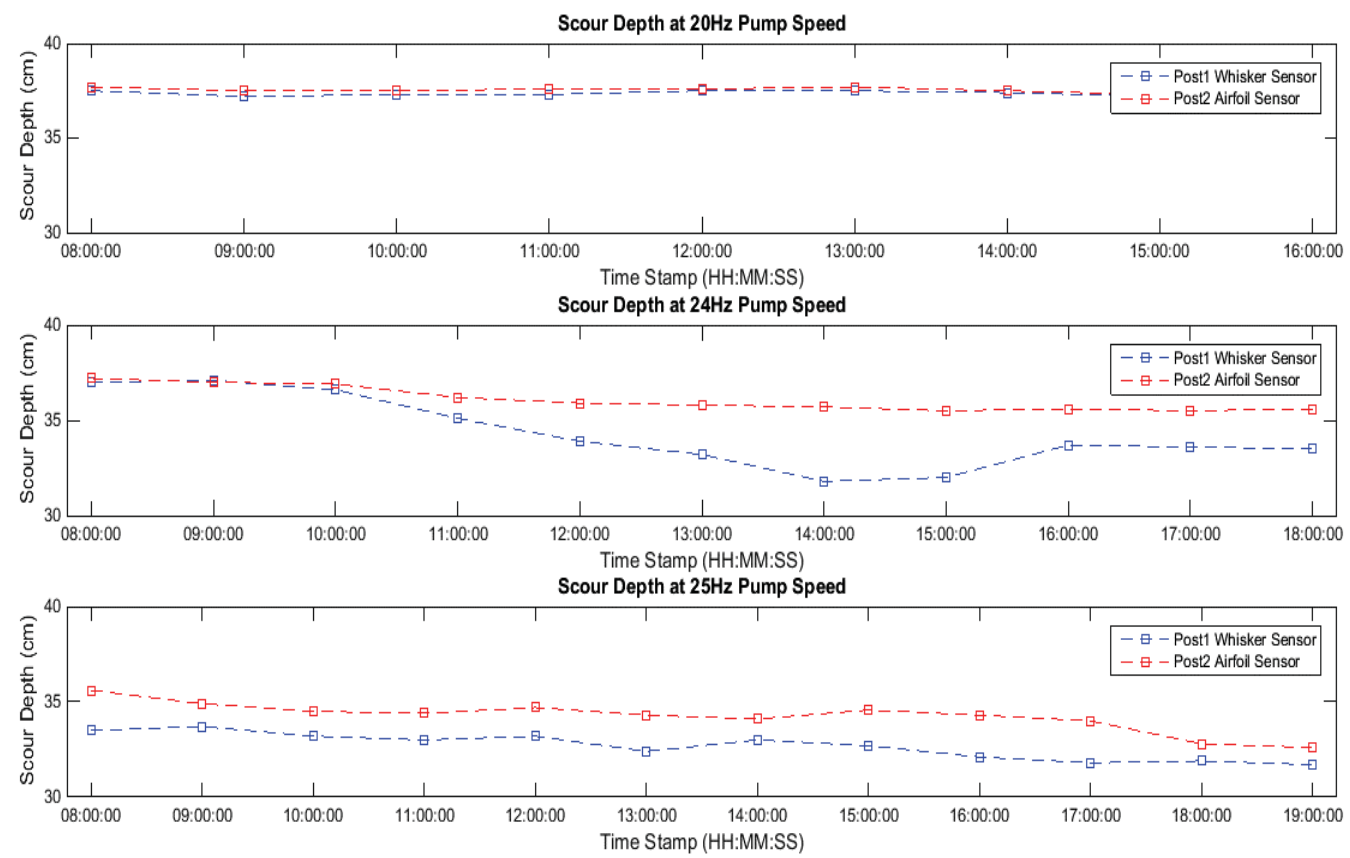

Figure 47. Scour Depth Plot River bank experiment

The plots in Figure 47 show the scour depth developed at different velocities for the river bank experiment. From the above plots it can be seen that for this experiment the depth of the scour hole generated near the posts constantly fluctuates. It can be clearly seen that the depth of the scour hole near post 1 during the beginning of $24 \mathrm{~Hz}$ pump speed slowly increases but then later gradually decreases. 


\section{Results}

Figure 48 and Figure 49 show time domain plots of sensor output and show typical dynamic and static behavior when buried and unburied.

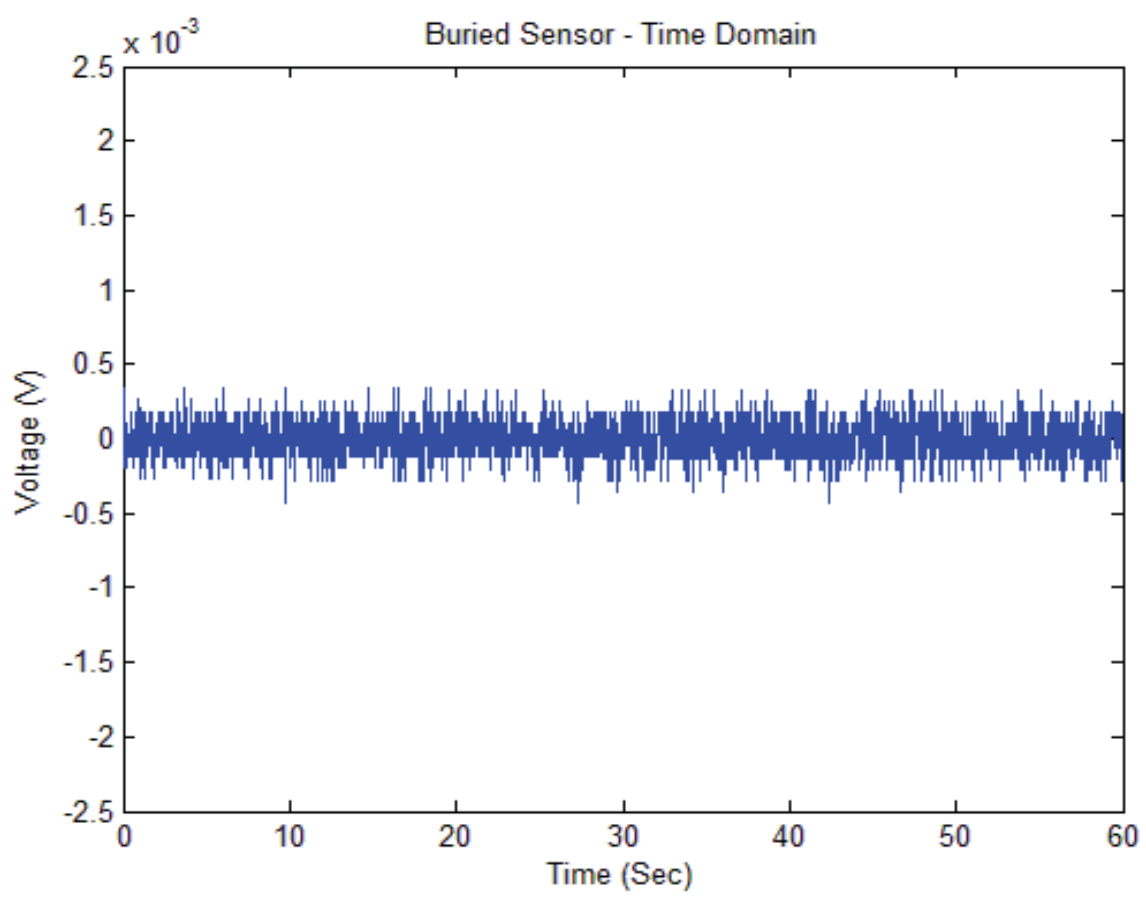

Figure 48. Time Domain Plot of Buried Sensor (Static State) 


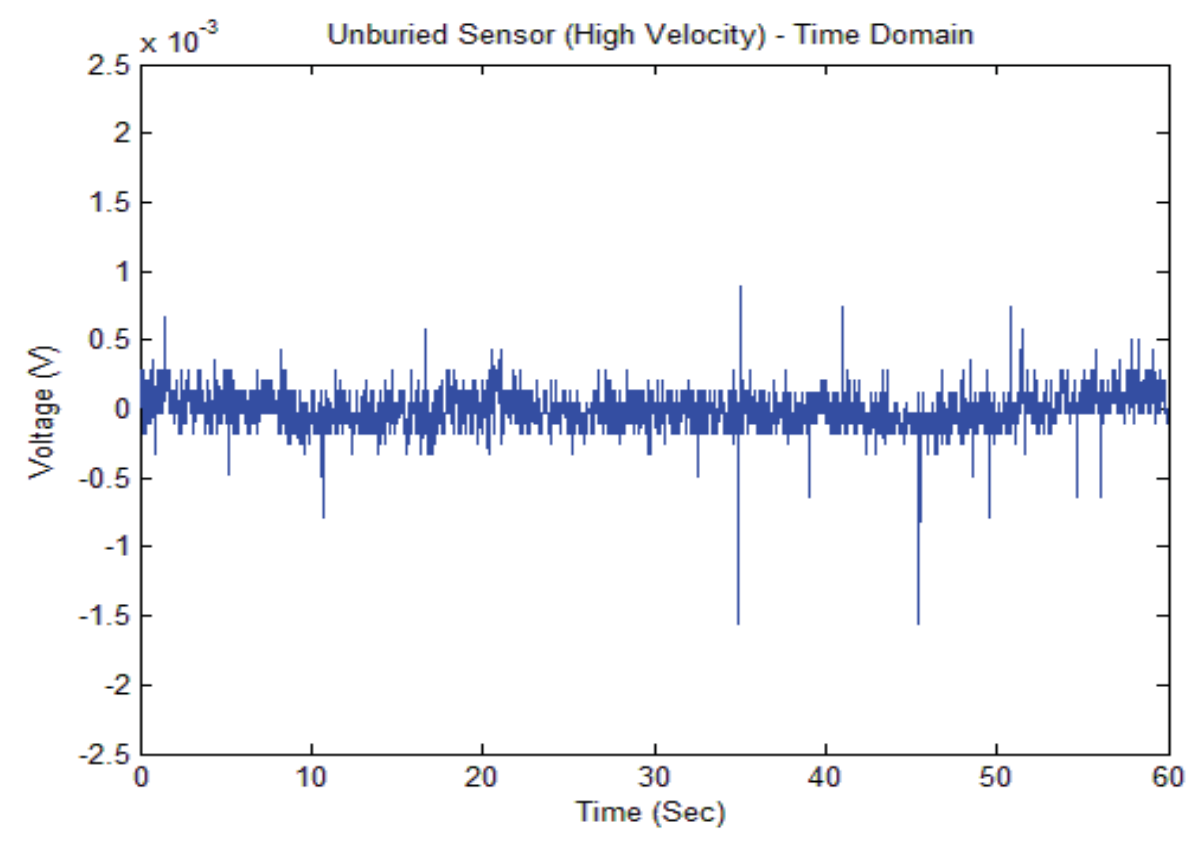

Figure 49. Time Domain Plot of Unburied Senor (Dynamic State)

\section{Data collection analysis of laboratory experiment: model pier and abutment}

In this laboratory experiment the data were collected every hour and transmitted to the server, which in this case was a laptop. The developing scour hole developed was measured at regular intervals. The plots below show the underwater time domain response of the sensors at different flow velocities.

\section{TeST PARAMETERS:}

Sampling frequency $(\mathrm{Hz})=100 \mathrm{~Hz}$

Sampling time $(\mathrm{sec})=30$ Seconds

Samples per polling cycle $=3000$ samples

No of Units $=2$ 
Unit ID (s) = 77 WSU (Whisker) (Abutment)

Channel(s) LEGACY_CH0, LEGACY_CH1, LEGACY_CH2, LEGACY_CH2

Unit ID (s) = 66 WSU (Whisker) (Pier)

Channel(s) LEGACY_CH0, LEGACY_CH1, LEGACY_CH2, LEGACY_CH2

Sample time histories are shown below from Figure 50 to Figure 55.

Time domain plot at low water velocity (Time stamp 12:03:08):

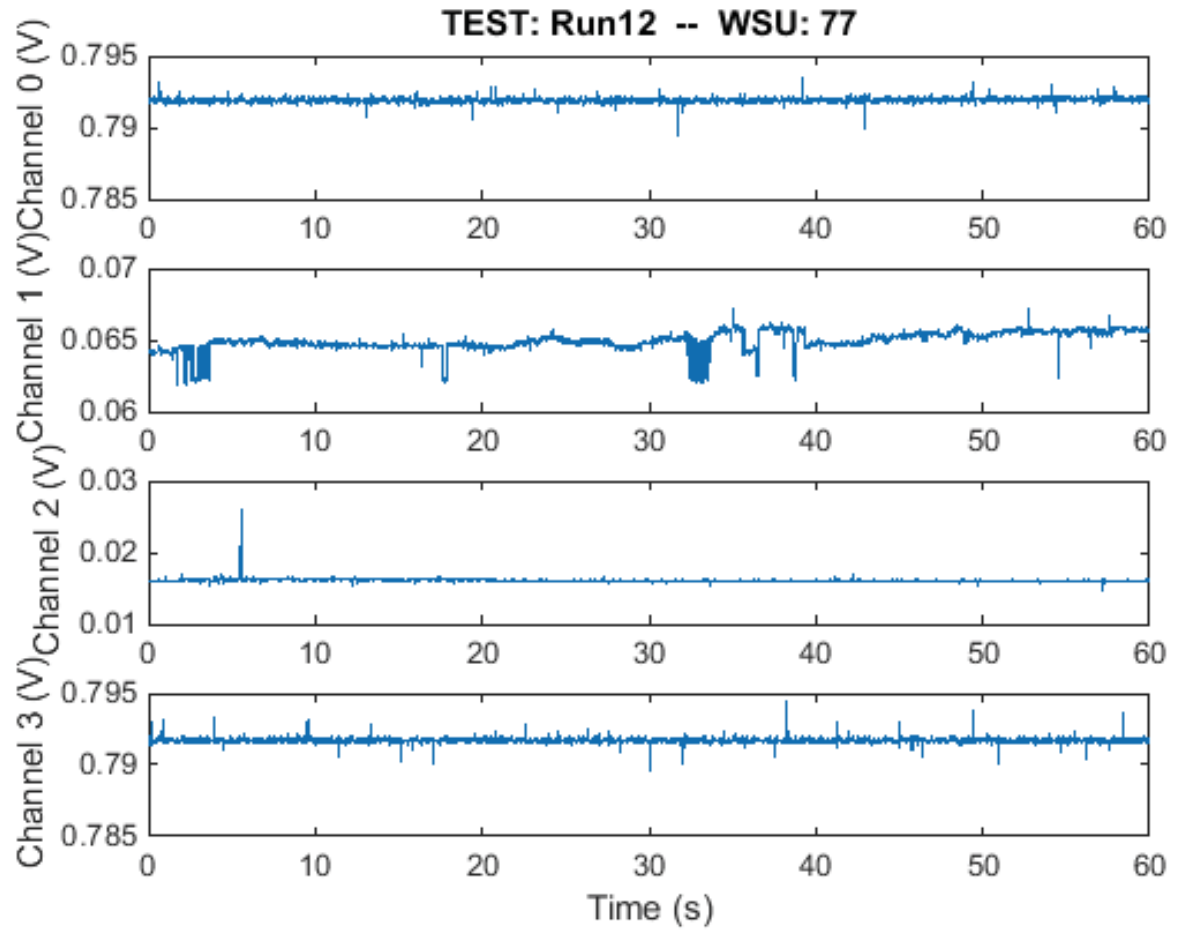

Figure 50. Time Domain Plot at Low Water Velocity for WSU 77 


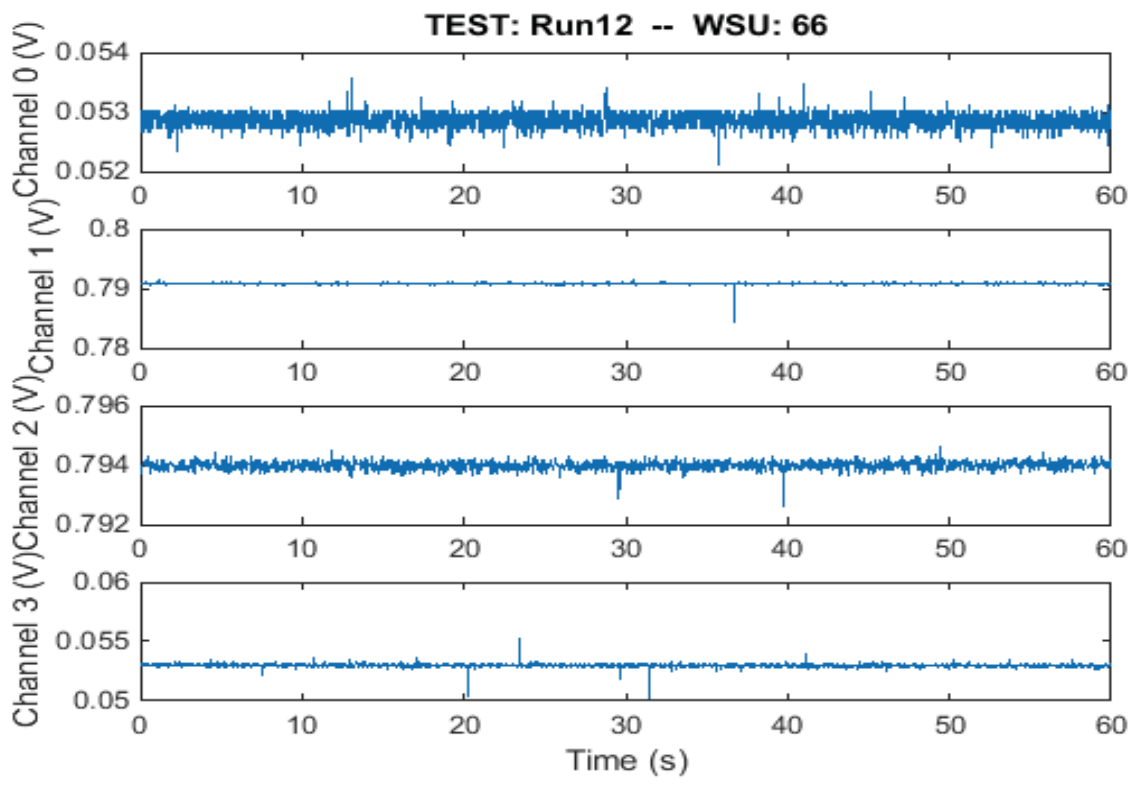

Figure 51. Time Domain Plot at Medium Water Velocity for WSU 66

Here, on Figure 50, Channel 1 in WSU 77, a sensor fault condition was observed. This signal and others like it will be useful to establish a library of fault signals. Channel 0 in both the WSUs show dynamic conditions. The remaining Channels show a static condition of sensors, telling us that they are buried and stationary. 
Time domain plot at medium water velocity (Time stamp 13:29:12):

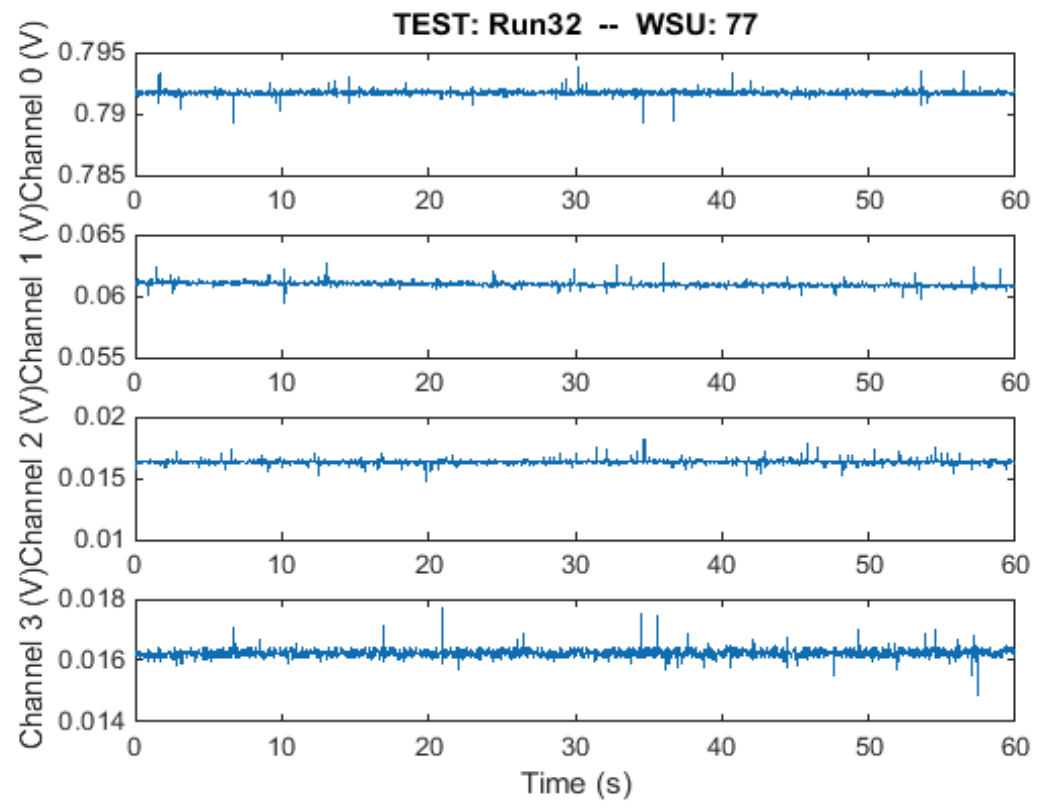

Figure 52. Time Domain Plot at Medium Water Velocity for WSU 77
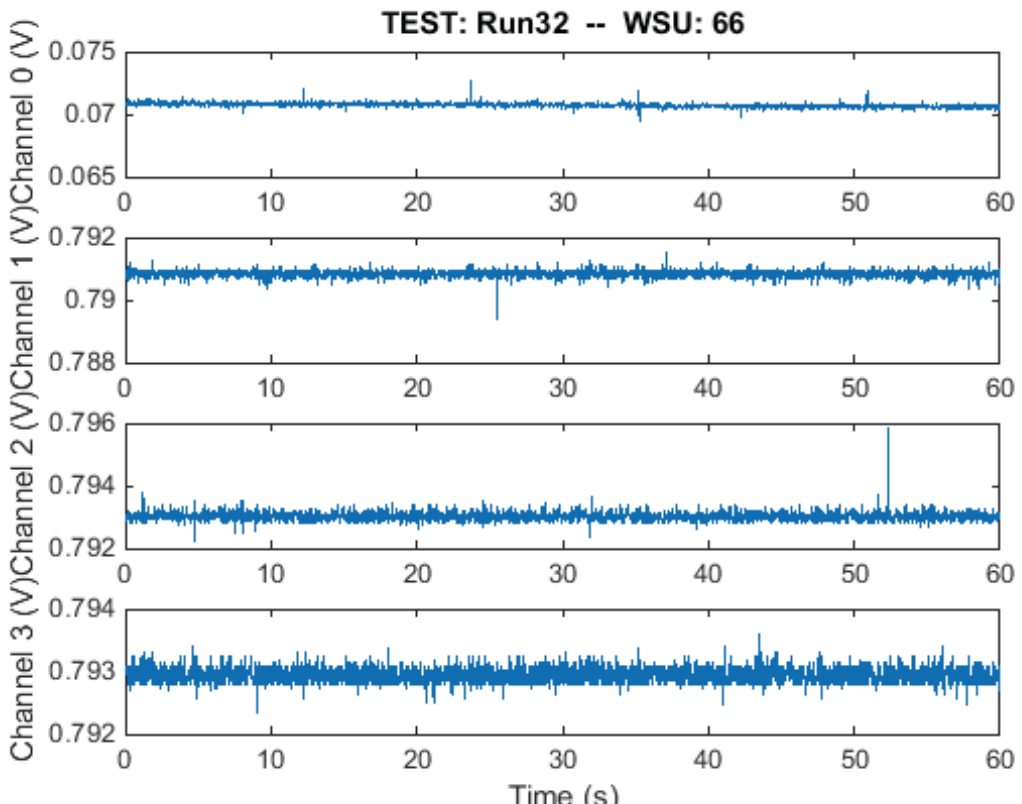

Figure 53. Time Domain Plot at Medium Water Velocity for WSU 66 
Here, on Figure 53, Channel 3 in WSU 66, a sensor measuring noise was observed. Channels 1 and Channel 2 in both the WSU show a partially buried condition of sensors. It has both the characters of dynamic and buried properties.

Time domain plot at high water velocity (Time stamp 15:09:28):

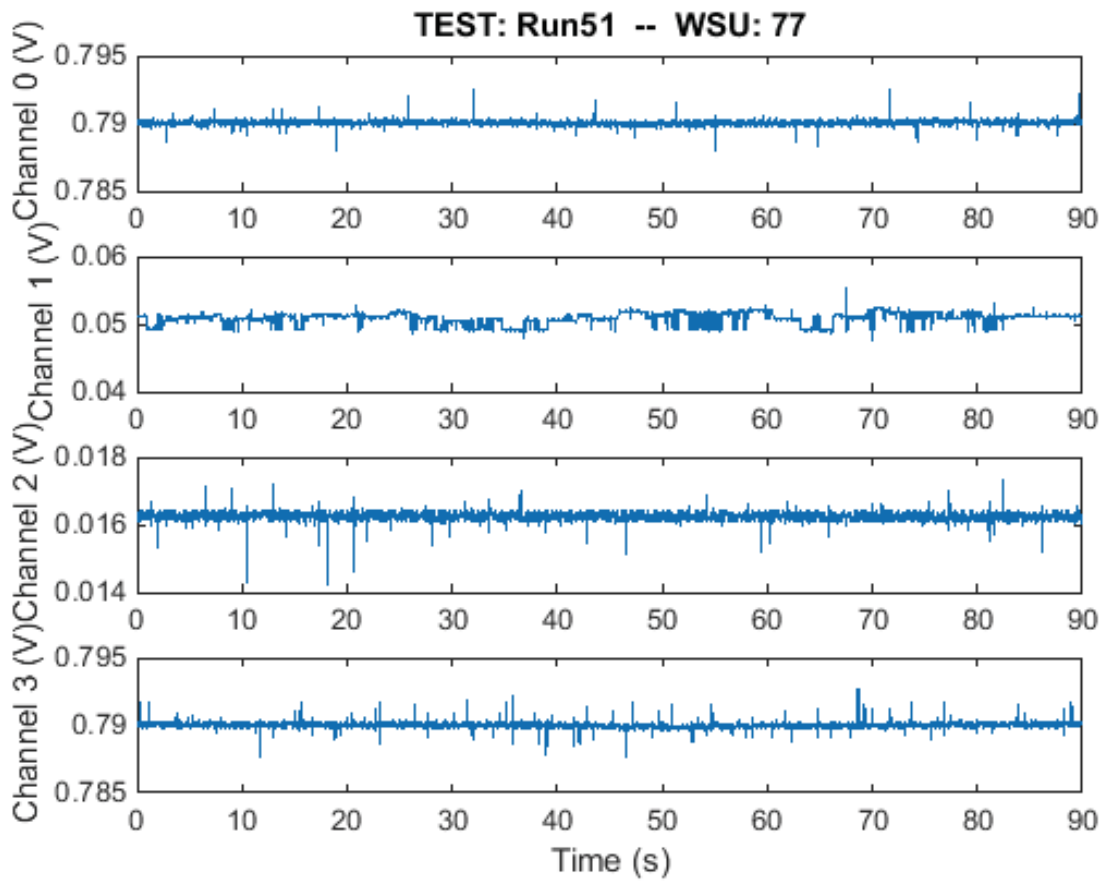

Figure 54. Time Domain Plot at Medium High Velocity for WSU 77

Here, Figure 54, on Channel 1 poor connectivity of the sensor to the data acquisition devise was observed. Channel 0, Channel 2 and Channel 3 show dynamic state, showing that they are unburied and water bound. 


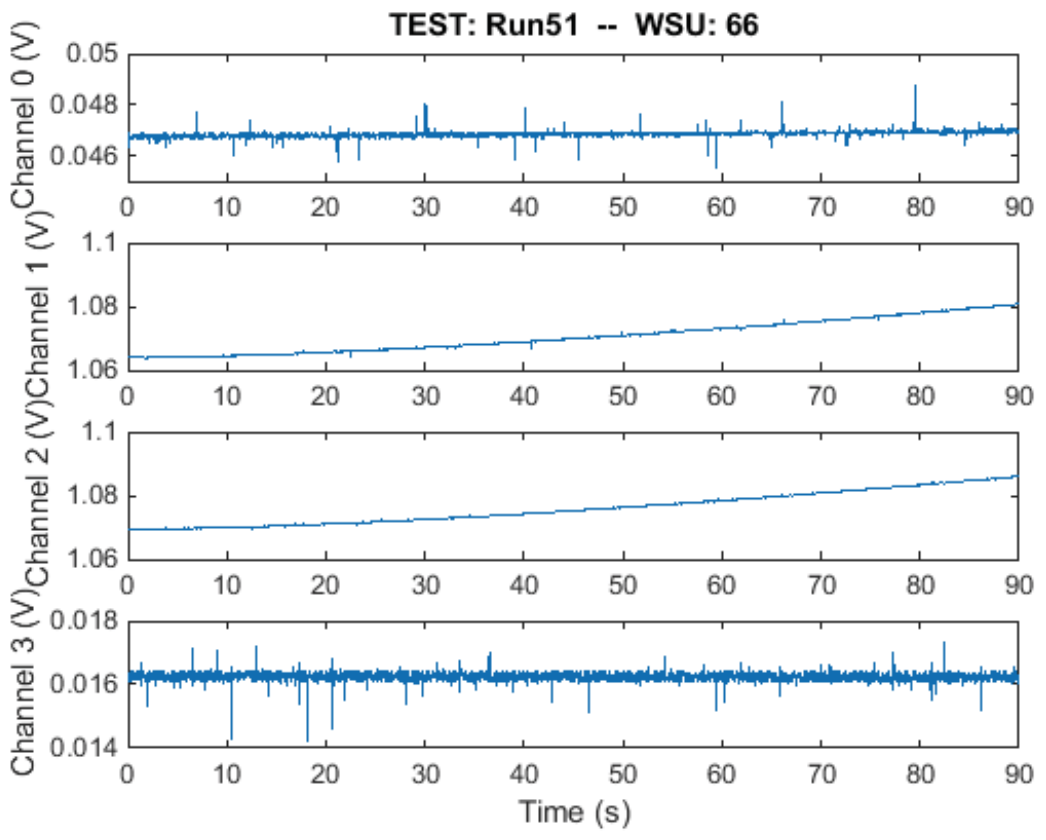

Figure 55. Time Domain Plot at Medium Water Velocity for WSU 66

In some cases, minor mean drift was observed on one or more channels in the outputs from the Hall sensors. In these cases it became clear that the trending algorithms were necessary as part of the autonomous data interrogation algorithms.

\section{Data collection analysis of laboratory experiment: model post}

In this laboratory experiment, the data were collected every hour and transmitted to the server which in this case was the Single board computer. Scour hole developed. The plots below show the underwater time domain response of the sensors at different flow velocities.

Sampling frequency $(\mathrm{Hz})=50 \mathrm{~Hz}$

Sampling time $(\mathrm{sec})=60$ Seconds

Samples per polling cycle $=3000$ samples 
No of Units $=2$

Unit ID (s) = WSU 7 Post 1 (Whisker)

Channel(s) LEGACY_CH0, LEGACY_CH1, LEGACY_CH2

Unit ID (s) = WSU 53 (Airfoil)

Channel(s) LEGACY_CH0, LEGACY_CH1, LEGACY_CH2

Sample time histories are shown below from Figure 56 to Figure 61.

Time domain plot at low water velocity (Time stamp 15:03:32):
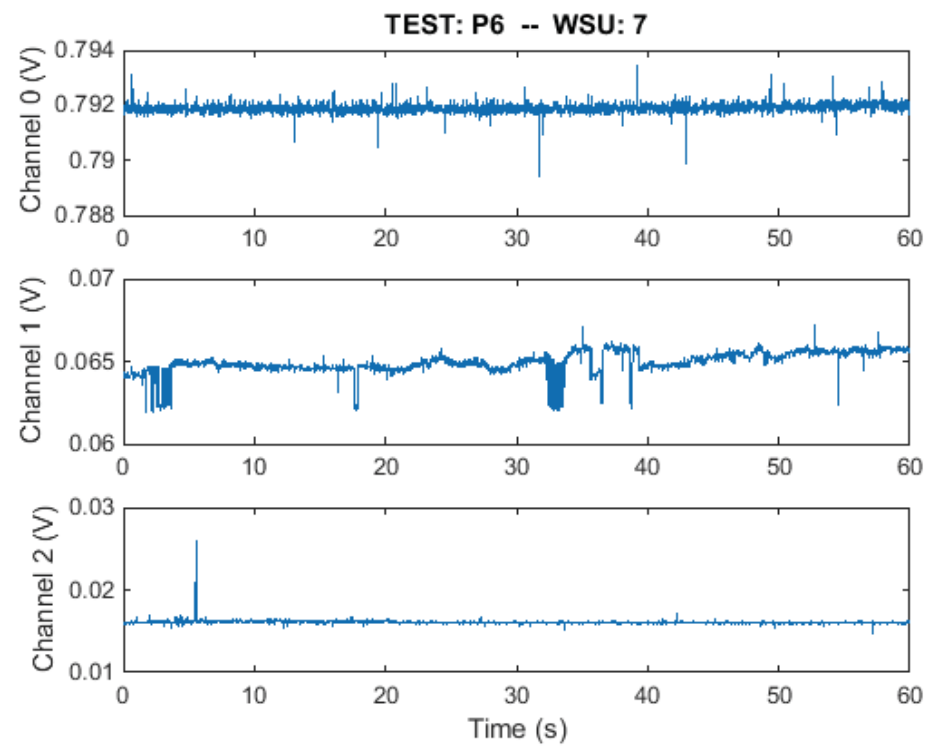

Figure 56. Time Domain Plot at Low Water Velocity for WSU 7

Here, on Figure 56, in Channel 0 a dynamic condition was observed showing that the sensor is unburied. In Channel 1, a sensor fault condition was observed. The fault condition can be caused due to poor connectivity of the sensor to the data acquisition devise. Channel 2 shows static conditions, affirming that it is buried. 

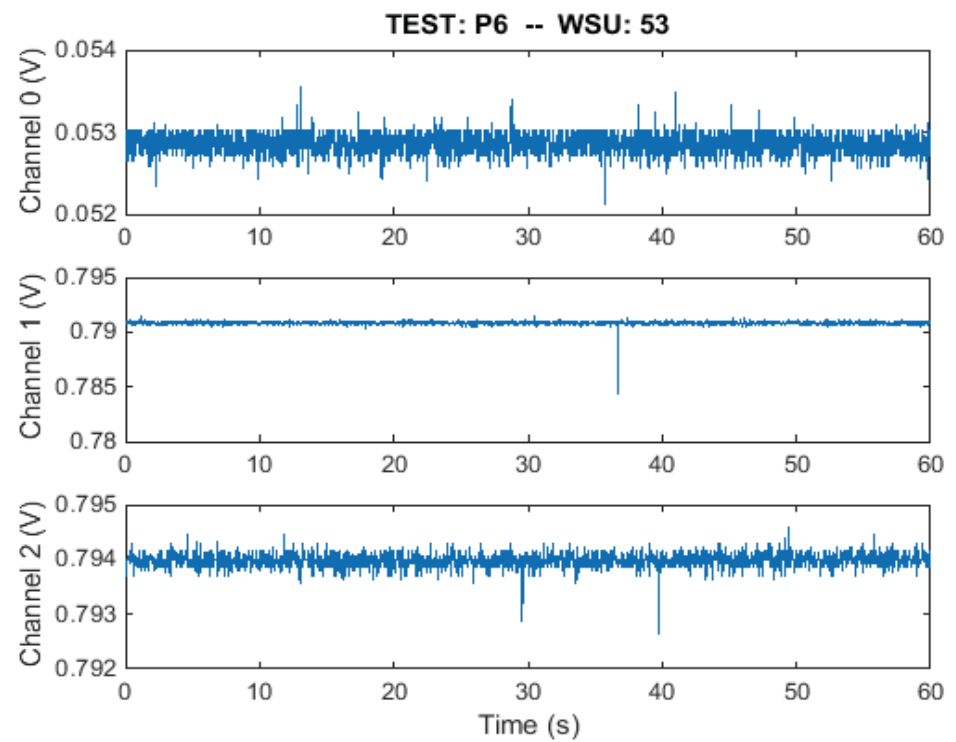

Figure 57. Time Domain Plot at Low Water Velocity for WSU 53

Here, in Figure 57, in Channel 0 a partial dynamic condition was observed showing that the sensor is unburied. Channel 1 shows a static condition and channel 2 shows a sensor measuring only noise. 
Time domain plot at medium water velocity (Time stamp 14:01:25):
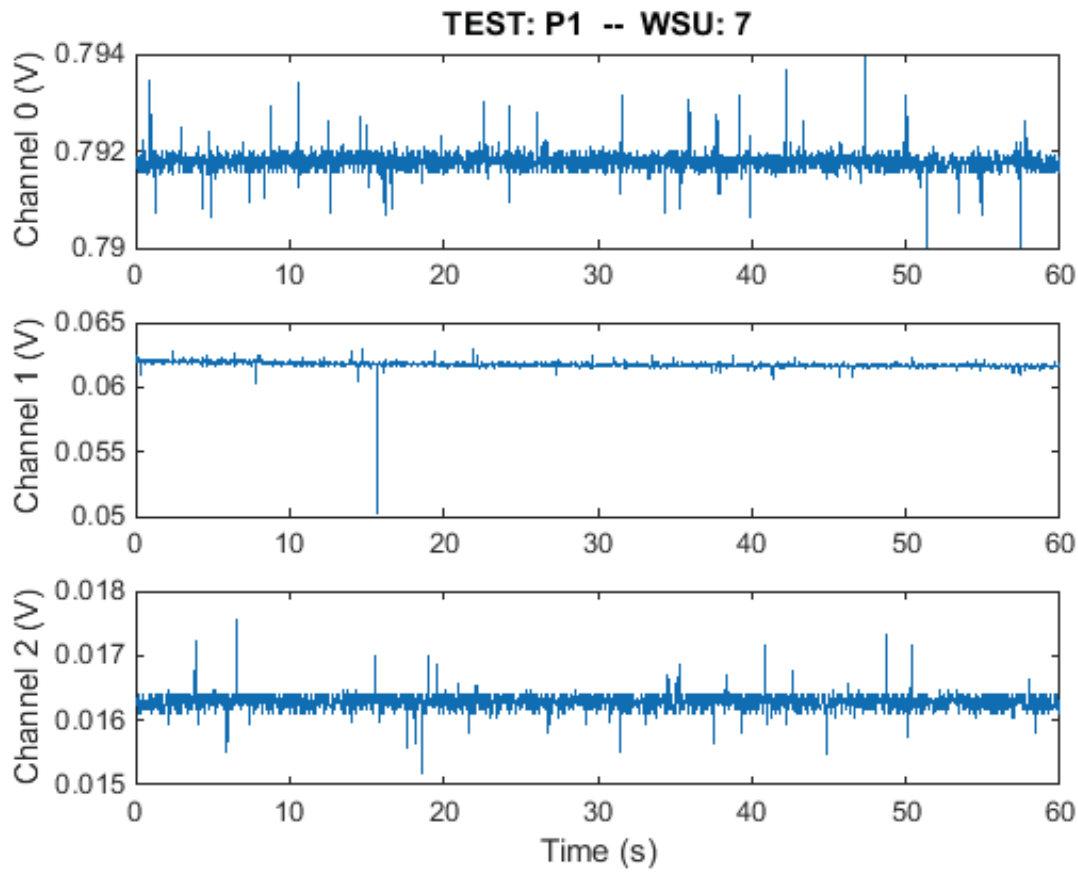

Figure 58. Time Domain Plot at Medium Water Velocity for WSU 7

Here, in Figure 58, in Channel 0, a dynamic condition was observed showing that the sensor is unburied. Channel 1and Channel 2 show a static condition along with noise. 

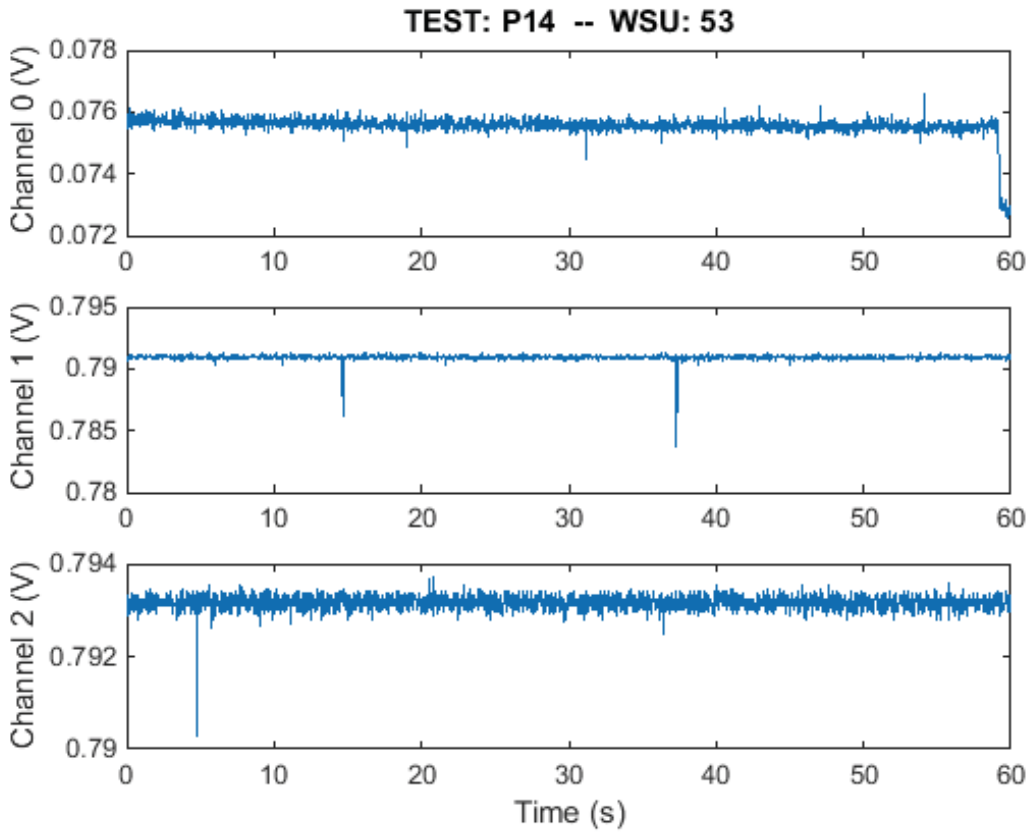

Figure 59. Time Domain Plot at Medium Water Velocity for WSU 53

Channel 1 in Figure 59 shows a sensor measuring dynamic condition and Channel 2 and Channel 3 show a buried condition. 
Time domain plot at medium water velocity (Time stamp 18:06:52):
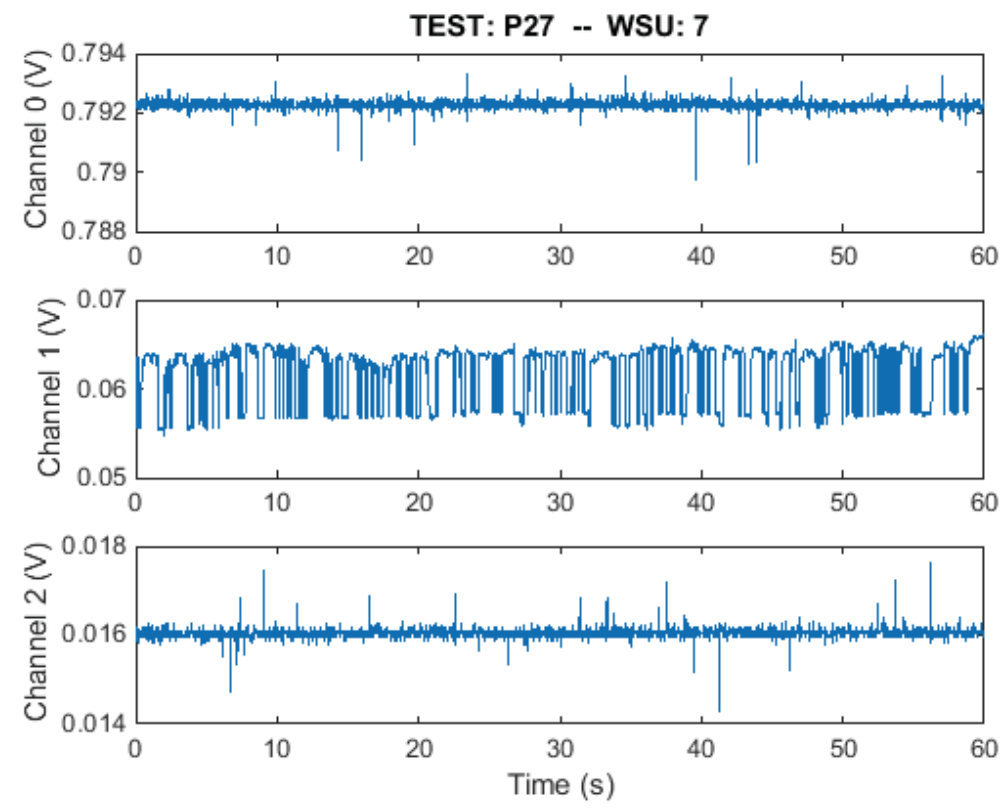

Figure 60. Time Domain Plot at Medium Water Velocity for WSU 7

In Figure 60, Channel 1 shows a sensor fault condition (poor connectivity of the sensor to the data acquisition devise). This signal and others like it will be useful to establish a library of fault signals for a later classification algorithm development. 

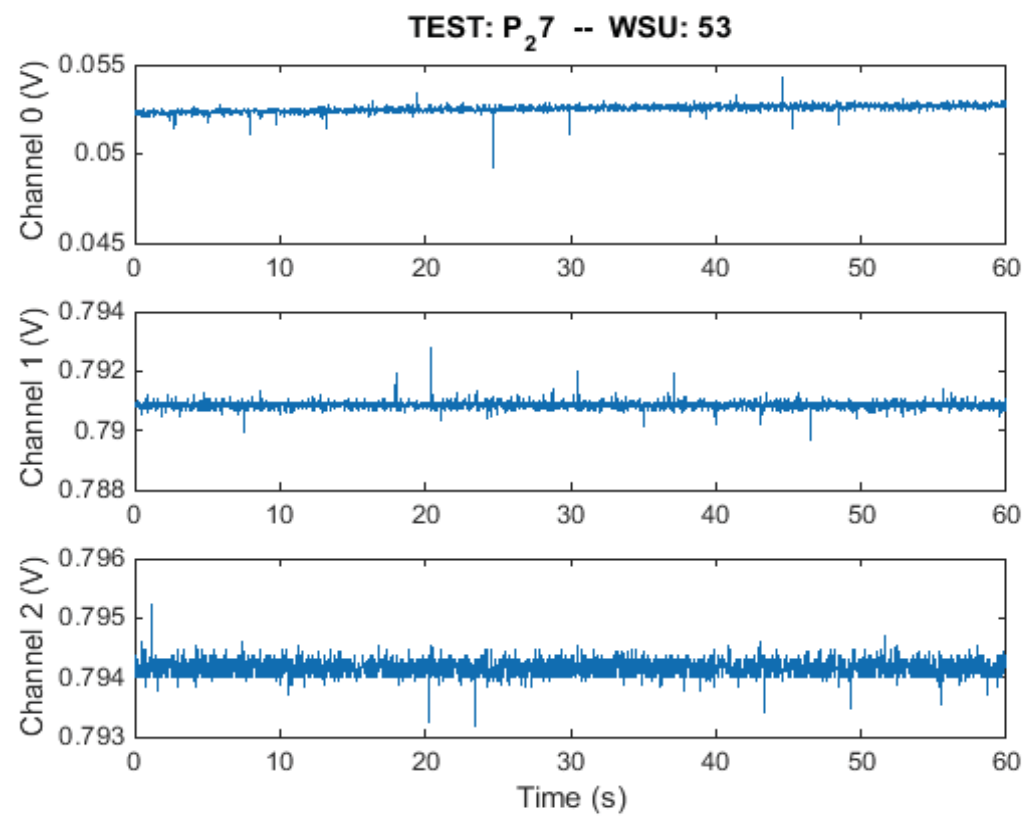

Figure 61. Time Domain Plot at Medium Water Velocity for WSU 53

In Figure 61, dynamic condition was observed in Channel 0 and Channel 1 showing that the sensor is unburied. Channel 2 shows a sensor measuring static condition. 
The plot below in Figure 62 shows the success rate of the sensors (whisker and airfoil type) in correctly stating the state of the sensors at different velocities of water in the flume.

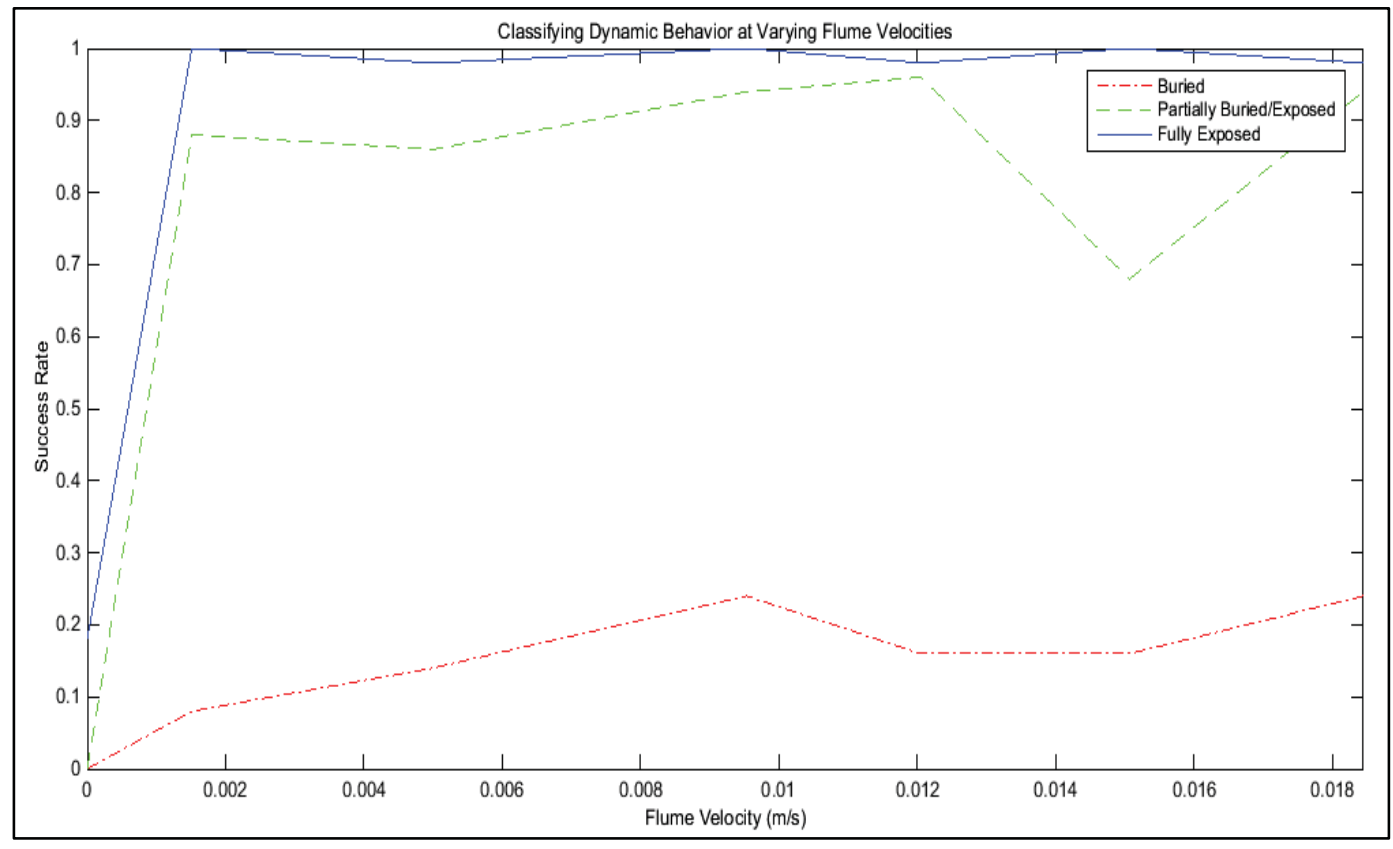

Figure 62. Success Rates of Sensors 


\section{Field Deployment}

\section{Introduction}

This chapter describes the two bridges that were selected as the site for the field implementation and evaluation of the selected in-situ monitoring system. It also describes the instrumentation that was developed to make installation of scour sensor elements easy and practical. It goes on to describe the procedure that was undertaken for the installation of the monitoring system at the selected site for validating the installation of the system.

\section{Selected Bridge for Field Implementation and Evaluation}

\section{Pilgrim River Bridge}

One of the bridges that were selected was Pilgrim River Bridge. The Pilgrim river bridge is located in Houghton County, Michigan over Pilgrim River on US 41. Figure 63 shows the location of the selected bridge. Pilgrim River Bridge is a concrete arch bridge built in 1916. The span length of the bridge is about $17.75 \mathrm{~m}$ and the deck width is $12.19 \mathrm{~m}$. 


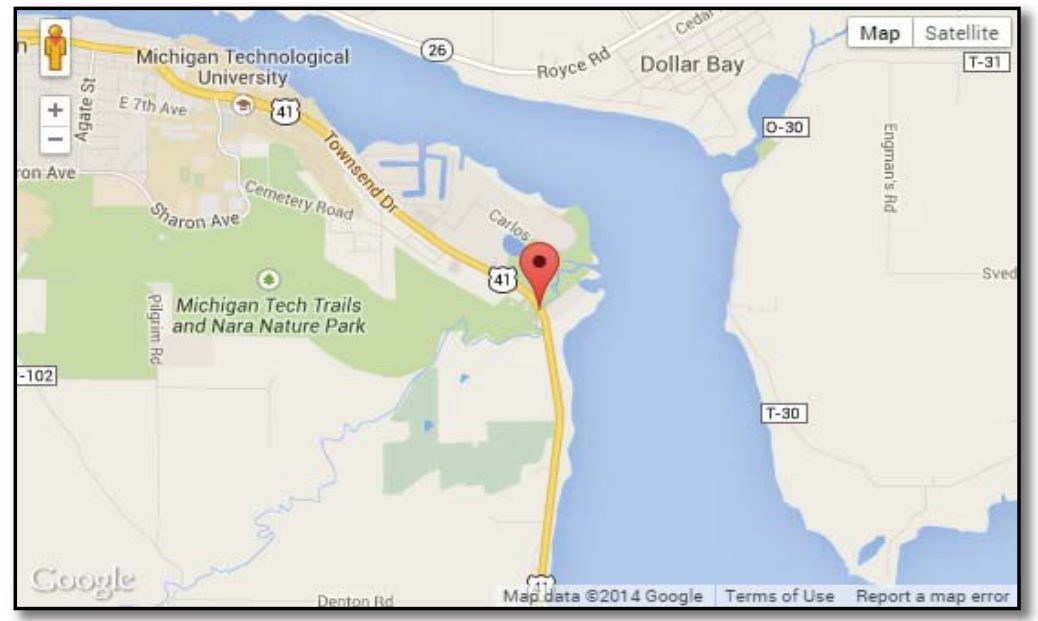

Figure 63. Pilgrim River Bridge (Image Source https://www.google.com/maps,See Appendix-A for Permissions)

\section{Sturgeon River Bridge}

The other bridge that was selected was the Sturgeon River Bridge. The Sturgeon river bridge is located 6 miles west of Baraga in Baraga County, Michigan over M 38. Figure 64 shows the location of the selected bridge.

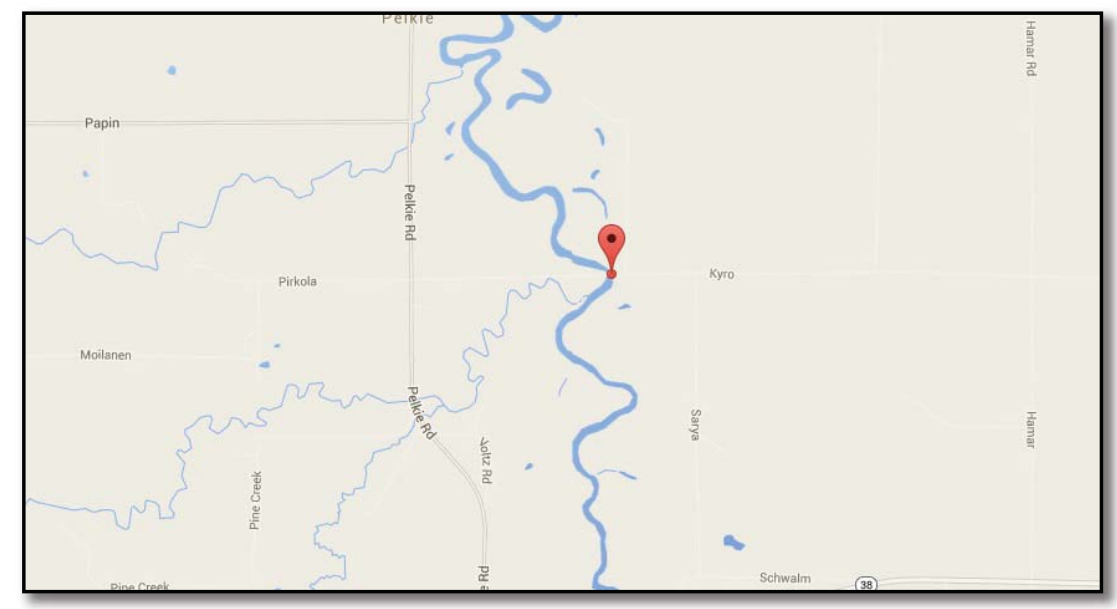

Figure 64. Sturgeon River Bridge (Image Source https://www.google.com/maps, See Appendix-A for Permissions) 


\section{Field Installation}

The main purpose of field deployment was to validate the installation of the proposed system. A detailed and careful study of the bridge plan and site investigation was made on US41 over Pilgrim River Bridge and M38 over Sturgeon River Bridge. The location where the posts and base station was to be installed was established and marked carefully on both the sites. To make the installation of scour sensor elements practical the previously described posts were deployed.

\section{Installation of the Monitoring System}

The bridge scour monitoring system was established and installed on US41 over Pilgrim River Bridge and on M38 over Sturgeon River Bridge. During the installation, several parties were involved. MDOT was responsible for the traffic control and the MDOT team provided the instruments required to install the posts and base station post.

Two posts were installed on each site for the purpose of validating the installation of the system. At the Pilgrim River site one post was installed on each bank at $4.49 \mathrm{~m}$ and $3.14 \mathrm{~m}$ from the bridge deck and at the Sturgeon River site the posts were installed $3.04 \mathrm{~m}$ and $4.57 \mathrm{~m}$ from the bridge deck. The base station post for both the sites was placed in the line of sight from the smart scour sensing posts at approximately $5.79 \mathrm{~m}$ and $22.55 \mathrm{~m}$ from the nearer post respectively.

An auger mounted on a crane was used to drill a hole in the river bank (Figure 65) and the posts were manually driven into the ground (Figure 66). The posts were then filled with small aggregates to make it stable and the soil around the posts was compacted.

The Base station assembly was mounted on a wooden post and hoisted in the air using a crane and carefully driven into the pre augured hole in the ground (Figure 67). The bottom of the post was compacted using cement to make it stable. The solar panel was mounted on top of the wooden post. An antenna to boost up the 
cellular link was added to the sturgeon river post due to poor cellular network signal at that location. The antenna was mounted on top of the wooden post using a flange assembly and oriented towards the nearest cell tower for better reception.

\section{Result of Field Deployment}

The field installation of the proposed system was practical. The smart scour-sensing posts made the field installation of the system easier. An auger mounted on a crane system that was used to drill holes for the posts were very effective and swift. The installation of the proposed system was validated during the field deployment task. However, the complete working of the system is yet to be verified in the field. 


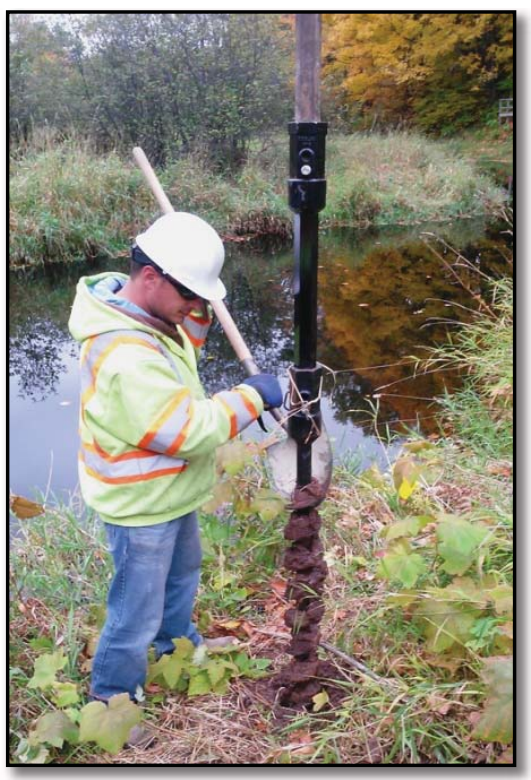

(a)

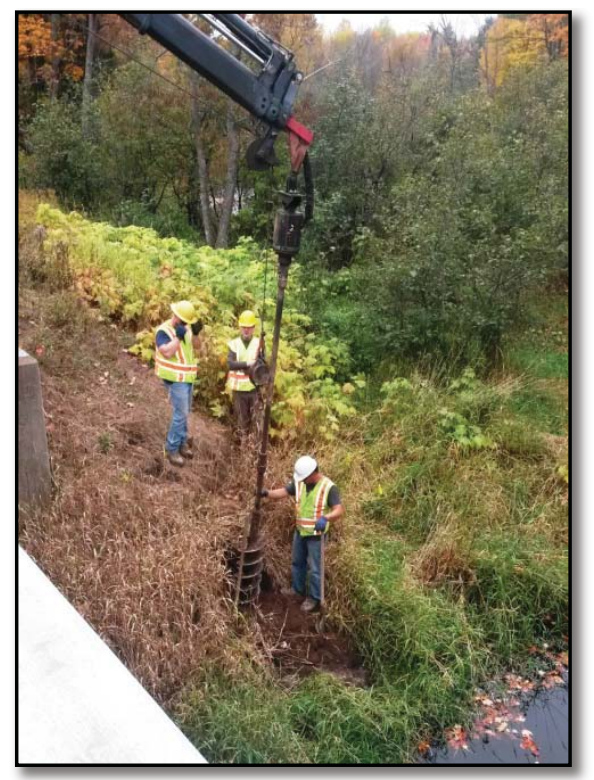

(b)

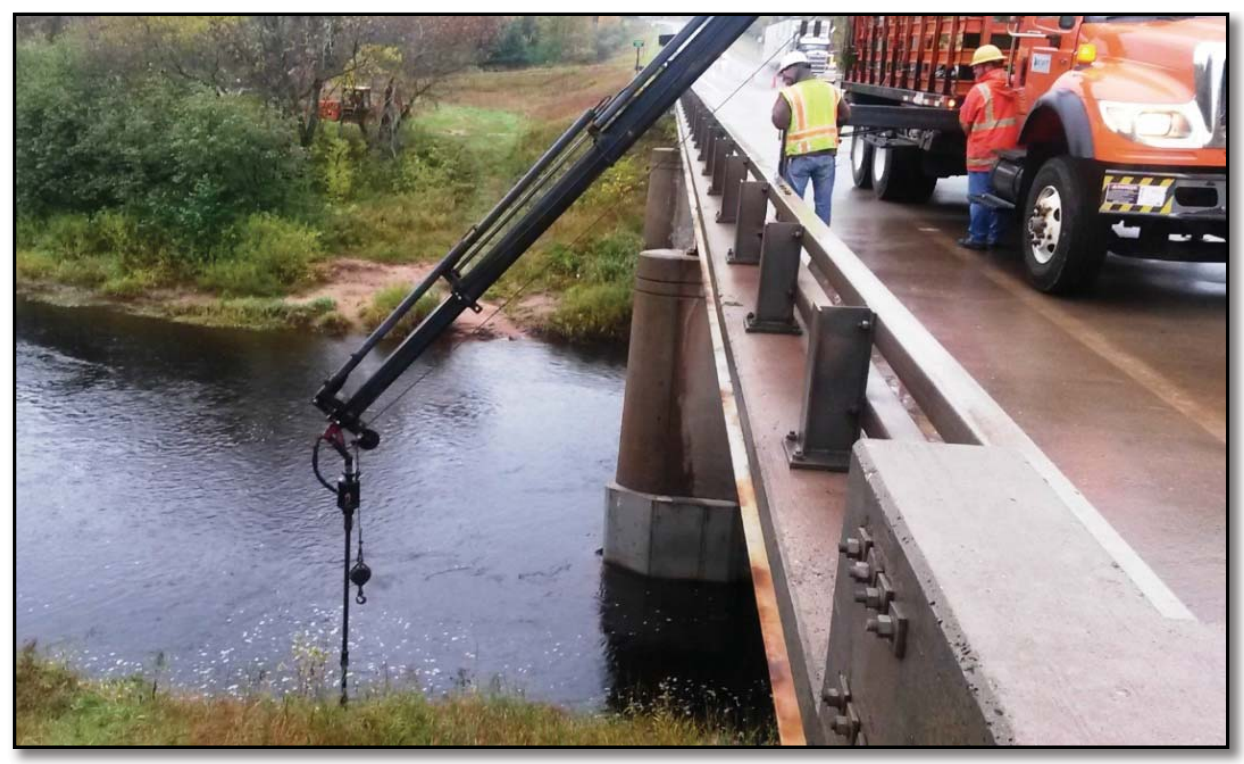

(c)

Figure 65. (a) and (b) Auger (c) Procedure for Drilling of Hole (Image by Author) 


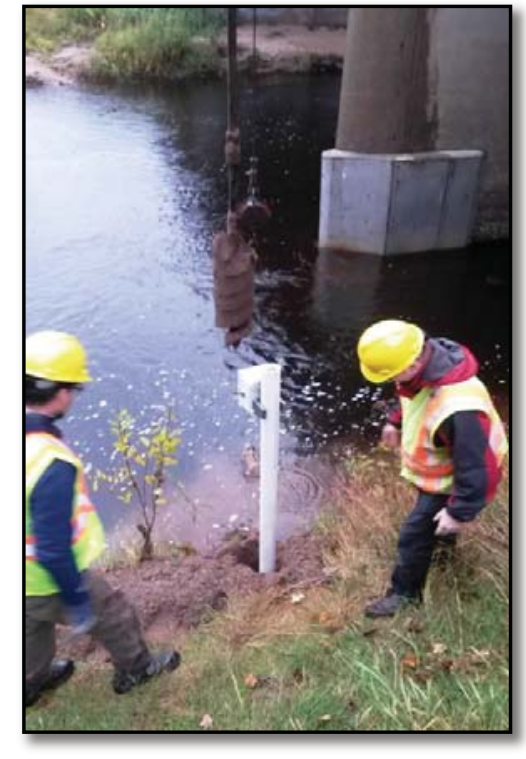

(a)

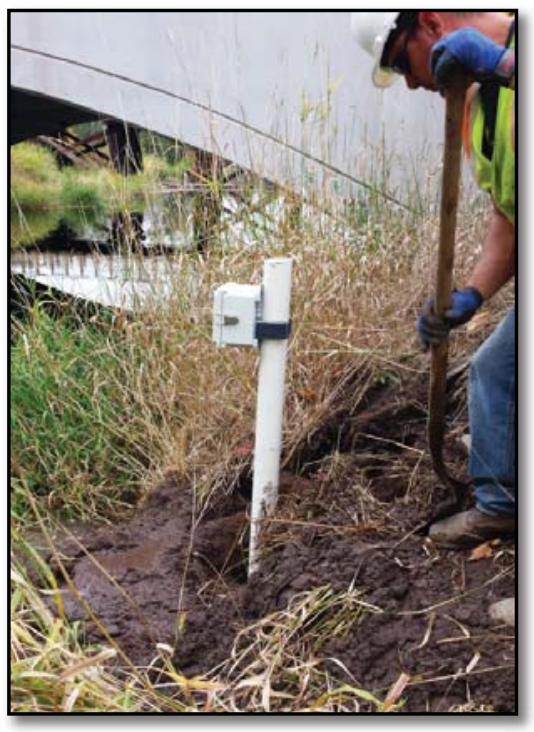

(c)

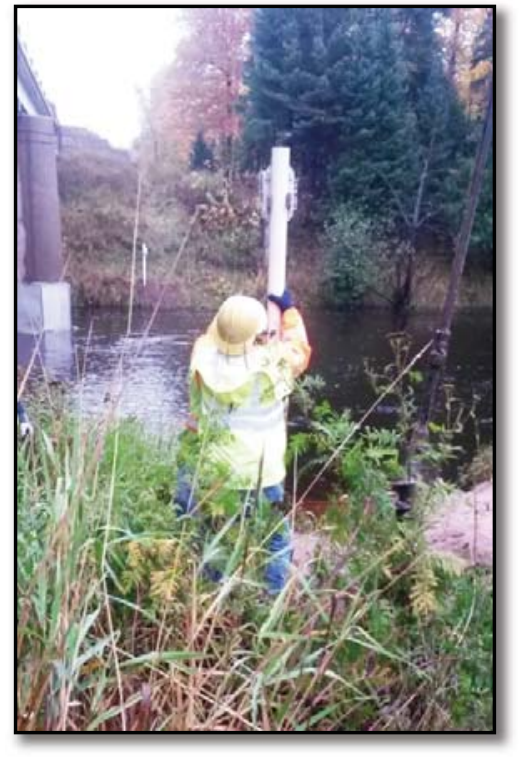

(b)

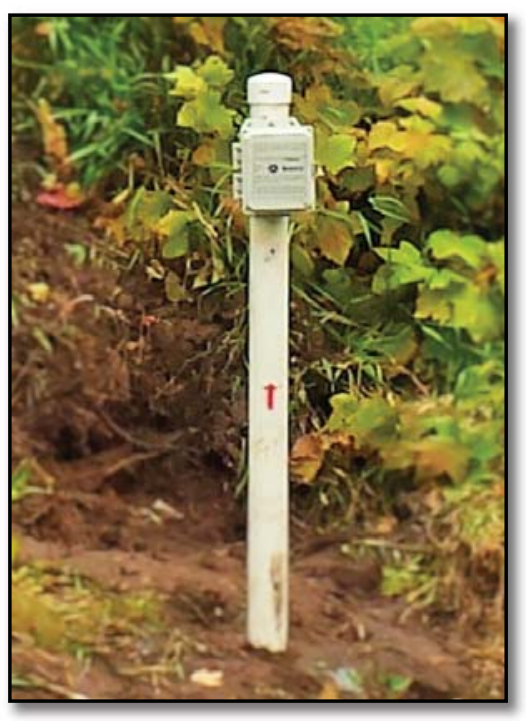

(d)

Figure 66. (a) and (b) Manual Installation of Post, (b) and (c) Installed post (Image by Author) 


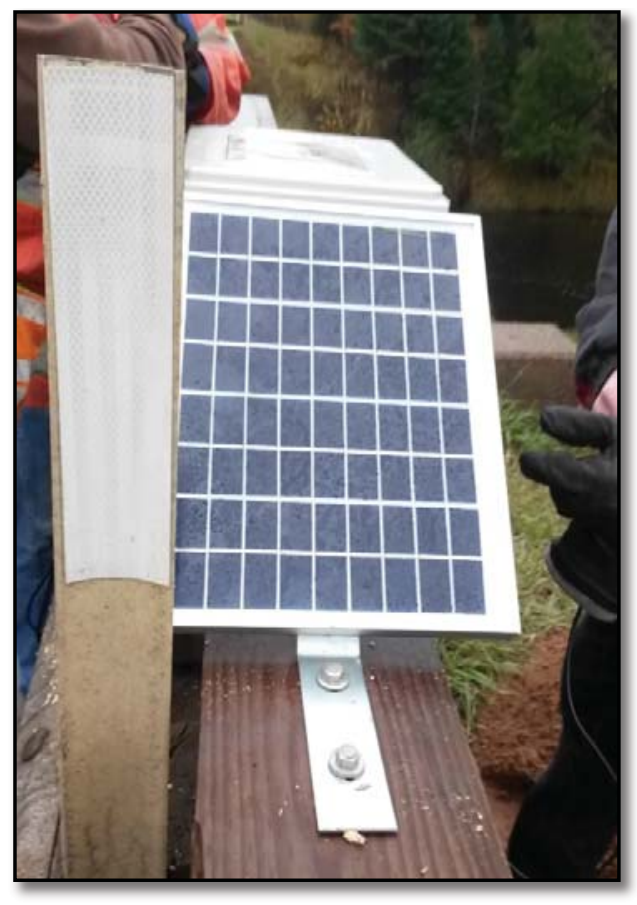

(a)

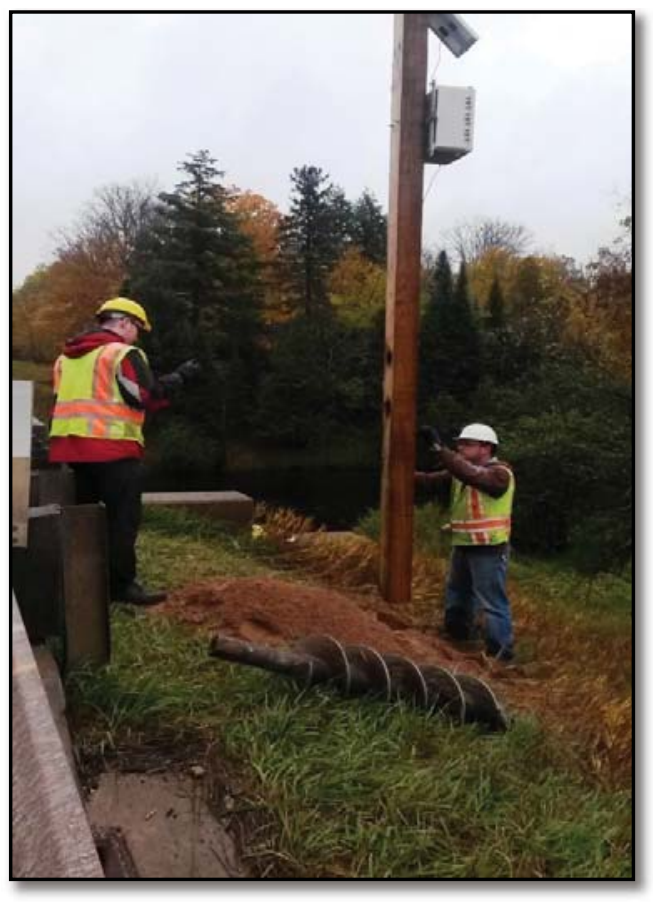

(b)

Figure 67. (a) Solar Panel and (b) Installation of Base Station (Image by Author) 


\section{Conclusion and Future Work}

We now summarize the thesis, discuss its findings and contributions, and point out limitations of the current work.

In this thesis an automated remote flow detection arrays based on bio-inspired flow sensors were introduced for scour monitoring. The method is simple because it uses robust magnetostrictive sensors and low power Narada wireless sensors for data acquisition. The method takes advantage of a simple algorithm and pre-known depth and state of the sensors to determine the extent of scour.

The proof-of-concept experiments were performed in the laboratory to validate the ability of an automated scour detection and monitoring system to successfully monitor scour. The laboratory results demonstrated that distinction between static and dynamic sensor signals can be made, and, with the knowledge of the depth of the sensor, scour can be measured and monitored. The laboratory tests established the automated characteristics of the system and displayed the ability of the system to successfully give a warning of impending bridge failure. The ability of the system under study to automatically capture and $\log$ peak scour events was shown. Furthermore, the algorithm used in this system is simple which aids its autonomy and the results that are relayed to the bridge owner are easy to comprehend.

The hardware components that make the system were discussed. It was evident that all the components involved in the system are relatively easily available and are inexpensive. This thesis also presented the simplicity of the procedure that was adopted for field installation which makes the system easy to handle and install and suitable for mass installation.

However, the system is yet to be tested in the field. The conditions encountered in real life can be very different compared to the laboratory experiments due to the countless numbers of random processes in the environment. The systems robustness and the algorithm used must be validated with field tests for completeness. 
Based on the success of the laboratory trial tests, a comprehensive scour monitoring system was designed for implementation on the field for testing purpose.

\section{Future Work}

While this thesis has demonstrated the ability and the effectiveness of the proposed scour monitoring system to monitor scour successfully with the presented laboratory results, the system could be further developed in a number of ways. The next section presents some of these directions.

\section{Extended Field Testing Campaigns}

The scour monitoring system developed has recently been installed in the field at the selected bridge site for field testing. The performance of the system in the field is yet to be analyzed. The future research should focus on testing the scour monitoring system in the field to test the long-term performance of the system.

\section{Improve Robustness of Transducers}

Three different versions of transducers were presented in this thesis, each a better from of the previous. Additional laboratory and field work is still necessary to improve on the robustness of the transducers. The tests conducted were clear water tests; therefore, to properly characterize the robustness, tests should be performed in water with high sediments and debris to validate the ability of the sensors to survive in difficult conditions. Among the practical issues that require further considerations is the impact of debris and tree leaves on the behavior and robustness of the sensors. 


\section{Room for Improving Algorithm and Threshold}

To make the presented computational framework suitable for practical application, future research should also focus on developing efficient algorithms and improving the sensitivity and precision of the automated data interrogation algorithms to lowflow conditions. The thresholds for flow conditions can be more accurately set to improve the efficiency of the system. 


\section{Acknowledgements and Disclaimer}

This work is supported by the Commercial Remote Sensing and Spatial Information Technologies program of the U.S. Department of Transportation (USDOT) Office of the Assistant Secretary for Research and Technology, Cooperative Agreement \#RITARS-12-H-MTU, with additional support provided by the Michigan Department of Transportation (MDOT), the Maryland State Highway Administration (MDSHA), Michigan Technological University, the Michigan Tech Research Institute, Civionics, and the Center for Automotive Research.

The views, opinions, findings, and conclusions reflected in this presentation are the responsibility of the authors only and do not represent the official policy or position of the USDOT/OST-R, MDOT, MDSHA, or any other entity. 


\section{References}

[1] P. Lagasse, P. Clopper, J. Pagan-Ortiz et al., Bridge Scour and Stream Instability Countermeasures: Experience, Selection and Design Guidance. Volume 2, 2009.

[2] E. Richardson, and S. Davis, "Evaluating Scour at Bridges . Federal Highway Administration, Hydraulic Engineering Circular No. 18," Publication FHWA NHI, pp. 01-001, 2001.

[3] L. Prendergast, and K. Gavin, "A review of bridge scour monitoring techniques," Journal of Rock Mechanics and Geotechnical Engineering, vol. 6, no. 2, pp. 138-149, 2014.

[4] K. G. Luke J. Prendergasta, "Monitoring of scour critical bridges using changes in the natural frequency of vibration of foundation piles - A field investigation."

[5] P. E. Beatrice E. Hunt, M.ASCE1, Senior Hydraulics Engineer, "ESTABLISHING A SCOUR MONITORING."

[6] B. E. Hunt, Monitoring scour critical bridges: Transportation Research Board, 2009.

[7] D. V. Swenson, and A. R. Ingraffea, "The collapse of the Schoharie Creek Bridge: a case study in concrete fracture mechanics," International journal of fracture, vol. 51, no. 1, pp. 73-92, Sep 1, 1991.

[8] D. M. Frangopol, A. Strauss, and S. Kim, "Bridge reliability assessment based on monitoring," Journal of Bridge Engineering, vol. 13, no. 3, pp. 258-270, May-Jun, 2008.

[9] R. T. a. I. Friedland1, ""Status of Scour Instrumentation Development"."

[10] W. Zheng, Instrumentation and Computational Modeling for Evaluation of Bridge Substructures across Waterways , Final Report for State Study-229, FHWA/MS-DOT-RD-13-229, MS-DOT-RD-13-229, December 2013.

[11] P. Lagasse, E. Richardson, and J. Schall, "Fixed instrumentation for monitoring scour at bridges," Transportation Research Record: Journal of the Transportation Research Board, vol. 1647, no. 1, pp. 1-9, 1998.

[12] J. Lefter, "Instrumentation for measuring scour at bridge piers and abutments," NCHRP Research Results Digest, no. 189, 1993.

[13] N. Yankielun, and L. Zabilansky, "Laboratory investigation of time-domain reflectometry system for monitoring bridge scour," Journal of Hydraulic engineering, vol. 125, no. 12, pp. 1279-1284, Dec, 1999.

[14] R. Ettema, T. Nakato, and M. V.-I. Muste, An illustrated guide for monitoring and protecting bridge waterways against scour, IIHRHydroscience \& Engineering, University of Iowa, 2006. 
[15] A. Zarafshan1, A. Iranmanesh2, and M. A. and Farhad Ansari, "VibrationBased Method and Sensor for Monitoring of Bridge Scour," Journal of Bridge Engineering,ASCE, November 6, 2012.

[16] D. S. Mueller, J. D. Abad, C. M. García et al., "Errors in acoustic Doppler profiler velocity measurements caused by flow disturbance," Journal of hydraulic Engineering, vol. 133, no. 12, pp. 1411-1420, Dec, 2007.

[17] G. Placzek, and F. Haeni, Surface-geophysical techniques used to detect existing and infilled scour holes near bridge piers: US Department of the Interior, US Geological Survey, 1995.

[18] P. Downey, and A. Flatau, "Magnetoelastic bending of Galfenol for sensor applications," Journal of Applied Physics, vol. 97, no. 10, pp. 10R505, May $15,2005$.

[19] V. M. Karbhari, and F. Ansari, Structural health monitoring of civil infrastructure systems: Elsevier, 2009.

[20] J. Kim, A. Swartz, J. P. Lynch et al., "Rapid-to-deploy reconfigurable wireless structural monitoring systems using extended-range wireless sensors," Smart Structures and Systems, vol. 6, no. 5-6, pp. 505-524, JulAug, 2010.

[21] "Civionics Custom Wireless Sensing and Control Solution," http://civionics.com/wp/products/narada/.

[22] "Winsystems," http://www.winsystems.com/PPM-LX800-G.CFM.

[23] "Verizon," http://www.verizonwireless.com/support/4g-lte-global-usbmodem-uml290/.

[24] R. A. Swartz, D. Jung, J. P. Lynch et al., "Design of a wireless sensor for scalable distributed in-network computation in a structural health monitoring system." pp. 1570-1577.

[25] P. Korpipää, M. Koskinen, J. Peltola et al., "Bayesian approach to sensorbased context awareness," Personal and Ubiquitous Computing, vol. 7, no. 2, pp. 113-124, 2003.

[26] A. Zwartjes, P. Havinga, G. Smit et al., "Using Unsupervised Learning to Improve the Naive Bayes Classifier for Wireless Sensor Networks." pp. 7176. 


\section{Appendix-A}

Figure 3 and Figure 12

Material in the public domain does not need permission.

Figure 36

From Microsoft (C) use of Microsoft Copyrighted Content http://www.microsoft.com/en-us/legal/intellectualproperty/permissions/

You may use images or content from Microsoft products and services in school reports provided that you comply with the guidelines above. You may critique or comment on the product or service.

You may not use screenshots of Microsoft product boot-up screens, opening screens, "splash screens," or screens from beta release products or other products that have not been commercially released. You may use other screenshots in advertising, in documentation (including educational brochures), in tutorial books, in videos, or on websites, provided that, in addition to the requirements above, you:

- Do not alter the screenshot except to resize it.

- Do not use portions of screenshots.

- Do not include screenshots in your product user interface.

- Do not use screenshots that contain third-party content.

- Do not use screenshots that contain an image of an identifiable individual. 
Figure 63 and Figure 64

From Google (C) Permission Guidelines for Google Maps and Google Earth

Google. 2014. Permissions. www.google.com/permissions. Web.

"Google Maps and Earth has built-in print functionality. You may print Content

from Maps and Earth for personal use and enlarge it. In all uses where print will be distributed, first be sure to read our FAQ on applicable product Terms of Service and fair use. Second, all uses must properly show attribution to both Google and our data providers. Please see our attribution page for more information.

When using Google Maps and Earth Content in print, any images used must reflect how they would look on online. For example, you are not allowed to make any changes (e.g. delete, blur, etc.) to our products that would make them look genuinely different. This includes, but is not limited to, adding clouds or other natural elements, altered user-interfaces, and modification that do not appear in the actual product. No explicit permission is required for your print project. We are unable to sign any letter or contract specifying that your project has our explicit permission."

"You can find the attribution in the line(s) shown on the bottom of the Content in the products along with copyright notices, such as "Map data (C2012 Google, Sanborn". Note that the exact text of the attribution changes based on geography and content type.

The attribution text must be legible to the average viewer or reader.' 